\title{
Synthesis, Antitumor and Antimicrobial Activity of Novel 1-Substituted Phenyl-3-[3-alkylamino(methyl)-2-thioxo-1,3,4-oxadiazol-5-yl] $\beta$-Carboline Derivatives
}

\author{
Franciele C. Savariz, ${ }^{a}$ Anelise S. N. Formagio, ${ }^{a}$ Valéria A. Barbosa, ${ }^{a}$ \\ Mary Ann Foglio, ${ }^{b}$ João E. de Carvalho, ${ }^{b}$ Marta C. T. Duarte, ${ }^{b}$ \\ Benedito P. Dias Filho and Maria Helena Sarragiotto*,a
}

\author{
${ }^{a}$ Departamento de Química and ${ }^{c}$ Departamento de Análises Clínicas, \\ Universidade Estadual de Maringá, 87020-900 Maringá-PR, Brazil \\ ${ }^{b}$ Centro Pluridisciplinar de Pesquisas Químicas, Biológicas e Agrícolas, \\ Universidade Estadual de Campinas, CP 6171, 13083-970 Campinas-SP, Brazil
}

\begin{abstract}
Com o propósito de aumentar a atividade anticâncer demonstrada anteriormente pelas 1-fenilssubstituído-3-(2-tioxo-1,3,4-oxadiazol-5-il) $\beta$-carbolinas 1a-c, neste trabalho foram realizadas a síntese e a avaliação in vitro da atividade antitumoral de novas bases de Mannich 2-7(a-c), derivadas da introdução de diferentes grupos alquilamino(metil) na unidade 1,3,4-oxadiazol de 1a-c. Os derivados 1a-c e 2-7(a-c) foram também avaliados quanto às atividades antibacteriana e antifúngica. Adicionalmente, um estudo in silico das propriedades de ADME dos novos compostos sintetizados 2-7(a-c) foi realizado pela avaliação de seus parâmetros de Lipinski e de dados de área de superfície topológica polar (TPSA) e de porcentagem de absorção (\% ABS).
\end{abstract}

With the purpose of activity enhancement of 1-substituted phenyl-3-(2-thioxo-1,3,4-oxadiazol5 -yl) $\beta$-carbolines 1a-c, reported as potential antitumor agents in our previous study, herein we report the synthesis and antitumor activity evaluation of several novel Mannich bases 2-7(a-c), by the introduction of different alkylamino(methyl) groups in the 1,3,4-oxadiazole unity of 1a-c. The antimicrobial activities of 1a-c and of 2-7(a-c) were also evaluated. Additionally, an in silico study of the ADME properties of novel synthesized $\beta$-carboline derivatives 2-7(a-c) was performed by evaluation of their Lipinski's parameters and topological polar surface area (TPSA) and percentage of absorption (\% ABS) data.

Keywords: 1,3-disubstituted $\beta$-carboline derivatives, 1,3,4-oxadiazole, Mannich bases, antitumor activity, antimicrobial activity

\section{Introduction}

$\beta$-Carboline alkaloids are a class of synthetic and naturally occurring compounds that possess a large spectrum of important pharmacological properties. ${ }^{1}$ Particularly, these compounds have shown antitumor activity, ${ }^{1-10}$ acting mainly as intercalating into DNA ${ }^{6-10}$ Also, synthetic and natural compounds of this class exhibited antimicrobial activities, mainly against Bacillus subtilis, Escherichia coli and Staphylococcus aureus bacteria and against Candida albicans fungi. ${ }^{11-13}$

Studies on a variety of synthetic $\beta$-carboline derivatives have demonstrated the influence of both molecule planarity

*e-mail: mhsarragiotto@uem.br and substituents nature in C-1, C-3 and position -9 of the $\beta$-carboline skeleton on activity. ${ }^{4,5}$ The presence of appropriate substituents on these positions could lead to more potent compounds with reduced toxicity.

The biological potential of $\beta$-carboline alkaloids and the importance of the search for new antitumor and antimicrobial agents have led us to study this class of compounds. In previous work, we reported synthesis and in vitro antitumor activities of a series of 1-substituted phenyl $\beta$-carboline bearing 2-thioxo-1,3,4-oxadiazol-5-yl moiety at C-3. ${ }^{14}$ The anticancer assay results pointed compounds 1a-c with growth inhibition effect $\left(\mathrm{GI}_{50}<100 \mu \mathrm{mol} \mathrm{L}^{-1}\right)$ for all eight human cancer cell lines tested. ${ }^{14}$

Taking in account our previous results and with the aim of increasing the antitumor activity we have continued our 
work on $\beta$-carboline derivatives $\mathbf{1}$, with the introduction of substituents in 1,3,4-oxadiazole ring. Literature reports that Mannich bases of oxadiazoles possess important activities, such as antibacterial and anticancer. ${ }^{15-17}$

Therefore, in this study we synthesized six series of Mannich bases from the previously reported active compounds 1a-c ${ }^{14}$ to investigate the effects of different 3-alkylamino(methyl) substituents on the oxadiazole ring, expecting that the incorporation of these substituents would lead to antitumor activity enhancement. More specifically, we report the synthesis and in vitro anticancer activity of $\beta$-carboline derivatives 2-7(a-c) bearing a 3-alkylamino(methyl)-2-thioxo-1,3,4-oxadiazol-5-yl group at C-3. Additionally, the antimicrobial activities of $\mathbf{1 a - c}$ and of the Mannich bases 2-7(a-c) were evaluated.

An in silico study of the ADME properties of novel synthesized $\beta$-carboline derivatives was carried out by investigation of their Lipinski's parameters, topological polar surface area (TPSA) and percentage of absorption (\% ABS) ${ }^{18,19}$

\section{Results and Discussion}

\section{Chemistry}

The 3-(2-thioxo-1,3,4-oxadiazol-5-yl) $\beta$-carbolines 1a-c were prepared from the commercial $L$-tryptophan as previously reported. ${ }^{14}$ The condensation reaction of 1a-c, formaldehyde $37 \%^{15,17}$ and the primary amines isopropylamine, butylamine, cyclohexylamine and benzylamine, using ethanol as solvent, afforded the Mannich bases 2a-c, 3a-c, 4a-c and 5a-c, respectively (Scheme 1). Condensation reaction with pyrrolidine and morpholine secondary amines under the same conditions, afforded the Mannich bases 6a-c and 7a-c, respectively (Scheme 1). The Mannich bases 2-7(a-c) were obtained in range of 52\% to $92 \%$ yields from 1a-c, and in six steps from commercial $L$-tryptophan with $15-34 \%$ overall yields.

All novel compounds were characterized by their spectroscopic data (IR, EIMS, ${ }^{1} \mathrm{H}$ and ${ }^{13} \mathrm{C}$ NMR), which are described in the Experimental section.

The formation of Mannich bases 2-7(a-c) was evidenced by the presence of a singlet at $\delta_{\mathrm{H}} 5.00-6.00$ in the ${ }^{1} \mathrm{H}$ NMR spectra, corresponding to the methylene exocyclic hydrogens of the alkylamino(methyl) group introduced at $N-3$ of the 1,3,4-oxadiazole ring, together with the signals for each group alkylamino. The exocyclic methylene carbon of the alkylamino group appears in the region of $\delta_{C} 60-70$ in the ${ }^{13} \mathrm{C}$ NMR. The signals in the $\delta_{\mathrm{C}} 177$ and 160 region were assigned for $\mathrm{C}-2$ and $\mathrm{C}-5$ carbons, respectively, of the 1,3,4-oxadiazole ring.

The IR spectra showed absorption bands characteristic for $\mathrm{N}-\mathrm{H}$ indole and $\mathrm{C}=\mathrm{N}$ stretching in the 3040 and $1615 \mathrm{~cm}^{-1}$ region, respectively, and for $\mathrm{C}=\mathrm{S}$ group at 1390 and $1240 \mathrm{~cm}^{-1}$.

The structures of 2-7(a-c) were also confirmed by EI mass spectra. The majority of compounds showed the presence of a base peak at $\mathrm{m} / z$ [M+-alkylamino(methyl)-<smiles>[R]Cc1cccc(-c2nc(-c3n[nH]c(=S)o3)cc3c2-c2[nH]c4ccccc4c2-3)c1</smiles>

1a-c

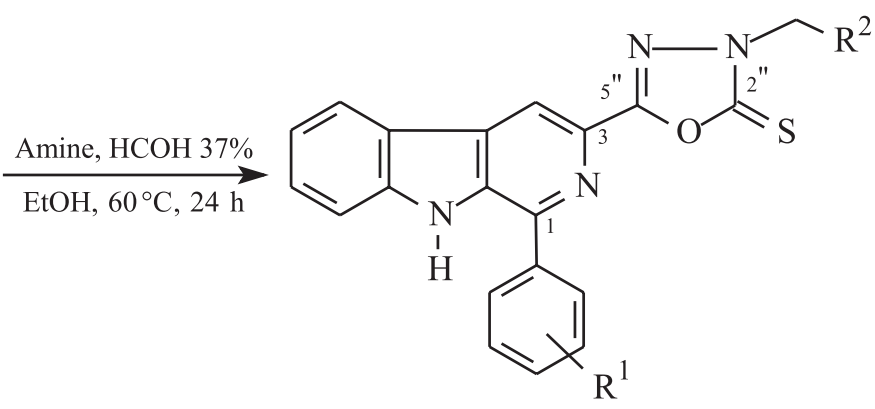

2-7 (a-c)
a: $\mathrm{R}^{1}=\mathrm{H}$
b: $\mathrm{R}^{1}=m-\mathrm{NO}_{2}$
c: $\mathrm{R}^{1}=p-\mathrm{N}\left(\mathrm{CH}_{3}\right)_{2}$

2: $\mathrm{R}^{2}=-\mathrm{NH}-$

3: $\mathrm{R}^{2}=-\mathrm{NH} \bigcirc$<smiles></smiles>

$4: \mathrm{R}^{2}=-\mathrm{NH} \longrightarrow$<smiles>[R]CN1CCOCC1</smiles> 
thioxo-1,3,4-oxadiazole] relative to cleavage between $\mathrm{C}-3$ of $\beta$-carboline and C-5 of 1,3,4-oxadiazole ring. For the benzyl derivatives 5a-c the base peaks were observed at $m / z 91$, which was due to the tropilium ion formation.

\section{Anticancer activity}

The $\mathrm{GI}_{50}$, TGI and $\mathrm{LC}_{50}$ data and MG-MID values obtained for each compound and cell lines tested were summarized in Tables 1 and 2. The anticancer assay results pointed towards eleven compounds (2a, 2c, 3a, 5b, 5c, 6a-c and 7a-c) with growth inhibition effect $\left(\mathrm{GI}_{50}<100 \mu \mathrm{mol} \mathrm{L}^{-1}\right)$ for all eight human cancer cell lines tested, showing $\mathrm{GI}_{50}$ (MG-MID) values within 4.37 to $36.31 \mu \mathrm{mol} \mathrm{L} \mathrm{L}^{-1}$ range (Table 1). Analysis of the TGI (MG-MID) values (Table 2) showed that the Mannich bases synthesized were able to inhibit total growth (cytostatic activity), except for $\mathbf{3 b}$ and 7c, in a concentration minor than $100 \mu \mathrm{mol} \mathrm{L} \mathrm{L}^{-1}$.

Among compounds tested, $\mathbf{2 a}, \mathbf{2} \mathbf{c}$ and $\mathbf{5 c}$ were the most active derivatives, exhibiting a broad antitumor activity spectrum at $\mathrm{GI}_{50}$ and TGI levels, with $\mathrm{GI}_{50}$ (MGMID) values of 5.89, 4.37 and $4.57 \mu \mathrm{mol} \mathrm{L}^{-1}$ (Table 1), respectively. Moreover, compound 2a displayed cytotoxic efficacy with $\mathrm{LC}_{50}$ (MG-MID) value of $60.49 \mu \mathrm{mol} \mathrm{L}^{-1}$ (Table 2).

A potent activity against melanoma (UACC-62) and lung (NCI-460) cell lines, with $\mathrm{GI}_{50}$ values of 0.88 and $1.01 \mu \mathrm{mol} \mathrm{L} \mathrm{L}^{-1}$ (Table 1), respectively, was observed for compound 2c, which possess the N,N-dimethylphenyl and isopropylamino(methyl) groups at C-1 and C-3, respectively, of the $\beta$-carboline nucleus. Compound $\mathbf{5 c}$ also demonstrated a significant activity, with $\mathrm{GI}_{50}$ values in the range of 0.38 and $2.98 \mu \mathrm{mol} \mathrm{L^{-1 }}$ towards four cell lines (melanoma, ovarian resistant, renal and lung).

Comparison of the $\mathrm{GI}_{50}$ (MG-MID) values for all tested compounds showed that, except for $\mathbf{3 b}, \mathbf{4 c}$ and $\mathbf{7 c}$, the Mannich bases derivatives displayed higher anticancer activity than the corresponding precursors. For the most active 2a, 2c and 5c Mannich bases, the introduction of an alkylamino(methyl) group at N-3 of the heterocyclic ring

Table 1. $\mathrm{GI}_{50}$ values (in $\mu \mathrm{mol} \mathrm{L} \mathrm{L}^{-1}$ ) and $\mathrm{GI}_{50} \mathrm{MG}-\mathrm{MID}$ (in $\mu \mathrm{mol} \mathrm{L} \mathrm{L}^{-1}$ ) for compounds 1a-c and 2-7(a-c)

\begin{tabular}{|c|c|c|c|c|c|c|c|c|c|}
\hline \multirow[t]{2}{*}{ Compounds } & \multicolumn{9}{|c|}{ Cancer cell line } \\
\hline & $\begin{array}{c}\text { Melanoma } \\
\text { UACC-62 }\end{array}$ & $\begin{array}{l}\text { Breast } \\
\text { MCF7 }\end{array}$ & $\begin{array}{c}\text { Ovarian } \\
\text { resistant } \\
\text { NCI/ADR }\end{array}$ & $\begin{array}{l}\text { Renal } \\
786-0\end{array}$ & $\begin{array}{c}\text { Lung } \\
\text { NCI-460 }\end{array}$ & $\begin{array}{l}\text { Prostate } \\
\text { PCO-3 }\end{array}$ & $\begin{array}{l}\text { Ovarian } \\
\text { OVCAR }\end{array}$ & $\begin{array}{l}\text { Colon } \\
\text { HT29 }\end{array}$ & MG-MID $^{a}$ \\
\hline Doxorubicin & 0.43 & 0.005 & 0.13 & 0.21 & 0.04 & 0.46 & 0.34 & 0.46 & 0.14 \\
\hline 1a & $>100$ & 25.54 & 17.60 & 27.95 & 20.60 & 25.54 & 31.77 & 25.54 & 29.25 \\
\hline $1 b$ & 24.10 & 27.93 & 74.99 & 32.38 & 73.41 & 12.20 & 22.31 & 27.93 & 31.26 \\
\hline 1c & 23.82 & 18.84 & 32.11 & 14.72 & 19.09 & 14.72 & 14.72 & 40.46 & 20.83 \\
\hline $2 a$ & 6.00 & 0.86 & 5.90 & 4.66 & 5.72 & 6.12 & 6.21 & 43.85 & 5.89 \\
\hline $2 b$ & 7.08 & 4.56 & 9.27 & 49.90 & 4.26 & 53.54 & 6.25 & $>100$ & 14.79 \\
\hline $2 \mathrm{c}$ & 0.88 & 5.88 & 2.78 & 9.82 & 1.01 & 13.31 & 3.42 & 22.06 & 4.37 \\
\hline $3 \mathbf{a}$ & 5.29 & 7.29 & 30.16 & 6.51 & 2.74 & 26.66 & 5.99 & 49.80 & 10.72 \\
\hline $3 b$ & $>100$ & $>100$ & $>100$ & $>100$ & $>100$ & $>100$ & $>100$ & $>100$ & 100.00 \\
\hline $3 c$ & 10.34 & 42.04 & 16.32 & 5.03 & 3.99 & 35.24 & 39.05 & $>100$ & 19.50 \\
\hline $4 a$ & 8.89 & 40.79 & 13.49 & 28.57 & 7.02 & 38.99 & 11.21 & $>100$ & 21.38 \\
\hline $4 b$ & 7.58 & 14.33 & 3.74 & 49.81 & 4.33 & 20.31 & 12.28 & $>100$ & 14.79 \\
\hline $4 c$ & 8.74 & $>100$ & 5.13 & 52.44 & 15.19 & 58.48 & 18.66 & $>100$ & 28.18 \\
\hline $5 \mathbf{a}$ & $>100$ & 16.87 & 3.72 & 1.31 & 4.63 & 47.68 & 6.39 & 54.29 & 12.59 \\
\hline $5 b$ & 11.30 & 16.64 & 5.34 & 7.13 & 5.25 & 14.17 & 5.55 & 68.52 & 10.96 \\
\hline $5 c$ & 2.98 & 10.23 & 2.55 & 0.38 & 1.28 & 14.96 & 5.91 & 56.34 & 4.57 \\
\hline $6 a$ & 7.61 & 22.81 & 6.83 & 9.45 & 7.58 & 22.64 & 6.97 & 21.53 & 11.48 \\
\hline $6 b$ & 6.97 & 11.23 & 6.65 & 15.98 & 17.98 & 14.25 & 6.51 & 24.15 & 11.75 \\
\hline $6 c$ & 6.63 & 16.08 & 7.05 & 21.81 & 11.04 & 11.00 & 5.96 & 14.13 & 10.72 \\
\hline $7 \mathbf{a}$ & 6.77 & 39.43 & 7.59 & 11.42 & 36.77 & 8.10 & 7.38 & 17.61 & 13.18 \\
\hline $7 b$ & 6.81 & 11.49 & 7.96 & 51.73 & 40.43 & 6.33 & 10.38 & 38.91 & 15.49 \\
\hline $7 c$ & 24.19 & 56.35 & 20.16 & 67.63 & 38.55 & 16.58 & 47.33 & 49.28 & 36.31 \\
\hline
\end{tabular}

${ }^{\mathrm{a}} \mathrm{GI}_{50}$ mean-graph midpoint (MG-MID) = average sensitivity of all cell lines toward the test compounds. 
Table 2. TGI (in $\mu \mathrm{mol} \mathrm{L} \mathrm{L}^{-1}$ ) and $\mathrm{LC}_{50}$ (in $\mu \mathrm{mol} \mathrm{L} \mathrm{L}^{-1}$, values in parentheses) and TGI and $\mathrm{LC}_{50}$ MG-MID ( $\mu \mathrm{mol} \mathrm{L} \mathrm{L}^{-1}$ ) for compounds 1a-c and 2-7(a-c)

\begin{tabular}{|c|c|c|c|c|c|c|c|c|c|}
\hline \multirow[t]{2}{*}{ Compounds } & \multicolumn{9}{|c|}{ Cancer cell line } \\
\hline & $\begin{array}{l}\text { Melanoma } \\
\text { UACC-62 }\end{array}$ & $\begin{array}{l}\text { Breast } \\
\text { MCF7 }\end{array}$ & $\begin{array}{c}\text { Ovarian } \\
\text { resistant } \\
\mathrm{NCI} / \mathrm{ADR}\end{array}$ & $\begin{array}{l}\text { Renal } \\
786-0\end{array}$ & $\begin{array}{c}\text { Lung } \\
\text { NCI } 460\end{array}$ & $\begin{array}{c}\text { Prostate } \\
\text { PCO-3 }\end{array}$ & $\begin{array}{l}\text { Ovarian } \\
\text { OVCAR }\end{array}$ & $\begin{array}{l}\text { Colon } \\
\text { HT29 }\end{array}$ & MG-MID \\
\hline Doxorubicin & $0.44(0.99)$ & 0.09 (2.78) & $6.14(68.44)$ & $10.00(>100)$ & $1.25(>100)$ & $4.59(82.12)$ & $2.61(>100)$ & $10.58(>100)$ & $2.09(33.37)$ \\
\hline 1a & $>100$ & $>100$ & $>100$ & $>100$ & $>100$ & $>100$ & $>100$ & $>100$ & $>100(>100)$ \\
\hline $1 b$ & $>100$ & $>100$ & $>100$ & $>100$ & $>100$ & $35.77(>100)$ & $>100$ & $>100$ & $87.1(>100)$ \\
\hline 1c & $>100$ & $>100$ & $>100$ & $>100$ & $>100$ & $>100$ & $>100$ & $>100$ & $>100(>100)$ \\
\hline $2 a$ & $15.63(56.88)$ & $16.01(74.61)$ & $20.10(63.05)$ & $3.96(20.62)$ & $17.63(61.95)$ & $30.91(>100)$ & $14.64(52.41)$ & $81.39(>100)$ & $18.36(60.49)$ \\
\hline $2 b$ & $>100$ & $98.35(>100)$ & $>100$ & $>100$ & $88.90(>100)$ & $>100$ & $67.50(>100)$ & $>100$ & $93.62(>100)$ \\
\hline $2 c$ & $6.81(>100)$ & $>100$ & 39.16 & $>100$ & $>100$ & $>100$ & $95.97(>100)$ & $>100$ & $63.25(>100)$ \\
\hline $3 \mathbf{a}$ & $15.12(58.99)$ & $>100$ & $>100$ & $43.71(>100)$ & $>100$ & $>100$ & $25.59(>100)$ & $>100$ & $60.05(93.62)$ \\
\hline $3 \mathbf{b}$ & $>100$ & $>100$ & $>100$ & $>100$ & $>100$ & $>100$ & $>100$ & $>100$ & $>100(>100)$ \\
\hline $3 \mathrm{c}$ & $57.49(>100)$ & $>100$ & $>100$ & $21.55(>100)$ & $>100$ & $>100$ & $>100$ & $>100$ & $77.03(>100)$ \\
\hline $4 a$ & $32.14(52.07)$ & $>100$ & $>100$ & $>100$ & $>100$ & $>100$ & $>100$ & $>100$ & $86.77(92.17)$ \\
\hline $4 b$ & $65.59(48.62)$ & $>100$ & $>100$ & $>100$ & $>100$ & $>100$ & $>100$ & $>100$ & $94.86(92.17)$ \\
\hline $4 c$ & $35.12(>100)$ & $>100$ & $>100$ & $>100$ & $>100$ & $>100$ & $>100$ & $>100$ & $87.74(>100)$ \\
\hline $5 \mathbf{a}$ & $91.16(>100)$ & $66.96(>100)$ & $37.97(>100)$ & $12.94(52.62)$ & $39.85(>100)$ & $72.49(>100)$ & $34.54(>100)$ & $>100$ & $48.36(92.29)$ \\
\hline $5 b$ & $53.52(>100)$ & $68.48(>100)$ & $52.52(>100)$ & $21.66(41.14)$ & $>100$ & $56.83(>100)$ & $22.25(>100)$ & $>100$ & $51.91(89.49)$ \\
\hline $5 c$ & $58.52(>100)$ & $>100$ & $94.87(>100)$ & $19.62(>100)$ & $>100$ & $>100$ & $58.91(>100)$ & $>100$ & $70.94(>100)$ \\
\hline $6 a$ & $34.17(>100)$ & $>100$ & $51.40(>100)$ & $>100$ & $>100$ & $72.79(>100)$ & $27.24(57.73)$ & $75.33(>100)$ & $63.44(93.36)$ \\
\hline $6 \mathbf{b}$ & $21.31(47.18)$ & $>100$ & $46.48(>100)$ & $>100$ & $>100$ & $>100$ & $21.53(49.91)$ & $91.41(>100)$ & $61.13(83.18)$ \\
\hline $6 c$ & $25.34(52.70)$ & $>100$ & $91.37(>100)$ & $77.82(>100)$ & $71.19(>100)$ & $60.35(>100)$ & $24.81(>100)$ & $65.45(>100)$ & $57.86(93.33)$ \\
\hline $7 \mathbf{a}$ & $26.24(53.19)$ & $>100$ & $>100$ & $37.81(>100)$ & $>100$ & $63.76(>100)$ & $28.81(56.37)$ & $71.36(>100)$ & $58.11(86.02)$ \\
\hline $7 b$ & $>100$ & $>100$ & $>100$ & $>100$ & $46.84(>100)$ & $30.11(>100)$ & $44.97(69.85)$ & $57.36(>100)$ & $66.09(95.61)$ \\
\hline $7 c$ & $>100$ & $>100$ & $>100$ & $>100$ & $>100$ & $>100$ & $>100$ & $>100$ & $>100(>100)$ \\
\hline
\end{tabular}

aTI and $\mathrm{LC}_{50}$ mean-graph midpoint $(\mathrm{MG}-\mathrm{MID})=$ average sensitivity of all cell lines toward the test compounds.

improved antitumoral activity 4.5 timefold compared to those of the 1,3,4-oxadiazoles 1a and 1c. Also, concerning to the effect of the different alkylamino groups on activity we observed that the isopropylamino(methyl) and benzylamino(methyl) substituents were the best choice for an increase of activity.

\section{Antimicrobial activity}

Compounds 1a-c and their Mannich bases 2-7(a-c) were assayed against the bacteria Bacillus subtilis ATCC 2576, Escherichia coli ATCC 25922, Pseudomonas aeruginosa ATCC 13388, Sthaphylococus aureus ATCC 6538; and against the fungi Candida albicans ATCC 10231, Candida parapsilosis ATCC-22019 and Candida tropicalis ATCC28707.

The bioassays results for compounds 1a-c showed a potent antifungal activity for compound $\mathbf{1 b}$ against the Candida albicans fungi, with MIC value of $3.1 \mu \mathrm{g} \mathrm{mL}^{-1}$ This derivative and the compound 1a were weakly active towards the bacteria B. subtilis $\left(\mathrm{MIC}=250 \mu \mathrm{g} \mathrm{mL}^{-1}\right.$ ). For the Mannich bases 2-7(a-c) none of the derivatives presented activity at the maximum concentration tested ( $250 \mu \mathrm{g} \mathrm{mL}^{-1}$ ), which demonstrated that the introduction of alkylamino(methyl) groups at the oxadiazole ring of 1a-c did not contribute for antimicrobial activity.

In silico study

An in silico computational study of the synthesized 1-phenyl substituted-3-(3-alkylaminomethyl-2-thioxo-1,3,4oxadiazol-5-yl) $\beta$-carbolines 2-7(a-c) was performed by determination of Lipinski's parameters, topological polar surface area (TPSA) and percentage of absorption (\% ABS). Calculations were performed using "Molinspiration online property calculation toolkit" (http://www.molinspiration. com) ${ }^{20}$ and "OSIRIS property explorer" (www.organicchemistry.org/prog/peo). ${ }^{21}$ The percentage of absorption was estimated using equation: \% ABS $=109-0.345 \times$ TPSA, according to Zhao et al..$^{19}$ These data are shown in Table 3. 
Table 3. Lipinsk's parameters and \%ABS, TPSA, Log S for compounds 2-7 (a-c)

\begin{tabular}{|c|c|c|c|c|c|c|c|c|}
\hline \multirow[t]{2}{*}{ Comp } & \multirow[t]{2}{*}{$\% \mathrm{ABS}$} & \multirow{2}{*}{$\operatorname{TPSA}\left(\AA^{2}\right)$} & \multicolumn{5}{|c|}{ Lipinski's parameters } & \multirow[t]{2}{*}{$\log \mathrm{S}$} \\
\hline & & & nALH acceptors & nDLH donors & milogP & MW & $\mathrm{n}$ violations & \\
\hline $2 \mathbf{a}$ & 84.27 & 71.68 & 6 & 2 & 4.31 & 415.52 & 0 & -6.51 \\
\hline $2 \mathbf{b}$ & 68.46 & 117.50 & 9 & 2 & 4.27 & 460.52 & 0 & -6.97 \\
\hline $2 c$ & 83.15 & 74.91 & 7 & 2 & 4.42 & 458.59 & 0 & -6.55 \\
\hline $3 \mathbf{a}$ & 84.27 & 71.676 & 6 & 2 & 5.08 & 429.55 & 1 & -6.67 \\
\hline $3 \mathbf{b}$ & 68.46 & 117.5 & 9 & 2 & 5.04 & 474.55 & 1 & -7.13 \\
\hline $3 c$ & 83.15 & 74.914 & 7 & 2 & 5.18 & 472.62 & 1 & -6.71 \\
\hline $4 a$ & 84.27 & 71.68 & 6 & 2 & 5.55 & 455.59 & 1 & -7.41 \\
\hline $4 b$ & 68.46 & 117.50 & 9 & 2 & 5.50 & 500.58 & 2 & -6.38 \\
\hline $4 c$ & 83.15 & 74.91 & 7 & 2 & 5.65 & 498.66 & 1 & -7.44 \\
\hline $5 a$ & 84.27 & 71.68 & 6 & 2 & 5.04 & 463.57 & 1 & -7.16 \\
\hline $5 b$ & 68.46 & 117.50 & 9 & 2 & 5.00 & 508.56 & 1 & -6.38 \\
\hline $5 c$ & 83.15 & 74.91 & 7 & 2 & 5.14 & 506.64 & 2 & -7.19 \\
\hline $6 \mathbf{a}$ & 87.3 & 62.89 & 6 & 1 & 4.29 & 427.53 & 0 & -6.16 \\
\hline $6 b$ & 71.49 & 108.71 & 9 & 1 & 4.25 & 472.53 & 0 & -6.62 \\
\hline $6 c$ & 86.19 & 66.13 & 7 & 1 & 4.39 & 470.60 & 0 & -6.19 \\
\hline $7 \mathbf{a}$ & 84.12 & 72.12 & 7 & 1 & 3.73 & 443.53 & 0 & -5.54 \\
\hline $7 \mathbf{b}$ & 68.31 & 117.95 & 10 & 1 & 3.69 & 488.53 & 0 & -6.00 \\
\hline $7 c$ & 83.00 & 75.36 & 8 & 1 & 3.83 & 486.60 & 0 & -5.57 \\
\hline
\end{tabular}

In vivo absorption of the new synthesized derivatives was tentatively assessed by means of theoretical calculations following Lipinski's rule of five, which establishes that the absorption or permeation of an orally administered compound is more likely to be good if the drug satisfies the following criteria: $(i)$ hydrogen bond donors $\leq 5$ ( $\mathrm{OH}$ and $\mathrm{NH}$ groups); (ii) hydrogen bond acceptors $\leq 10$ ( $\mathrm{N}$ and $\mathrm{O}$ atoms); (iii) molecular weight $<500$; (iv) calculated $\log \mathrm{P}<5 .{ }^{18-20}$ Compounds violating more than one of these rules may present bioavailability problems.

Our results (Table 3) revealed that the Mannich base derivatives containing the isopropylamino(methyl) (2a-c), pyrrolidyl(methyl) (6a-c) and morpholyl(methyl) (7a-c) groups attached to the 1,3,4-oxadiazole ring presented lipophilicity minor than 5, with values between 3.69 and 4.41. On the other hand, the derivatives containing the butylamino(methyl) (3a-c), cyclohexylamino(methyl) (4a-c) and benzylamino(methyl) (5a-c) groups showed lipophilicity larger than 5.0, violating one of the Lipinski's rules.

Except for $\mathbf{4 b}, \mathbf{5 b}$ e $\mathbf{5} \mathbf{c}$ the molecular weight were minor than 500 (415.52 > MW < 498.65). All Mannich base derivatives have number of hydrogen bond acceptors $(n$ $\mathrm{ON}=6-10)$ and donors $(n-\mathrm{OHNH}=1-2)$ in agreement with Lipinski's rule. The calculated percent absorption (\% ABS) of all derivatives ranged between 68.31 and $87.30 \%$, indicating that these compounds have a good permeability in the cellular plasmatic membrane. In summary, in silico study pointed the Mannich bases synthesized in our work as potential candidates for new antitumor agents.

\section{Conclusions}

In conclusion, several 1 -substituted phenyl- $\beta$-carbolines containing a 3-alkylamino(methyl)-2-thioxo-1,3,4oxadiazolyl group were prepared and identified as novel antitumor agents. Compounds $\mathbf{2 a}, \mathbf{2 c}$ and $\mathbf{5} \mathbf{c}$ were the most active derivatives, exhibiting a broad spectrum antitumor activity at $\mathrm{GI}_{50}$ and TGI levels. Except for $\mathbf{3 b}, \mathbf{4 c}$ and $\mathbf{7 c}$, the Mannich base derivatives displayed higher anticancer activity than their corresponding precursors. For the most active Mannich bases 2a, 2c and $\mathbf{5 c}$, the introduction of an alkylamino(methyl) group at $N-3$ of the heterocyclic ring improved antitumor activity 4.5 timefold compared to those of the 1,3,4-oxadiazoles $\mathbf{1 a}$ and $\mathbf{1 c}$.

The antimicrobial bioassays results showed that, among all tested compounds, only compound $\mathbf{1 b}$ was active, displaying potent activity against the yeast $C$. albicans.

Finally, in silico study pointed the novel derivatives as potential candidates for new drugs. 


\section{Experimental}

General

${ }^{1} \mathrm{H}$ and ${ }^{13} \mathrm{C}$ spectra were recorded in a Varian spectrometer model Mercury plus BB at $300 \mathrm{MHz}$ and $75.5 \mathrm{MHz}$, respectively, with DMSO- $d_{6}$ as solvent and TMS as the internal standard. Mass spectra (MS) were recorded in a Thermoelectron Corporation Focus-DSQ II spectrometer. IR spectra were recorded on a BOMEM spectrometer model MB-100. For TLC, Merck precoated plates (silica gel 60 G254) were used. Silica gel 60 Merck (230-400 mesh) was used in column chromatography purification of some compounds. All reagents were purchased from commercial suppliers.

General procedure for preparation of 1-(substituted phenyl)3-[2-thioxo-3-alkylamino(methyl)-1,3,4-oxadiazol-5-yl] $\beta$-carbolines 2-7(a-c)

The 1-(substituted phenyl)-3-(2-thioxo-1,3,4-oxadiazol5-yl) $\beta$-carbolines 1a-c were prepared as previously reported. $^{14}$

To a solution of derivatives 1a-c $(0.5 \mathrm{mmol})$ in ethanol $(10 \mathrm{~mL})$ the primary or secondary amines $(0.5 \mathrm{mmol})$ were added, followed of the dropwise addition of formaldehyde $(37 \%, 3.0 \mathrm{mmol})$. The solution was stirred for $24 \mathrm{~h}$ at $60{ }^{\circ} \mathrm{C}$ and then, for $1 \mathrm{~h}$ at $0{ }^{\circ} \mathrm{C}$. The precipitated solid was filtered under vacuum suction and washed with cold ethanol. The characterization data of the Mannich bases obtained are given bellow.

1-Phenyl-3-[3-isopropylamino(methyl)-2-thioxo-1,3,4oxadiazol-5-yl] $\beta$-carboline (2a)

Yield: $62 \%$, mp 234.0-237.0 ${ }^{\circ} \mathrm{C}$. IR $(\mathrm{KBr}) \mathrm{v}_{\max } / \mathrm{cm}^{-1}$ : $3040(\mathrm{~N}-\mathrm{H}), 1614(\mathrm{C}=\mathrm{N}), 1360$ and $1239(\mathrm{C}=\mathrm{S}), 1565$, 1487 and $1464(\mathrm{C}=\mathrm{C})$. ${ }^{1} \mathrm{H}$ NMR $\left(300 \mathrm{MHz}, \mathrm{DMSO}-d_{6}\right)$ : $\delta 8.94$ (s, 1H, H-4), 8.47 (d, J 7.8 Hz, 1H, H-5), 7.35 (t, $J$ $7.8 \mathrm{~Hz}, 1 \mathrm{H}, \mathrm{H}-6)$, 7.57-7.73 (m, 5H, H-7, H-8, H-3', H-4' and $\mathrm{H}_{-} 5^{\prime}$ ), 12.00 (s, 1H, NH-9), 8.09 (d, J 6.9 Hz, 2H, H-2' and H-6'), 5.99 (brs, 2H, $\mathrm{CH}_{2}$-exocyclic), 11.43 (brs, 1H, NH-isopropyl), 4.60 (hep, J $6.9 \mathrm{~Hz}, 1 \mathrm{H}, \mathrm{CH}$-isopropyl), 1.22 (d, J 6.9 Hz, 6H, CH -isopropyl). ${ }^{13} \mathrm{C} \mathrm{NMR}(75.5 \mathrm{MHz}$, DMSO- $\left.d_{6}\right): \delta 141.06(\mathrm{C}-1), 138.9(\mathrm{C}-3), 115.9(\mathrm{C}-4), 128.8$ (C-4a), 121.1 (C-4b), 122.2 (C-5), 120.5 (C-6), 129.2 (C7), 112.8 (C-8), 140.4 (C-8a), 133.9 (C-9a), 129.9 (C-1'), 128.5 (C-2'/6'), 129.0 (C-3'/5'), 128.9 (C-4'), 174.3 (C-2’'), 159.7 (C-5”), 64.6 ( $\mathrm{CH}_{2}$-exocyclic), 46.2 (CH-isopropyl), $18.9\left(\mathrm{CH}_{3}\right.$-isopropyl). EIMS, $70 \mathrm{eV}, \mathrm{m} / \mathrm{z}$ (rel. int., \%): 415 (10, M+), 344 (10), 271 (20), 243 (100), 121 (5), $100(5)$.
1-(3-Nitrophenyl)-3-[3-isopropylamino(methyl)-2-thioxo1,3,4-oxadiazol-5-yl] $\beta$-carboline (2b)

Yield: $89 \%$, mp 236.0- $237.0{ }^{\circ} \mathrm{C}$. IR (KBr) $v_{\max } / \mathrm{cm}^{-1}$ : $3054(\mathrm{~N}-\mathrm{H}), 1611(\mathrm{C}=\mathrm{N}), 1350$ and $1239(\mathrm{C}=\mathrm{S}), 1566$, 1485 and $1465(\mathrm{C}=\mathrm{C}) .{ }^{1} \mathrm{H}$ NMR $\left(300 \mathrm{MHz}, \mathrm{DMSO}-d_{6}\right): \delta$ 9.01 (s, 1H, H-4), 8.55 (d, J 7.8 Hz, 1H, H-5), 7.37 (t, J 7.8 Hz, 1H, H-6), 7.63-7.73 (m, 2H, H-7, H-8), 12.17 (s, 1H, 9-NH), 8.83 (s, 1H, H-2'), 8.51 (d, J 7.8 Hz, H-4'), 7.97 (t, J 7.8 Hz, H-5'), 8.44 (dd, J 7.8 Hz, 1.8 Hz, H-6'), 6.00 (brs, 2H, $\mathrm{CH}_{2}$-exocyclic), 11.46 (brs, 1H, NH-isopropyl), 4.63 (hep, J 6.9 Hz, 1H, CH-isopropyl), 1.23 (d, J 6.9 Hz, $6 \mathrm{H}, \mathrm{CH}_{3}$-isopropyl). ${ }^{13} \mathrm{C} \mathrm{NMR}\left(75.5 \mathrm{MHz}, \mathrm{DMSO}-d_{6}\right): \delta$ 141.7 (C-1), 137.9 (C-3), 116.7 (C-4), 130.5 (C-4a), 121.0 (C-4b), 122.4 (C-5), 120.7 (C-6), 129.2 (C-7), 112.7 (C-8), 139.09 (C-8a), 139.0 (C-9a), 134.1 (C-1'), 123.3 (C-2'), 148.3 (C-3'), 123.6 (C-4'), 130.5 (C-5'), 134.9 (C-6’), 174.3 (C-2”), 159.9 (C-5”), 64.6 ( $\mathrm{CH}_{2}$-exocyclic), 46.2 (CH-isopropyl), 18.9 ( $\mathrm{CH}_{3}$-isopropyl). EIMS, $70 \mathrm{eV}, \mathrm{m} / \mathrm{z}$ (rel. int., \%): 460 (5, M+), 344 (15), 289 (95), 242 (100), 121(30), 100 (75).

1-(4-N,N-Dimethylaminophenyl)-3-[3-isopropylamino (methyl)-2-thioxo-1,3,4-oxadiazol-5-yl] $\beta$-carboline (2c)

Yield: $90 \%, \mathrm{mp} 160.0-161.0{ }^{\circ} \mathrm{C}$. IR $(\mathrm{KBr}) \mathrm{v}_{\max } / \mathrm{cm}^{-1}$ : $3085(\mathrm{~N}-\mathrm{H}), 1609(\mathrm{C}=\mathrm{N}), 1365$ and $1240(\mathrm{C}=\mathrm{S}), 1557$, 1488 and $1466(\mathrm{C}=\mathrm{C}) .{ }^{1} \mathrm{H}$ NMR $\left(300 \mathrm{MHz}, \mathrm{DMSO}-d_{6}\right)$ : $\delta 8.82(\mathrm{~s}, 1 \mathrm{H}, \mathrm{H}-4), 8.42(\mathrm{~d}, J 7.8 \mathrm{~Hz}, 1 \mathrm{H}, \mathrm{H}-5), 7.32$ (t, $J 7.8 \mathrm{~Hz}, \mathrm{H}-6), 7.60$ (t, J 7.8 Hz, H-7), 7.72 (d, J $7.8 \mathrm{~Hz}$, H-8), 11.87 (s, 1H, 9-NH), 7.95 (d, J 8.7 Hz, 2H, H-2' and H-6'), 6.96 (d, J 8.7 Hz, 2H, H-3' and H-5'), 3.05 (s, 6H, $\left.\mathrm{N}\left(\mathrm{CH}_{3}\right)_{2}\right), 5.98$ (brs, 2H, $\mathrm{CH}_{2}$ - exocyclic), 11.40 (brs, $1 \mathrm{H}$, NH-isopropyl), 4.61 (hep, J $6.6 \mathrm{~Hz}, 1 \mathrm{H}, \mathrm{CH}$-isopropyl), 1.24 (d, J $6.6 \mathrm{~Hz}, 6 \mathrm{H}, \mathrm{CH}_{3}$-isopropyl). ${ }^{13} \mathrm{C}$ NMR $(75.5$ MHz, DMSO- $\left.d_{6}\right): \delta 141.5(\mathrm{C}-1), 138.7$ (C-3), 114.7 (C4), 124.9 (C-4a), 121.2 (C-4b), 122.0 (C-5), 120.3 (C-6), 128.6 (C-7), 112.8 (C-8), 141.3 (C-8a), 133.5 (C-9a), 129.4 (C-1'), 129.3 (C-2'), 112.2 (C-3'), 150.8 (C-4'), 112.2 (C-5'), 129.3 (C-6'), 40.0 - N( $\left.\mathrm{CH}_{3}\right)_{2}, 174.3$ (C-2”), 166.7 (C-5"), 64.6 ( $\mathrm{CH}_{2}$-exocyclic), 46.2 (CH-isopropyl), 19.0 ( $\mathrm{CH}_{3}$-isopropyl). EIMS, $70 \mathrm{eV}, \mathrm{m} / \mathrm{z}$ (rel. int., \%): 458 (5, $\mathrm{M}^{+\cdot)}, 344$ (10), 287 (100), 242 (45), 121 (25), 100 (45).

1-Phenyl-3-[3-butylamino(methyl)-2-thioxo-1,3,4oxadiazol-5-yl] $\beta$-carboline (3a)

Yield: $83 \%$, mp 212.0-215.0 ${ }^{\circ} \mathrm{C}$. IR (KBr) $v_{\max } / \mathrm{cm}^{-1}$ : $3057(\mathrm{~N}-\mathrm{H}), 1618(\mathrm{C}=\mathrm{N}), 1394$ and $1238(\mathrm{C}=\mathrm{S}), 1566$, 1492 and $1456(\mathrm{C}=\mathrm{C}) .{ }^{1} \mathrm{H}$ NMR $\left(300 \mathrm{MHz}, \mathrm{DMSO}-d_{6}\right)$ : $\delta 8.93$ (s, 1H, H-4), 8.46 (d, J $7.8 \mathrm{~Hz}, 1 \mathrm{H}, \mathrm{H}-5), 7.35$ (t, J 7.8 Hz, 1H, H-6), 7.57-7.73 (m, 5H, H-7, H-8, H-3', H-4' and $\mathrm{H}-5$ '), 11.99 (s, 1H, 9-NH), 8.08 (d, J 6.9 Hz, 2H, H-2' and H-6'), 5.92 (brs, 2H, $\mathrm{CH}_{2}$-exocyclic), 11.44 (brs, $1 \mathrm{H}$, 
NH-butyl), 3.58 (t, J 7.2 Hz, 2H, $\mathrm{CH}_{2}$-butyl), 1.56 (quint., $J 7.2 \mathrm{~Hz}, 2 \mathrm{H}, \mathrm{CH}_{2}$-butyl), 1.31 (sext., J $7.2 \mathrm{~Hz}, 2 \mathrm{H}, \mathrm{CH}_{2}$ butyl), 0.90 (t, $J 7.2 \mathrm{~Hz}, 3 \mathrm{H}, \mathrm{CH}_{3}$-butyl). ${ }^{13} \mathrm{C}$ NMR $(75.5$ MHz, DMSO- $\left.d_{6}\right): \delta 141.6(\mathrm{C}-1), 138.9(\mathrm{C}-3), 115.9(\mathrm{C}-$ 4), 129.9 (C-4a), 121.1 (C-4b), 122.2 (C-5), 120.4 (C-6), 129.2 (C-7), 112.8 (C-8), 140.5 (C-8a), 137.5 (C-9a), 133.9 (C-1'), 128.5 (C-2'/6'), 129.0 (C-3'/5'), 128.9 (C-4'), 175.5 (C-2”), 160.5 (C-5”), 68.0 ( $\mathrm{CH}_{2}$-exocyclic), 43.9 $\left(\mathrm{CH}_{2}\right.$-butyl), $28.4\left(\mathrm{CH}_{2}\right.$ - butyl $), 19.4\left(\mathrm{CH}_{2}-\right.$ butyl $), 13.6$ $\left(\mathrm{CH}_{3}\right.$-butyl). EIMS, $70 \mathrm{eV}, \mathrm{m} / z$ (rel. int., \%): $429\left(5, \mathrm{M}^{+}\right)$, 344 (25), 243 (100), 157 (15), 102 (15).

1-(3-Nitrophenyl)-3-[3-butylamino(methyl)-2-thioxo-1,3,4oxadiazol-5-yl] $\beta$-carboline (3b)

Yield: $65 \%$, mp 230.0-235.0 ${ }^{\circ} \mathrm{C}$. IR (KBr) $v_{\max } / \mathrm{cm}^{-1}$ : $3053(\mathrm{~N}-\mathrm{H}), 1612(\mathrm{C}=\mathrm{N}), 1345$ and $1238(\mathrm{C}=\mathrm{S}), 1565$ and $1454(\mathrm{C}=\mathrm{C}) .{ }^{1} \mathrm{H}$ NMR (300 MHz, DMSO- $\left.d_{6}\right): \delta 9.00$ (s, $1 \mathrm{H}, \mathrm{H}-4) ; 8.55$ (d, J 7.8 Hz, 1H, H-5), 7.37 (t, J 7.8 Hz, 1H, H-6), 7.63 - 7.73 (m, 2H, H-7, H-8), 12.17 (s, 1H, 9-NH), 8.83 (s, $\left.1 \mathrm{H}, \mathrm{H}-2^{\prime}\right), 8.50$ (d, $\left.J 8.0 \mathrm{~Hz}, 1 \mathrm{H}, \mathrm{H}-4^{\prime}\right), 7.96$ (t, $J$ $8.0 \mathrm{~Hz}, 1 \mathrm{H}, \mathrm{H}-5^{\prime}$ ), 8.43 (dd, $J 8.0$ and $2.1 \mathrm{~Hz}, 1 \mathrm{H}, \mathrm{H}-6^{\prime}$ ), 5.98 (brs, $2 \mathrm{H}, \mathrm{CH}_{2}$-exocyclic), 11.47 (brs, $1 \mathrm{H}, \mathrm{NH}-$ butyl), 3.59 (t, J 7.2 Hz, 2H, CH $\mathrm{CH}_{2}$-butyl), 1.59 (quint., J 7.2 Hz, $2 \mathrm{H}$, $\mathrm{CH}_{2}$-butyl), 1.31 (sext., J 7.2 Hz, 2H, $\mathrm{CH}_{2}$-butyl), 0.90 (t, $J$ $7.2 \mathrm{~Hz}, 3 \mathrm{H}, \mathrm{CH}_{3}$-butyl). ${ }^{13} \mathrm{C}$ NMR $\left(75.5 \mathrm{MHz}\right.$, DMSO- $\left.d_{6}\right)$ : $\delta 141.7$ (C-1), 138.9 (C-3), 116.7 (C-4), $121.0\left(\mathrm{C}_{0}-4 \mathrm{~b}\right)$, 123.6 (C-5), 120.7 (C-6), 129.2 (C-7), 112.7 (C-8), 139.1 (C-8a), 137.8 (C-9a), 134.1 (C-1'), 123.4 (C-2'), 148.3 (C3'), 122.4 (C-4'), 130.5 (C-5'), 134.8 (C-6'), 175.4 (C-2”), 158.5 (C-5"), $68.5\left(\mathrm{CH}_{2}\right.$-exocyclic), 44.1 ( $\mathrm{CH}_{2}$-butyl), 28.3 $\left(\mathrm{CH}_{2}\right.$-butyl $), 19.5\left(\mathrm{CH}_{2}\right.$-butyl $), 13.6\left(\mathrm{CH}_{3}\right.$-butyl $)$. EIMS, $70 \mathrm{eV}, \mathrm{m} / \mathrm{z}$ (rel. int., \%): $474\left(5, \mathrm{M}^{+}\right), 242$ (15), 157 (95), 102 (75), 57 (100).

1-(4-N,N-Dimethylaminophenyl)-3-[3-butylamino(methyl)2-thioxo-1,3,4-oxadiazol-5-yl] $\beta$-carboline (3c)

Yield: $92 \%$, mp $214.0-216^{\circ} \mathrm{C}$. IR (KBr) $v_{\max } / \mathrm{cm}^{-1}: 3262$ $(\mathrm{N}-\mathrm{H}) ; 1608(\mathrm{C}=\mathrm{N}) ; 1348$ and $1229(\mathrm{C}=\mathrm{S}), 1558,1493$ and $1452(\mathrm{C}=\mathrm{C}) .{ }^{1} \mathrm{H}$ NMR $\left(300 \mathrm{MHz}, \mathrm{DMSO}-d_{6}\right): \delta 8.80(\mathrm{~s}$, $1 \mathrm{H}, \mathrm{H}-4), 8.42$ (d, J 7.8 Hz, 1H, H-5), 7.32 (t, J $7.8 \mathrm{~Hz}, 1 \mathrm{H}$, H-6), 7.60 (t, J 7.8 Hz, 1H, H-7), 7.71 (d, J 7.8 Hz, 1H, H-8), $11.86(\mathrm{~s}, 1 \mathrm{H}, 9-\mathrm{NH}), 7.95(\mathrm{~d}, J 8.7 \mathrm{~Hz}, 1 \mathrm{H}, \mathrm{H}-2$ ') 6.96 (d, $J 8.7 \mathrm{~Hz}, 1 \mathrm{H}, \mathrm{H}-3$ '), 6.96 (d, J 8.7 Hz, 1H, H-5'), 7.95 (d, $J 8.7 \mathrm{~Hz}, 1 \mathrm{H}, \mathrm{H}-6$ '), 3.05 (s, 6H, N( $\left.\left.\mathrm{CH}_{3}\right)_{2}\right), 5.92$ (brs, $2 \mathrm{H}$, $\mathrm{CH}_{2}$-exocyclic), 11.41 (brs, 1H, NH-butyl), 3.59 (t, $J 7.3$ $\mathrm{Hz}, 2 \mathrm{H}, \mathrm{CH}_{2}$-butyl), 1.57 (quint., J 7.3 Hz, $2 \mathrm{H}, \mathrm{CH}_{2}$-butyl), 1.32 (sext., J $7.3 \mathrm{~Hz}, 2 \mathrm{H}, \mathrm{CH}_{2}$-butyl), 0.92 (t, $J 7.3 \mathrm{~Hz}, 3 \mathrm{H}$, $\mathrm{CH}_{3}$-butyl). ${ }^{13} \mathrm{C}$ NMR $\left(75.5 \mathrm{MHz}, \mathrm{DMSO}-d_{6}\right): \delta 141.4(\mathrm{C}-$ 1), 138.7 (C-3), 114.7 (C-4), 124.9 (C-4a), 121.2 (C-4b), 122.0 (C-5), 120.3 (C-6), 128.6 (C-7), 112.8 (C-8), 141.3 (C-8a), 129.3 (C-9a), 133.5 (C-1'), 129.3 (C-2'), 112.2
(C-3'), 150.8 (C-4'), 112.2 -(C-5'), 129.3 (C-6'), 175.5 (C-2"), 164.6 (C-5"), $40.0-\left(\mathrm{CH}_{3}\right)_{2}, 68.2\left(\mathrm{CH}_{2}\right.$-exocyclic), $44.0\left(\mathrm{CH}_{2}\right.$-butyl), $28.5\left(\mathrm{CH}_{2}\right.$-butyl), $19.5\left(\mathrm{CH}_{2}\right.$-butyl $), 13.7$ $\left(\mathrm{CH}_{3}\right.$-butyl). EIMS, $70 \mathrm{eV}, \mathrm{m} / \mathrm{z}$ (rel. int., \%): $472\left(5, \mathrm{M}^{+}\right)$, 242 (25), 157 (70), 84 (25), 76 (100).

1-Phenyl-3-[3-cyclohexylamino(methyl)-2-thioxo-1,3,4oxadiazol-5-yl] $\beta$-carboline (4a)

Yield: 74\%, mp 222.0-224.0 ${ }^{\circ} \mathrm{C}$. IR (KBr) $v_{\max } / \mathrm{cm}^{-1}$ : $3042(\mathrm{~N}-\mathrm{H}), 1613(\mathrm{C}=\mathrm{N}), 1372$ and $1237(\mathrm{C}=\mathrm{S}), 1564$, 1484 and $1453(\mathrm{C}=\mathrm{C}) .{ }^{1} \mathrm{H}$ NMR $\left(300 \mathrm{MHz}, \mathrm{DMSO}-d_{6}\right): \delta$ 8.94 (s, 1H, H-4), 8.45 (d, J 7.8 Hz, 1H, H-5), 7.35 (t, J 7.8 $\mathrm{Hz}, 1 \mathrm{H}, \mathrm{H}-6), 7.58$ - 7.73 (m, 5H, H-7, H-8, H-3', H-4' and H-5'), 12.00 (s, 1H, 9-NH), 8.10 (d, J 6.9 Hz, 2H, H-2' and H-6'), 6.00 (brs, 2H, $\mathrm{CH}_{2}$-exocyclic), 11.40 (brs, $1 \mathrm{H}, \mathrm{NH}-$ cyclohexyl), 4.21 (m, 1H, CH-cyclohexyl), 1.03-1.84 (m, $10 \mathrm{H}, \mathrm{CH}_{2}$-cyclohexyl). ${ }^{13} \mathrm{C}$ NMR $\left(75.5 \mathrm{MHz}\right.$, DMSO- $\left.d_{6}\right): \delta$ 141.6 (C-1), 138.9 (C-3), 115.8 (C-4), 128.7 (C-4a), 121.1 (C-4b), 122.2 (C-5), 120.4 (C-6), 129.2 (C-7), 112.8 (C-8), 140.3 (C-8a), 133.8 (C-9a), 130.0 (C-1'), 128.5 (C-2'/6'), 128.9 (C-3'/5'), 128.8 (C-4'), 177.6 (C-2”), 166.8 (C-5”), $65.3\left(\mathrm{CH}_{2}\right.$-exocyclic), 53.7 (CH-cyclohexyl), $29.1\left(\mathrm{CH}_{2}-\right.$ cyclohexyl), $25.0\left(\mathrm{CH}_{2}\right.$-cyclohexyl). EIMS, $70 \mathrm{eV}, \mathrm{m} / z$ (rel. int., \%): $455\left(5, \mathrm{M}^{+}\right), 243$ (100), 183 (10), 102 (25), 55 (30).

1-(3-Nitrophenyl)-3-[3-cyclohexylamino(methyl)-2-thioxo1,3,4-oxadiazol-5-yl] $\beta$-carboline (4b)

Yield: 83\%, mp 222.0-223.0 ${ }^{\circ} \mathrm{C}$. IR (KBr) $v_{\max } / \mathrm{cm}^{-1}$ : $3064(\mathrm{~N}-\mathrm{H}), 1613(\mathrm{C}=\mathrm{N}), 1346$ and $1239(\mathrm{C}=\mathrm{S}), 1559$, 1485 and $1459(\mathrm{C}=\mathrm{C}) .{ }^{1} \mathrm{H}$ NMR (300 MHz, DMSO- $\left.d_{6}\right)$ : $\delta 9.00$ (s, 1H, H-4), 8.55 (d, J 7.8 Hz, 1H, H-5), 7.37 (t, $J$ $7.8 \mathrm{~Hz}, 1 \mathrm{H}, \mathrm{H}-6), 7.63-7.73$ (m, 2H, H-7 and H-8), 12.17 (s, 1H, 9-NH), 8.82 (brs, 1H, H-2'), 8.50 (d, J $8.1 \mathrm{~Hz}, 1 \mathrm{H}$, H-4') 7.97 (t, J $8.1 \mathrm{~Hz}, 1 \mathrm{H}, \mathrm{H}-5$ '), 8.44 (d, J $8.1 \mathrm{~Hz}, 1 \mathrm{H}$, H-6'), 6.02 (brs, 2H, $\mathrm{CH}_{2}$-exocyclic), 11.46 (brs, $1 \mathrm{H}, \mathrm{NH}-$ cyclohexyl-), 4.22 (m, 1H, CH-cyclohexyl), 1.03-1.85 (m, $10 \mathrm{H}, \mathrm{CH}_{2}$-cyclohexyl). ${ }^{13} \mathrm{C} \mathrm{NMR}\left(75.5 \mathrm{MHz}, \mathrm{DMSO}-d_{6}\right): \delta$ 141.7 (C-1), 139.1 (C-3), 116.7 (C-4), 121.0 (C-4b), 122.4 (C-5), 120.6 (C-6), 129.2 (C-7), 112.7 (C-8), 139.7 (C-8a), 137.9 (C-9a), 134.1 (C-1'), 123.4 (C-2'), 148.4 (C-3'), 123.6 (C-4'), 130.5 (C-5'), 134.9 (C-6'), 174.2 (C-2”), 164.9 (C-5"), 65.4 ( $\mathrm{CH}_{2}$-exocyclic), 53.8 (CH-cyclohexyl), $28.9\left(\mathrm{CH}_{2}\right.$-cyclohexyl), $24.9\left(\mathrm{CH}_{2}\right.$-cyclohexyl), $25.1\left(\mathrm{CH}_{2}-\right.$ cyclohexyl), $24.6\left(\mathrm{CH}_{2}\right.$-cyclohexyl). EIMS, $70 \mathrm{eV}, \mathrm{m} / z$ (rel. int., \%): 500 (5, $\left.\mathrm{M}^{+}\right), 242$ (60), 183 (30), 102 (100), 55 (90).

1-(4-N,N-Dimethylaminophenyl-3-[3-cyclohexylamino (methyl)-2-thioxo-1,3,4-oxadiazol-5-yl] $\beta$-carboline (4c)

Yield: $70 \%$, mp 173.0-175.0 ${ }^{\circ} \mathrm{C}$. IR (KBr) $v_{\max } / \mathrm{cm}^{-1}$ : $3083(\mathrm{~N}-\mathrm{H}), 1609(\mathrm{C}=\mathrm{N}), 1361$ and $1240(\mathrm{C}=\mathrm{S}), 1555$, 1489 and $1450(\mathrm{C}=\mathrm{C}) .{ }^{1} \mathrm{H}$ NMR (300 MHz, DMSO- $d_{6}$ ): 
$\delta 8.81$ (s, 1H, H-4), 8.42 (d, J $7.8 \mathrm{~Hz}, 1 \mathrm{H}, \mathrm{H}-5), 7.32$ (t, $J 7.8 \mathrm{~Hz}, 1 \mathrm{H}, \mathrm{H}-6), 7.60$ (t, J $7.8 \mathrm{~Hz}, 1 \mathrm{H}, \mathrm{H}-7$ ), 7.71 (d, $J 9.0 \mathrm{~Hz}, 1 \mathrm{H}, \mathrm{H}-8), 11.88$ (s, 1H, 9 -NH), 7.97 (d, J 9.0 Hz, 1H, H-2'), 6.96 (d, J 9.0 Hz, 1H, H-3'), 6.96 (d, $J$ $9.0 \mathrm{~Hz}, 1 \mathrm{H}, \mathrm{H}-5$ '), 7.97 (d, J 9.0 Hz, 1H, H-6'), 3.05 (s, $\left.6 \mathrm{H}, \mathrm{N}\left(\mathrm{CH}_{3}\right)_{2}\right), 5.99$ (brs, $2 \mathrm{H}, \mathrm{CH}_{2}$-exocyclic), 11.41 (brs, 1H, NH-cyclohexyl), 4.21 (m, 1H, CH-cyclohexyl), 1.03$1.84\left(\mathrm{~m}, 10 \mathrm{H}, \mathrm{CH}_{2}\right.$-cyclohexyl). ${ }^{13} \mathrm{C}$ NMR $(75.5 \mathrm{MHz}$, DMSO- $\left.d_{6}\right): \delta 141.4$ (C-1), 138.7 (C-3), 114.6 (C-4), 124.9 (C-4a), 121.2 (C-4b), 122.0 (C-5), 120.3 (C-6), 128.5 (C7), 112.7 (C-8), 141.2 (C-8a), 133.4 (C-9a), 129.4 (C-1'), 129.3 (C-2'), 112.1 (C-3'), 150.8 (C-4'), 112.1 (C-5'), 129.3 (C-6'), 174.2 (C-2"), $39.9-\mathrm{N}\left(\mathrm{CH}_{3}\right)_{2}, 65.4\left(\mathrm{CH}_{2}-\right.$ exocyclic), 53.7 (CH-cyclohexyl), $29.1\left(\mathrm{CH}_{2}\right.$-cyclohexyl), $25.0\left(\mathrm{CH}_{2}\right.$-cyclohexyl). EIMS, $70 \mathrm{eV}, \mathrm{m} / z$ (rel. int., \%): 498 $\left(5, \mathrm{M}^{+}\right), 183$ (5), 83 (55), 57 (100).

1-Phenyl-3-[3-benzylamino(methyl)-2-thioxo-1,3,4oxadiazol-5-yl] $\beta$-carboline (5a)

Yield: $79 \%$, mp 225.0-228.0 ${ }^{\circ} \mathrm{C}$. IR $(\mathrm{KBr}) \mathrm{v}_{\max } / \mathrm{cm}^{-1}$ : $3062(\mathrm{~N}-\mathrm{H}), 1618(\mathrm{C}=\mathrm{N}), 1396$ and $1237(\mathrm{C}=\mathrm{S}), 1565$, 1493, and $1454(\mathrm{C}=\mathrm{C}) .{ }^{1} \mathrm{H}$ NMR (300 MHz, DMSO- $d_{6}$ ): $\delta 8.91$ (s, 1H, H-4), 8.45 (d, J 7.8 Hz, 1H, H-5), 7.31-7.38 (m, 1H, H-6), 7.60-7.64 (m, 1H, H-7), 7.70 (d, J 7.8 Hz, 1H, H-8), 11.96 (brs, 1H, 9-NH), 7.89 (d, J $6.9 \mathrm{~Hz}, 2 \mathrm{H}$, H-2' and H-6'), 7.60 - 7.64 (m, 3H, H-3', H-4' and H-5'), 5.78 (brs, $2 \mathrm{H}, \mathrm{CH}_{2}$-exocyclic), 11.74 (brs, $1 \mathrm{H}, \mathrm{NH}-$ benzyl), 4.85 (s, 2H, $\mathrm{CH}_{2}$-benzyl), 7.31-7.38 (m, 3H, CH-benzyl), 7.42 (d, J 3.6 Hz, 2H, CH-benzyl). ${ }^{13} \mathrm{C}$ NMR $(75.5 \mathrm{MHz}$, DMSO- $\left.d_{6}\right): \delta 141.6$ (C-1), 138.6 (C-3), 115.9 (C-4), 129.9 (C-4a), 121.0 (C-4b), 122.2 (C-5), 120.4 (C-6), 129.1 (C7), 112.7 (C-8), 140.4 (C-8a), 137.4 (C-9a), 133.9 (C-1'), 128.4 (C-2'/6'), 129.0 (C-3'/5'), 128.9 (C-4'), 175.1 (C-2”), 160.1 (C-5"), 67.7 ( $\mathrm{CH}_{2}$-exocyclic), $47.7\left(\mathrm{CH}_{2}\right.$-benzyl), 135.4 (C-benzyl), 128.2 (CH-benzyl), 128.8 (CH-benzyl), 128.0 (CH-benzyl). EIMS, $70 \mathrm{eV}, \mathrm{m} / \mathrm{z}$ (rel. int., \%): 463 $\left(10, \mathrm{M}^{+}\right), 344$ (95), 242 (45), 191 (35), 91 (100).

1-(3-Nitrophenyl)-3-[3-benzylamino(methyl)-2-thioxo1,3,4-oxadiazol-5-yl] $\beta$-carboline (5b)

Yield: $77 \%$, mp 216.0-220.0 ${ }^{\circ} \mathrm{C}$. IR (KBr) $v_{\max } / \mathrm{cm}^{-1}$ : $3063(\mathrm{~N}-\mathrm{H}), 1621(\mathrm{C}=\mathrm{N}), 1348$ and $1240(\mathrm{C}=\mathrm{S}), 1566$, 1496 and $1454(\mathrm{C}=\mathrm{C}) .{ }^{1} \mathrm{H}$ NMR (300 MHz, DMSO- $\left.d_{6}\right)$ : $\delta$ 8.98 (s, 1H, H-4), 8.48 (d, J 7.5 Hz, 1H, H-5), 7.30-7.40 (m, 1H, H-6), $7.62-7.71$ (m, 2H, H-7 and H-8), 12.14 (s, $1 \mathrm{H}, 9-\mathrm{NH}), 8.71$ (brs, $1 \mathrm{H}, \mathrm{H}-2$ '), 8.43 (dd, J 7.2 and $1.8 \mathrm{~Hz}$, 1H, H-4'), 7.91 (t, J 7.2 Hz, 1H, H-5'), 8.32 (d, J 7.2 Hz, 1H, H-6'), 5.79 (brs, 2H, $\mathrm{CH}_{2}$-exocyclic), 11.78 (brs, $1 \mathrm{H}$, NH-benzyl), 4.82 (s, 2H, $\mathrm{CH}_{2}$-benzyl), 7.29-7.40 (m, 5H, CH-benzyl). ${ }^{13} \mathrm{C}$ NMR $\left(75.5 \mathrm{MHz}\right.$, DMSO- $\left.d_{6}\right): \delta 142.1$ (C1), 138.8 (C-3), 116.7 (C- 4), 127.8 (C-4a), 121.0 (C-4b),
122.4 (C-5), 120.7 (C-6), 129.2 (C-7), 112.6 (C-8), 141.6 (C-8a), 134.8 (C-9a), 134.1 (C-1'), 123.2 (C-2'), 148.2 (C3'), 123.6 (C-4'), 130.4 (C-5'), 135.4 (C-6'), 176.4 (C-2”), 160.2 (C-5”), $67.8\left(\mathrm{CH}_{2}\right.$-exocyclic), $47.7\left(\mathrm{CH}_{2}\right.$-benzyl), 137.9 (C-benzyl), 128.0 (CH-benzyl), 128.6 (CH-benzyl), 127.8 (CH-benzyl). EIMS, $70 \mathrm{eV}, \mathrm{m} / z$ (rel. int., \%): 509 (5, $\left.\mathrm{M}^{+}\right), 191$ (10), 91 (100), 65 (15).

1-(4-N,N-Dimethylaminophenyl)-3-[3-benzylamino (methyl)-2-thioxo-1,3,4-oxadiazol-5-yl] $\beta$-carboline (5c)

Yield: $86 \%$, mp 200.0-205.0 ${ }^{\circ} \mathrm{C}$. IR ( $\left.\mathrm{KBr}\right) v_{\max } / \mathrm{cm}^{-1}$ : $3086(\mathrm{~N}-\mathrm{H}), 1607(\mathrm{C}=\mathrm{N}), 1363$ and $1241(\mathrm{C}=\mathrm{S}), 1558$, 1495 and $1443(\mathrm{C}=\mathrm{C}) .{ }^{1} \mathrm{H}$ NMR (300 MHz, DMSO- $d_{6}$ ): $\delta 8.79$ (s, 1H, H-4), 8.40 (d, J 7.8 Hz, 1H, H-5), 7.28-7.39 (m, 1H, H-6), 7.59 (t, J 7.8 Hz, 1H, H-7), 7.68-7.76 (m, 3H, H-8, H-2' and H-6'), 11.84 (s, 1H, 9-NH), 6.86 (d, J 8.4 Hz, $2 \mathrm{H}, \mathrm{H}-3$ ' and $\mathrm{H}-5$ '), 3.06 (s, 6H, N($\left.\left(\mathrm{CH}_{3}\right)_{2}\right), 5.80$ (brs, $2 \mathrm{H}$, $\mathrm{CH}_{2}$-exocyclic), 4.86 (s, 2H, $\mathrm{CH}_{2}$-benzyl), 7.43 (d, J $4.5 \mathrm{~Hz}$, CH-benzyl), 7.28-7.39 (m, 3H, CH-benzyl). ${ }^{13} \mathrm{C} \mathrm{NMR}$ (75.5 MHz, DMSO- $\left.d_{6}\right)$ : $\delta 141.4$ (C-1), 135.4 (C-3), 114.7 (C-4), 124.8 (C-4a), 121.2 (C-4b), 122.0 (C-5), 120.3 (C-6), 128.5 (C-7), 112.8 (C-8), 141.1 (C-8a), 133.4 (C-9a), 129.3 (C-1'), 129.1 (C-2'/6'), 112.1 (C-3'/5'), 150.7 (C-4'), 175.1 (C-2'), 160.1 (C-5"), $40.0-\mathrm{N}\left(\mathrm{CH}_{3}\right)_{2}, 67.8\left(\mathrm{CH}_{2}\right.$-exocyclic $), 47.8$ ( $\mathrm{CH}_{2}$-benzyl), 138.4 (C-benzyl), 128.8 (CH-benzyl), 128.2 (CH-benzyl), 127.9 (CH-benzyl). EIMS, $70 \mathrm{eV}, \mathrm{m} / \mathrm{z}$ (rel. int., \%): $506\left(5, \mathrm{M}^{+}\right), 242$ (45), 191 (35), 91 (100).

1-Phenyl-3-[3-pyrrolidylamino(methyl)-2-thioxo-1,3,4oxadiazol-5-yl] $\beta$-carboline (6a)

Yield: 55\%, mp 207.0-209.0 ${ }^{\circ} \mathrm{C}$. IR (KBr) $v_{\max } / \mathrm{cm}^{-1}$ : $3280(\mathrm{~N}-\mathrm{H}), 1622(\mathrm{C}=\mathrm{N}), 1374$ and $1243(\mathrm{C}=\mathrm{S}), 1567$, 1496, 1445 and $1415(\mathrm{C}=\mathrm{C}) .{ }^{1} \mathrm{H}$ NMR $(300 \mathrm{MHz}$, DMSO- $\left.d_{6}\right): \delta 8.87(\mathrm{~s}, 1 \mathrm{H}, \mathrm{H}-4), 8.50(\mathrm{~d}, J 7.8 \mathrm{~Hz}, 1 \mathrm{H}$, H-5), 7.35 (t, J 7.8 Hz, 1H, H-6), 7.57 - 7.72 (m, H-7, H-8, H-3', H- 4' and H-5'), 11.99 (brs, 1H, 9-NH), 8.05 (d, J $6.9 \mathrm{~Hz}, 2 \mathrm{H}, \mathrm{H}-2^{\prime}$ and $\mathrm{H}-6$ ') 5.20 (brs, $2 \mathrm{H}, \mathrm{CH}_{2}-$ exocyclic), 2.89 (brs, 4H, $\mathrm{CH}_{2}$-pyrrolidyl), 1.71 (brs, 4H, $\mathrm{CH}_{2}$-pyrrolidyl). ${ }^{13} \mathrm{C}$ NMR $\left(75.5 \mathrm{MHz}\right.$, DMSO- $\left.d_{6}\right): \delta 142.8$ (C-1), 137.2 (C-3), 113.9 (C-4), 120.9 (C-4b), 122.4 (C-5), 120.5 (C-6), 129.5 (C-7), 112.8 (C-8), 141.6 (C-8a), 134.0 (C-9a), 131.1 (C-1'), 128.6 (C-2'/6'), 128.9 (C-3'/5'), 129.2 (C-4'), 177.8 (C-2”), 159.5 (C-5”), $66.1\left(\mathrm{CH}_{2}^{-}\right.$ exocyclic), $49.5\left(\mathrm{CH}_{2}\right.$-pyrrolidyl), $23.6\left(\mathrm{CH}_{2}\right.$-pyrrolidyl $)$. EIMS, $70 \mathrm{eV}, \mathrm{m} / \mathrm{z}$ (rel. int., \%): 427 (5, $\left.\mathrm{M}^{+}\right), 243$ (100), 189 (10), 121 (40).

1-(3-Nitrophenyl)-3-[3-pyrrolidylamino(methyl)-2-thioxo1,3,4-oxadiazol-5-yl] $\beta$-carboline (6b)

Yield: $52 \%, \mathrm{mp} 112.0-114.0{ }^{\circ} \mathrm{C}$. IR (KBr) $v_{\max } / \mathrm{cm}^{-1}$ : $3285(\mathrm{~N}-\mathrm{H}), 1623(\mathrm{C}=\mathrm{N}), 1347$ and $1252(\mathrm{C}=\mathrm{S}), 1523$, 
1498 and $1455(\mathrm{C}=\mathrm{C}) .{ }^{1} \mathrm{H}$ NMR (300 MHz, DMSO- $\left.d_{6}\right)$ : $\delta 8.95$ (s, 1H, H-4), 8.54 (d, $J 7.8 \mathrm{~Hz}, 1 \mathrm{H}, \mathrm{H}-5), 7.37$ (t, $J$ $7.8 \mathrm{~Hz}, 1 \mathrm{H}, \mathrm{H}-6), 7.63-7.72$ (m, 2H, H-7 and H-8), 12.17 (s, $1 \mathrm{H}, 9-\mathrm{NH}), 8.80$ (s, $1 \mathrm{H}, \mathrm{H}-2$ '), $8.50(\mathrm{~d}, J 7.8 \mathrm{~Hz}, 1 \mathrm{H}$, H-4'), 7.96 (t, J 7.8 Hz, 1H, H-5'), 8.44 (d, J 7.8 Hz, 1H, H-6'), 5.20 (brs, $2 \mathrm{H}, \mathrm{CH}_{2}$-exocyclic), 2.89 (brs, $4 \mathrm{H}, \mathrm{CH}_{2}-$ pyrrolidyl), 1.71 (brs, $4 \mathrm{H}, \mathrm{CH}_{2}$-pyrrolidyl). ${ }^{13} \mathrm{C} \mathrm{NMR}(75.5$ MHz, DMSO- $d_{6}$ ): $\delta 141.7$ (C-1), 138.6 (C-3), 130.1 (C-4), 120.9 (C-4b), 122.5 (C-5), 120.7 (C-6), 129.3 (C-7), 112.7 (C-8), 140.2 (C-8a), 134.1 (C-9a), 131.4 (C-1'), 123.4 (C-2'), 148.2 (C-3'), 123.7 (C-4'), 130.6 (C-5'), 135.1 (C-6'), 177.9 (C-2"), 159.3 (C-5"), 66.2 ( $\mathrm{CH}_{2}$-exocyclic), $49.5\left(\mathrm{CH}_{2}\right.$-pyrrolidyl), $23.6\left(\mathrm{CH}_{2}\right.$-pyrrolidyl). EIMS, $70 \mathrm{eV}$, $\mathrm{m} / \mathrm{z}$ (rel. int., \%): $472\left(10, \mathrm{M}^{+}\right), 289$ (70), 188 (10), 121 (25), 70 (100).

1-(4-N,N-Dimethylaminophenyl)-3-[3-pyrrolidylamino (methyl)-2-thioxo-1,3,4-oxadiazol-5-yl] $\beta$-carboline (6c)

Yield: $92 \%, \mathrm{mp} 156.0-160.0{ }^{\circ} \mathrm{C}$. IR $(\mathrm{KBr}) v_{\max } / \mathrm{cm}^{-1}$ : $3080(\mathrm{~N}-\mathrm{H}), 1623(\mathrm{C}=\mathrm{N}), 1369$ and $1243(\mathrm{C}=\mathrm{S}), 1557,1497$ and $1455(\mathrm{C}=\mathrm{C}) .{ }^{1} \mathrm{H}$ NMR $\left(300 \mathrm{MHz}, \mathrm{DMSO}-d_{6}\right)$ : $\delta 8.74$ (s, 1H, H-4), 8.46 (d, J $8.1 \mathrm{~Hz}, 1 \mathrm{H}, \mathrm{H}-5), 7.32$ (t, J $8.1 \mathrm{~Hz}$, $1 \mathrm{H}, \mathrm{H}-6), 7.60$ (t, $J 8.1 \mathrm{~Hz}, 1 \mathrm{H}, \mathrm{H}-7), 7.71$ (d, $J 8.1,1 \mathrm{H}$, H-8), 11.87 (s, 1H, 9-NH), 7.96 (d, J 9.0 Hz, 1H, H-2'), 6.97 (d, J 9.0 Hz, 1H, H-3'), 6.96 (d, J 9.0 Hz, 1H, H-5'), 7.96 (d, J 9.0 Hz, 1H, H-6'), 3.05 (s, 1H, N( $\left.\left.\mathrm{CH}_{3}\right)_{2}\right), 5.20$ (brs, $2 \mathrm{H}, \mathrm{CH}_{2}$-exocyclic), 2.89 (brs, $4 \mathrm{H}, \mathrm{CH}_{2}$-pyrrolidyl), 1.71 (brs, $4 \mathrm{H}, \mathrm{CH}_{2}$-pyrrolidyl). ${ }^{13} \mathrm{C}$ NMR $(75.5 \mathrm{MHz}$, DMSO- $\left.d_{6}\right): \delta 143.5$ (C-1), $133.5(\mathrm{C}-3), 112.7$ (C-4), 124.6 (C-4a), 121.0 (C-4b), 122.1 (C-5), 120.3 (C-6), 128.6 (C7), 112.8 (C-8), 141.4 (C-8a), 130.8 (C-9a), 129.0 (C-1'), 129.4 (C-2'), 112.1 (C-3'), 150.9 (C-4'), 112.1 (C-5'), 129.4 (C-6'), 177.6 (C-2”), 163.7 (C-5"), $40.0-\mathrm{N}\left(\mathrm{CH}_{3}\right)_{2}$, $66.1\left(\mathrm{CH}_{2}\right.$-exocyclic), $49.5\left(\mathrm{CH}_{2}\right.$-pyrrolidyl $), 23.6\left(\mathrm{CH}_{2}-\right.$ pyrrolidyl). EIMS, $70 \mathrm{eV}, \mathrm{m} / \mathrm{z}$ (rel. int., \%): $470\left(10, \mathrm{M}^{+}\right)$, 287 (100), 120 (35).

\section{1-Phenyl-3-[3-morpholylamino(methyl)-2-thioxo-1,3,4- oxadiazol-5-yl] $\beta$-carboline (7a)}

Yield: 82\%, mp 208.0-210.0 ${ }^{\circ} \mathrm{C}$. IR (KBr) $v_{\max } / \mathrm{cm}^{-1}$ : $3207(\mathrm{~N}-\mathrm{H}$ indol), $1622(\mathrm{C}=\mathrm{N}), 1359$ and $1248(\mathrm{C}=\mathrm{S})$, 1561, 1496 and $1455 \mathrm{~cm}^{-1}(\mathrm{C}=\mathrm{C}) .{ }^{1} \mathrm{H}$ NMR $(300 \mathrm{MHz}$, DMSO- $\left.d_{6}\right): \delta 8.90(\mathrm{~s}, 1 \mathrm{H}, \mathrm{H}-4), 8.52(\mathrm{~d}, J 7.8 \mathrm{~Hz}, 1 \mathrm{H}$, H-5), 7.35 (t, J 7.8, 1H, H-6), 7.58-7.73 (m, 5H, H-7, H-8, H-3', H-4' and H-5'), 12.03 (s, 1H, 9-NH), 8.05 (d, J 6.9 $\mathrm{Hz}, 1 \mathrm{H}, \mathrm{H}-2^{\prime}$ and $\mathrm{H}-6$ '), 5.08 (brs, $2 \mathrm{H}, \mathrm{CH}_{2}$-exocyclic), 2.81 (t, $J 4.3 \mathrm{~Hz}, 4 \mathrm{H}, \mathrm{CH}_{2}$-morpholyl), 3.61 (t, $J 4.3 \mathrm{~Hz}$, $4 \mathrm{H}, \mathrm{CH}_{2}$-morpholyl). ${ }^{13} \mathrm{C}$ NMR (75.5 MHz, DMSO- $\left.d_{6}\right): \delta$ 143.0 (C-1), 137.2 (C-3), 114.3 (C-4), 129.0 (C-4a), 120.9 (C-4b), 122.4 (C-5), 120.5 (C-6), 129.5 (C-7), 112.8 (C-8), 141.6 (C-8a), 134.1 (C-9a), 130.6 (C-1'), 128.6 (C-2'/6'),
128.9 (C-3'/5'), 129.3 (C-4'), 177.6 (C-2”), 161.2 (C-5”), $70.0\left(\mathrm{CH}_{2}\right.$-exocyclic), $50.1\left(\mathrm{CH}_{2}\right.$-morpholyl), $66.4\left(\mathrm{CH}_{2}-\right.$ morpholyl). EIMS, $70 \mathrm{eV}, \mathrm{m} / z$ (rel. int., \%): $443\left(5, \mathrm{M}^{+}\right)$, 243 (100), 121 (20), 58 (25).

1-(3-Nitrophenyl)-3-[3-morpholylamino(methyl)-2-thioxo1,3,4-oxadiazol-5-yl] $\beta$-carboline (7b)

Yield: $85 \%$, mp 163.0-167.0 ${ }^{\circ} \mathrm{C}$. IR (KBr) $v_{\max } / \mathrm{cm}^{-1}$ : $3226(\mathrm{~N}-\mathrm{H}), 1626(\mathrm{C}=\mathrm{N}), 1351$ and $1249(\mathrm{C}=\mathrm{S}), 1559$, 1497 and $1453(\mathrm{C}=\mathrm{C}) .{ }^{1} \mathrm{H}$ NMR (300 MHz, DMSO- $\left.d_{6}\right)$ : $\delta$ 8.98 (s, 1H, H-4), 8.55 (d, J 7.8 Hz, 1H, H-5), 7.38 (t, J 7.8 Hz, 1H, H-6), 7.64-7.73 (m, 2H, H-7, H-8), 12.21 (s, 1H, 9-NH), 8.80 (s, 1H, H-2'), 8.50 (d, J 7.8 Hz, 1H, H-4'), 7.97 (t, $J 7.8 \mathrm{~Hz}, 1 \mathrm{H}, \mathrm{H}-5$ '), 8.45 (dd, $J 7.8$ and $1.8 \mathrm{~Hz}, 2 \mathrm{H}$, H-6'), 5.09 (brs, $2 \mathrm{H}, \mathrm{CH}_{2}$-exocyclic), 2.81 (brs, $4 \mathrm{H}, \mathrm{CH}_{2}$ morpholyl), 3.61 (brs, $\mathrm{CH}_{2}$-morpholyl). ${ }^{13} \mathrm{C}$ NMR (75.5 MHz, DMSO- $\left.d_{6}\right): \delta 141.7$ (C-1), 148.2 (C-3), 115.0 (C-4), 122.6 (C-5), 120.8 (C-6), 129.4 (C-7), 112.7 (C-8), 140.4 (C-8a), 134.3 (C-9a), 130.1 (C-1'), 123.4 (C-2'), 148.2 (C3'), 123.8 (C-4'), 130.6 (C-5'), 135.1 (C-6'), 177.7 (C-2'), 160.6 (C-5”), $70.0\left(\mathrm{CH}_{2}\right.$-exocyclic), $50.1\left(\mathrm{CH}_{2}\right.$-morpholyl), $66.0\left(\mathrm{CH}_{2}\right.$-morpholyl).EIMS, $70 \mathrm{eV}, \mathrm{m} / \mathrm{z}$ (rel. int., \%): 488 $\left(5, \mathrm{M}^{+}\right), 289$ (95), 242 (100), 121 (20), 57 (25).

\section{1-(4-N,N-Dimethylaminophenyl)-3-[3-morpholylamino} (methyl)-2-thioxo-1,3,4-oxadiazol-5-yl] $\beta$-carboline (7c)

Yield: $83 \%, \mathrm{mp} 215.0-217.0{ }^{\circ} \mathrm{C}$. IR $(\mathrm{KBr}) \mathrm{v}_{\max } / \mathrm{cm}^{-1}$ : $3261(\mathrm{~N}-\mathrm{H}), 1609(\mathrm{C}=\mathrm{N}), 1364$ and $1238(\mathrm{C}=\mathrm{S}), 1556$, 1495 and $1438(\mathrm{C}=\mathrm{C}) .{ }^{1} \mathrm{H}$ NMR (300 MHz, DMSO- $d_{6}$ ): $\delta 8.75$ (s, 1H, H-4), 8.46 (d, J 8.1 Hz, 1H, H-5), 7.33 (t, $J 8.1 \mathrm{~Hz}, 1 \mathrm{H}, \mathrm{H}-6), 7.60$ (t, J 8.1 Hz, H-7), 7.72 (d, J 8.1 $\mathrm{Hz}, 1 \mathrm{H}, \mathrm{H}-8), 11.90$ (s, 1H, 9-NH), 7.96 (d, J $8.7 \mathrm{~Hz}$, 1H, H-2'), 6.97 (d, J $8.7 \mathrm{~Hz}, 1 \mathrm{H}, \mathrm{H}-3$ '), 6.97 (d, J 8.7 Hz, 1H, H-5'), 7.96 (d, J 8.7 Hz, 1H, H-6'), 3.05 (s, 6H, $\left.\mathrm{N}\left(\mathrm{CH}_{3}\right)_{2}\right), 5.08$ (brs, $2 \mathrm{H}, \mathrm{CH}_{2}$-exocyclic), 2.81 (brs, $4 \mathrm{H}$, $\mathrm{CH}_{2}$-morpholyl), 3.61 (brs, $\mathrm{CH}_{2}$-morpholyl). ${ }^{13} \mathrm{C}$ NMR $\left(75.5 \mathrm{MHz}, \mathrm{DMSO}-d_{6}\right): \delta 143.6(\mathrm{C}-1), 133.6(\mathrm{C}-3), 112.9$ (C-4), 124.5 (C-4a), 121.0 (C-4b), 122.1 (C-5), 120.4 (C-6), 128.6 (C-7), 112.8 (C-8), 141.4 (C-8a), 130.7 (C-9a), 128.9 (C-1'), 129.4 (C-2'), 112.1 (C-3'), 150.9 (C-4'), 112.1 (C-5'), 129.4 (C-6'), 177.6 (C-2”), 161.4 (C-5"), $39.9-\mathrm{N}\left(\mathrm{CH}_{3}\right)_{2}, 69.9\left(\mathrm{CH}_{2}\right.$-exocyclic), $50.1\left(\mathrm{CH}_{2}-\right.$ morpholyl), $66.0\left(\mathrm{CH}_{2}\right.$-morpholyl). EIMS, $70 \mathrm{eV}, \mathrm{m} / \mathrm{z}$ (rel. int., \%): $486\left(5, \mathrm{M}^{+}\right), 242$ (30), 121 (15), 57 (100).

\section{Anticancer assays}

The synthesized compounds were evaluated in vitro against eight-cell panel lines consisting of melanoma (UACC-62), breast (MCF7), ovarian resistant (NCI/ADR), renal (786-0), lung (NCI-460), prostate (PCO-3), ovarian 
(OVCAR) and colon (HT-29). The tests were performed by the colorimetric method with sulforodamine $\mathrm{B}$, according to NCI standard protocol and doxorubicin was used as positive control. ${ }^{22}$ Assays were performed in a 96-well plate using four concentrations at 10 -fold dilutions $\left(0.25 \mathrm{mg} \mathrm{mL}^{-1}\right.$ to $250 \mathrm{mg} \mathrm{mL}^{-1}$ ) for each test compound. The anticancer activity was deduced from concentration-response curves and three concentration response parameters $\left(\mathrm{GI}_{50}\right.$, TGI and $\mathrm{LC}_{50}$ ) were calculated. The $\mathrm{GI}_{50}$ values (growth inhibitory activity) (Table 1) refer to the drug concentration that produce $50 \%$ reduction of cellular growth when compared to untreated control cells. ${ }^{23}$ The TGI (cytostatic activity) and $\mathrm{LC}_{50}$ (cytotoxic activity) values parameters (Table 2) refer to the drug concentration for total growth inhibition and for killing $50 \%$ of cells, respectively. Compounds with $\mathrm{GI}_{50}$ values $<100 \mu \mathrm{mol} \mathrm{L}{ }^{-1}$ were considered active. A mean graph midpoint values (MG-MID) of the compounds toward tumor cell lines were calculated for each of the parameters $\mathrm{GI}_{50}$, TGI and $\mathrm{LC}_{50}$. MG-MID values supplied an averaged activity parameter for all cell lines. ${ }^{23}$

\section{Antimicrobial activity}

The bacterial strains were grown overnight at $36{ }^{\circ} \mathrm{C}$ in Nutrient Agar (Merck), and the strains were grown in Saboraud Dextrose Agar. Inoculum for the assays was prepared by diluting scraped cell mass in $0.85 \% \mathrm{NaCl}$ solution, adjusted to McFarland scale 0.5 and confirmed by spectrophotometrical reading at $580 \mathrm{~nm}$. Cell suspensions were finally diluted to $10^{4} \mathrm{UFC} \mathrm{mL}^{-1}$ for use in the activity assays. Minimal Inhibitory Concentration (MIC) tests were carried out using Müller-Hinton broth (bacteria) or RPMI1640 (yeasts) on a tissue culture test plate (96 wells). ${ }^{24}$ Each compound was tested in duplicate. The stock solutions of compounds firstly in DMSO and subsequently in Tween 80 water solution $(0.1 \%)$ were diluted and transferred into the first well, and serial dilutions were performed so that concentrations in the range of $250-1.6 \mu \mathrm{g} \mathrm{mL}^{-1}$ were obtained. Chloramphenicol and nystatin (Merck) were used as the reference antibiotic control. The inoculum was added to all wells and the plates were incubated at $36^{\circ} \mathrm{C}$ for $24 \mathrm{~h}$. Antibacterial activity was detected by adding $20 \mu \mathrm{L}$ of $0.5 \%$ TTC (triphenyl tetrazolium chloride, Merck) aqueous solution. MIC was defined as the lowest concentration of the compounds that inhibited visible growth, as indicated by TTC staining (dead cells are not stained by TTC). For antifungal activity evaluation, after the incubation period changes in the RPMI-1640 medium color were verified from pink (original color) to yellow. The change indicates an acidification from medium by the microorganisms' growth. ${ }^{25}$

\section{Acknowledgments}

This work was supported by Conselho Nacional de Desenvolvimento Científico e Tecnológico (CNPq, Brazil) and Fundação de Amparo a Pesquisa de São Paulo (FAPESP). We thank CAPES and CNPq for fellowships (FCS, ASNF, VAB, JEC and MAF).

\section{Supplementary Information}

Supplementary information is available free of charge at http://jbcs.sbq.org.br, as PDF file.

\section{References}

1. Cao, R.; Peng, W.; Wang, Z.; Xu, A.; Curr. Med. Chem. 2007, 14,479 .

2. Cao, R.; Yi, W.; Wu,Q.; Guan, X.; Feng, M.; Ma, C.; Chen, Z.; Song, H.; Peng, W.; Bioorg. Med. Chem. Lett. 2008, 18, 6558.

3. Cao, R.; Peng, W.; Chen, H.; Hou, X.; Guan, H.; Chen, Q.; Ma, Y.; Xu, A.; Eur. J. Med. Chem. 2005, 40, 249.

4. Cao, R.; Chen, H.; Peng, W.; Ma, Y.; Hou, X.; Guan, H.; Liu, X.; Xu, A.; Eur. J. Med. Chem. 2005, 991.

5. Boursereau, Y.; Coldham, I.; Bioorg. Med. Chem. Lett. 2004, $14,5841$.

6. Zhao, M.; Bi, L.; Wang, W.; Wang, C.; Baudy-Floc'h, M.; Ju, J.; Peng, S.; Bioorg. Med. Chem. 2006, 14, 6998.

7. Guan, H.; Chen, H.; Peng, W.; Ma, Y.; Cao, R.; Liu, X.; Xu, A.; Eur. J. Med. Chem. 2006, 41, 1167.

8. Xiao, S.; Lin, W.; Wang, C.; Yang, M.; Bioorg. Med. Chem. Lett. 2001, 11, 437.

9. Cao, R.; Peng, W.; Chen, H.; Ma, Y.; Liu, X.; Hou, X.; Guan, H.; Xu, A.; Biochem. Biophys. Res. Commun. 2005, 338, 1557.

10. Deveau, A. M.; Labroli, M. A.; Dieckhaus, C. M.; Barthen, M. T.; Smith, K. S.; Macdonald, T. L.; Bioorg. Med. Chem. Lett. 2001, 11, 1251.

11. Schupp, P.; Poehner, T.; Edrada, R.; Ebel, R.; Berg, A.; Wray, V.; Proksch, P.; J. Nat. Prod. 2003, 66, 272.

12. Peng, J.; Hu, J.; Kazi, A.; Li, Z.; Avery, M.; Peraud, O.; Hill, R. T.; Franzblau, S. G.; Zhang, F.; Schinazi, R. F.; Wirtz, S. S.; Tharnish, P.; Kelly, M.; Wahyuono, S.; Hamann, M. T.; J. Am. Chem. Soc. 2003, 125, 13382.

13. Cabrera, G. M.; Seldes, A. M.; J. Nat. Prod. 1999, 62, 759.

14. Formagio, A. S. N.; Tonin, L.T. D.; Foglio, M. A.; Madjarof, C.; Carvalho, J. E. de; Costa, W. F. da; Cardoso, F. P.; Sarragiotto, M. H.; Bioorg. Med. Chem. 2008, 16, 9660.

15. Mamolo, M.G.; Zampieri, D.; Vio, L.; Fermeglia, M.; Ferone, M.; Pricl, S.; Scialino, G.; Banfi, E.; Bioorg. Med. Chem. 2005, 13, 3797.

16. Ali, M. A.; Shaharyar, M.; Bioorg. Med. Chem. Lett. 2007, 17, 3314. 
17. Aboraia, A. S.; Abdel-Rahman, H. M.; Mahfouz, N. M.; ELGendy, M. A.; Bioorg. Med. Chem. 2006, 14, 1236.

18. Lipinski, C.A.; Lombardo, F.; Dominy, B.W.; Feeney, P.J.; $A d v$. Drug Delivery Rev. 1997, 23, 3.

19. Zhao, M. Y. ; Abraham, M. H.; Le, J.; Hersey, A.; Luscombe, C. N.; Beck, G.; Sherborne, B.; Pharm. Res. 2002, 19, 1446.

20. http://www.molinspiration.com, accessed in September 2008.

21. www.organic-chemistry.org/prog/peo, accessed in September 2008.

22. Monks, A.; Scudiero, D.; SKehan, P.; Shoemaker, R.; Paull, K.; Vistica, D.; Hose, C.; Langley, J.; Cronise, P.; Vaigro-Wolff, A.; Gray-Goodrich, M.; Campbell, H.; Mayo, J.; Boyd, M.; J. Natl. Cancer Inst. 1991, 83, 757.
23. Holbeck, S.L.; Eur. J. Cancer 2004, 40, 785.

24. NCCLS; Methods for Dilution Antimicrobial Susceptibility Tests for Bacteria that Grow Aerobically, Approved Standard, $6^{\text {th }}$ ed., NCCLS document M7-A6: USA, 2003.

25. NCCLS; Methods for Broth Dilution Aantifungal Susceptibility Testing of Years, Approved Standard, $2^{\text {nd }}$ ed., NCCLS document M27-A2: USA, 2002.

Received: February 4, 2009

Web Release Date: November 19, 2009

FAPESP helped in meeting the publication costs of this article. 


\section{Synthesis, Antitumor and Antimicrobial Activity of Novel 1-Substituted} Phenyl-3-[3-alkylamino(methyl)-2-thioxo-1,3,4-oxadiazol-5-yl] $\beta$-Carboline Derivatives

Franciele C. Savariz, ${ }^{a}$ Anelise S. N. Formagio, ${ }^{a}$ Valéria A. Barbosa, ${ }^{a}$

Mary Ann Foglio, ${ }^{b}$ João E. de Carvalho, ${ }^{b}$ Marta C. T. Duarte, ${ }^{b}$

Benedito P. Dias Filho and Maria Helena Sarragiotto*,a

${ }^{a}$ Departamento de Química and ${ }^{c}$ Departamento de Análises Clínicas,

Universidade Estadual de Maringá, 87020-900 Maringá-PR, Brazil

${ }^{b}$ Centro Pluridisciplinar de Pesquisas Químicas, Biológicas e Agrícolas, Universidade Estadual de Campinas, CP 6171, 13083-970 Campinas-SP, Brazil

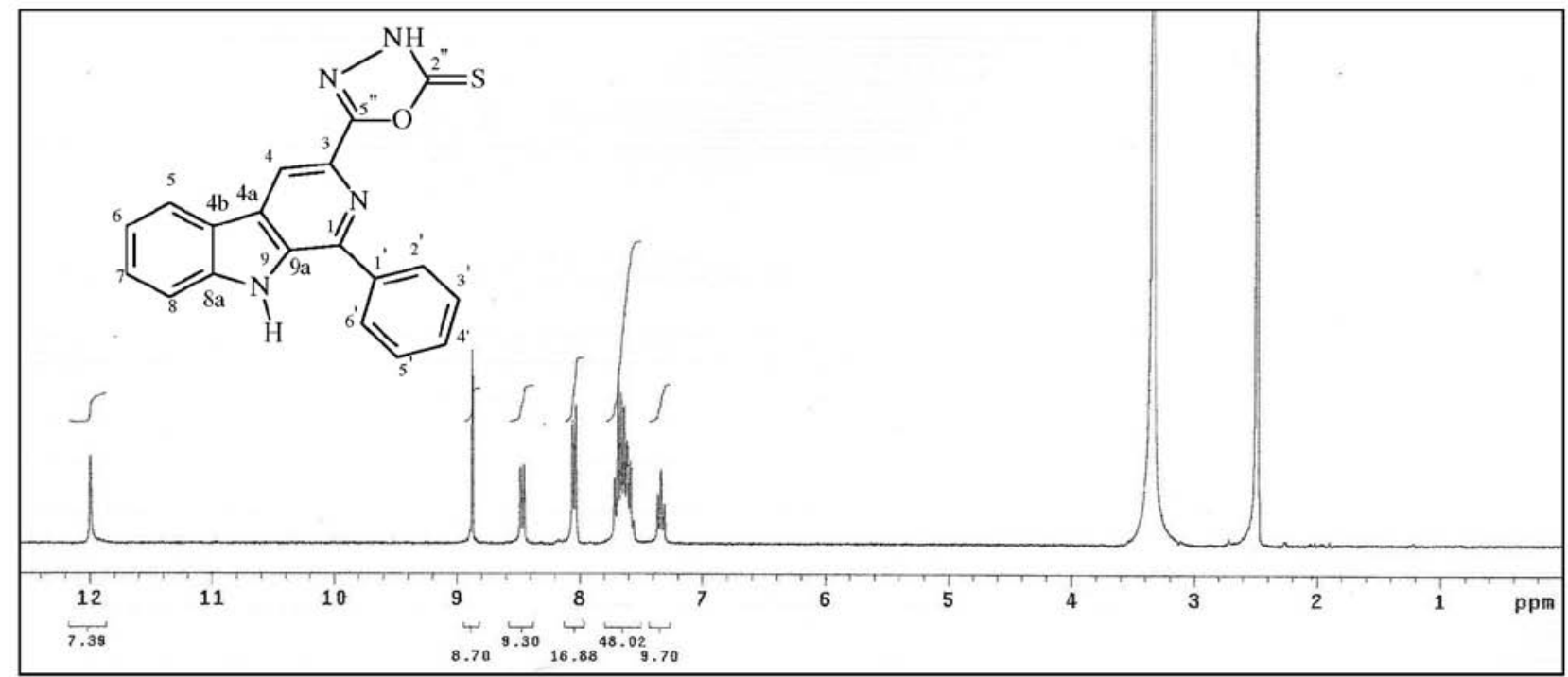

Figure S1. ${ }^{1} \mathrm{H}$ NMR spectra (300 MHz, DMSO- $d_{6}$ ) of compound 1 a. 


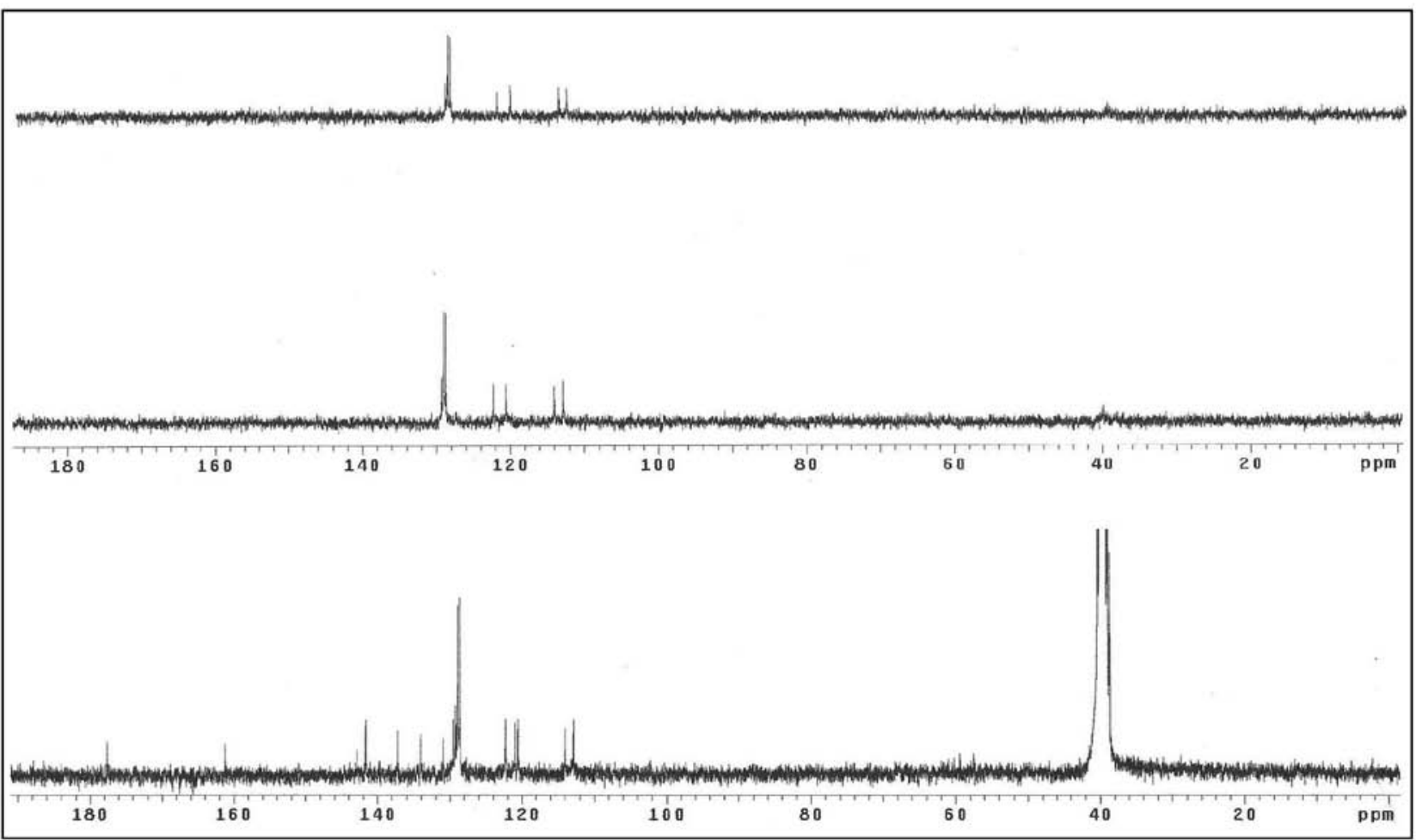

Figure S2. ${ }^{13} \mathrm{C}$ NMR / DEPT spectra (75.5 MHz, DMSO- $\left.d_{6}\right)$ of compound 1a.

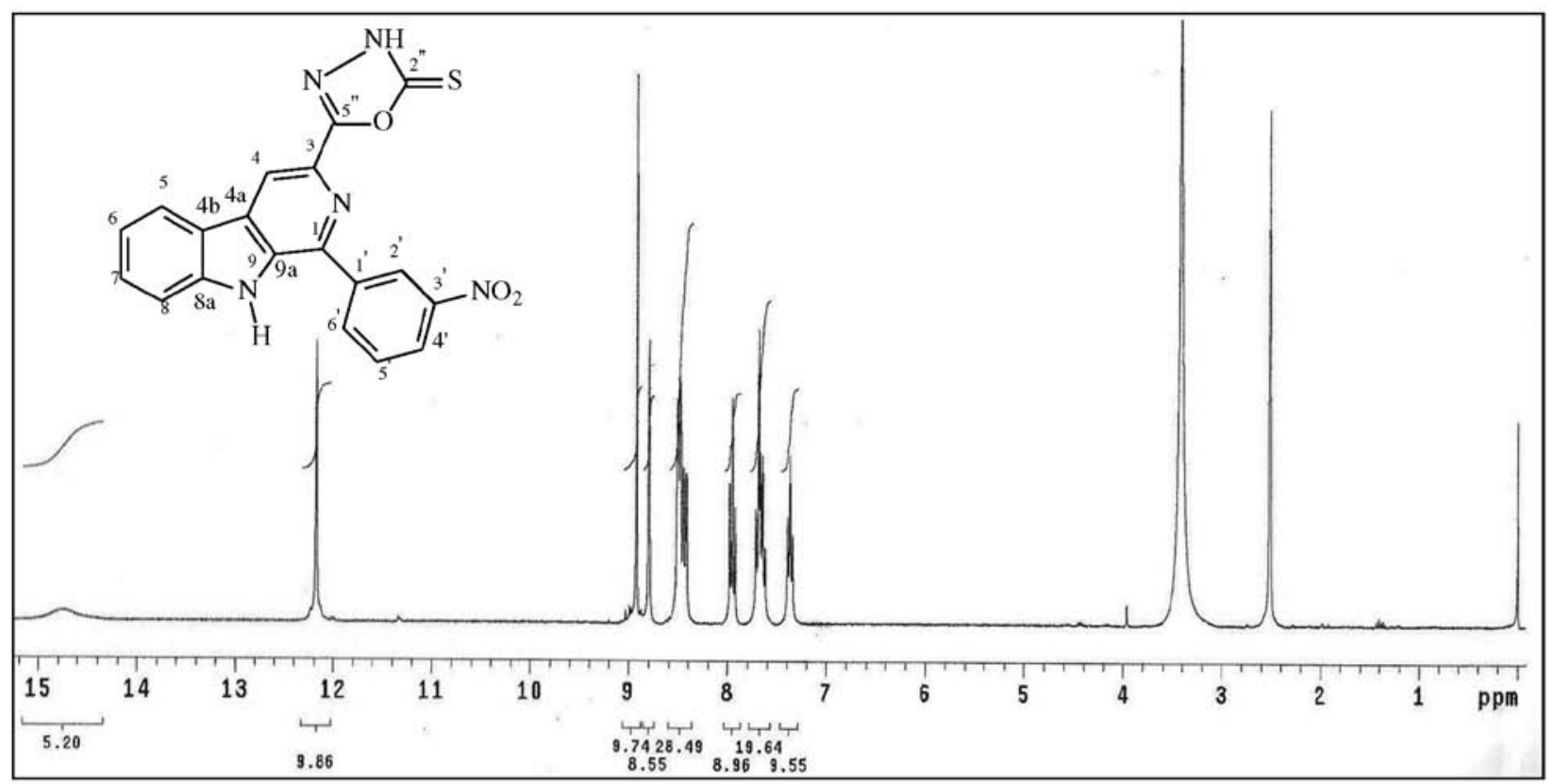

Figure S3. ${ }^{1} \mathrm{H}$ NMR spectra (300 MHz, DMSO- $d_{6}$ ) of compound $\mathbf{1 b}$. 


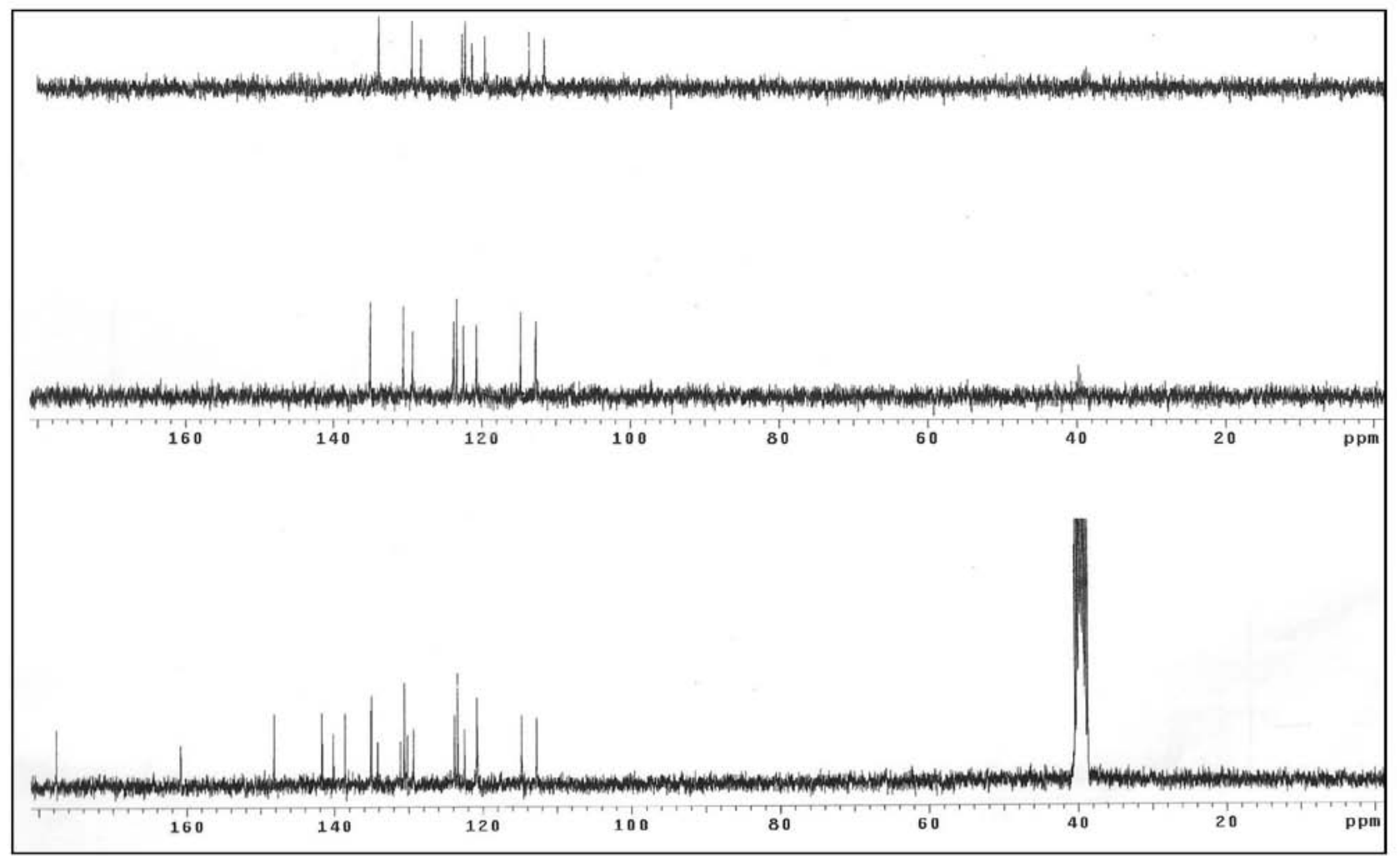

Figure S4. ${ }^{13} \mathrm{C}$ NMR / DEPT spectra (75.5 MHz, DMSO- $d_{6}$ ) of compound $\mathbf{1 b}$.

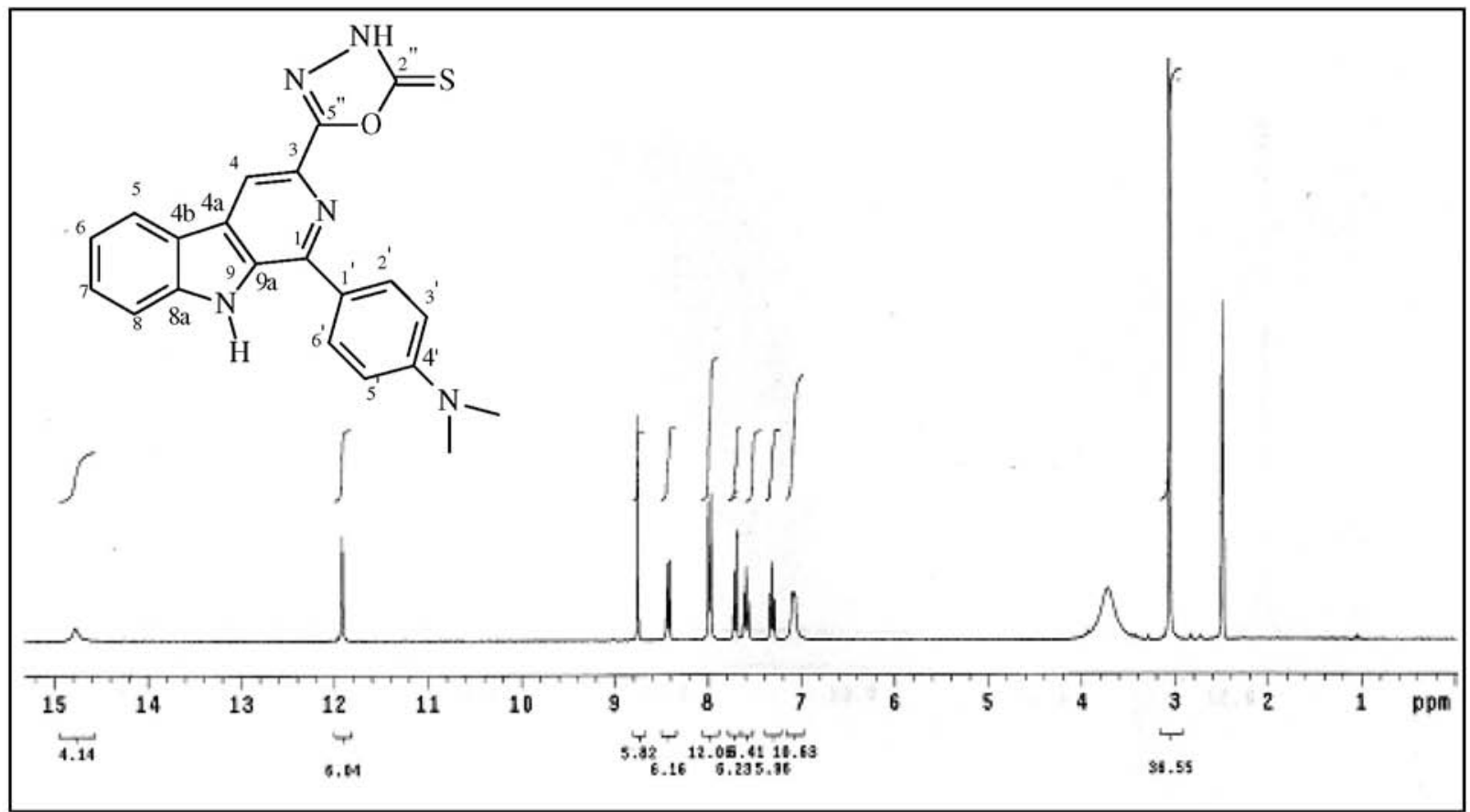

Figure S5. ${ }^{1} \mathrm{H}$ NMR spectra (300 MHz, DMSO- $d_{6}$ ) of compound 1c. 


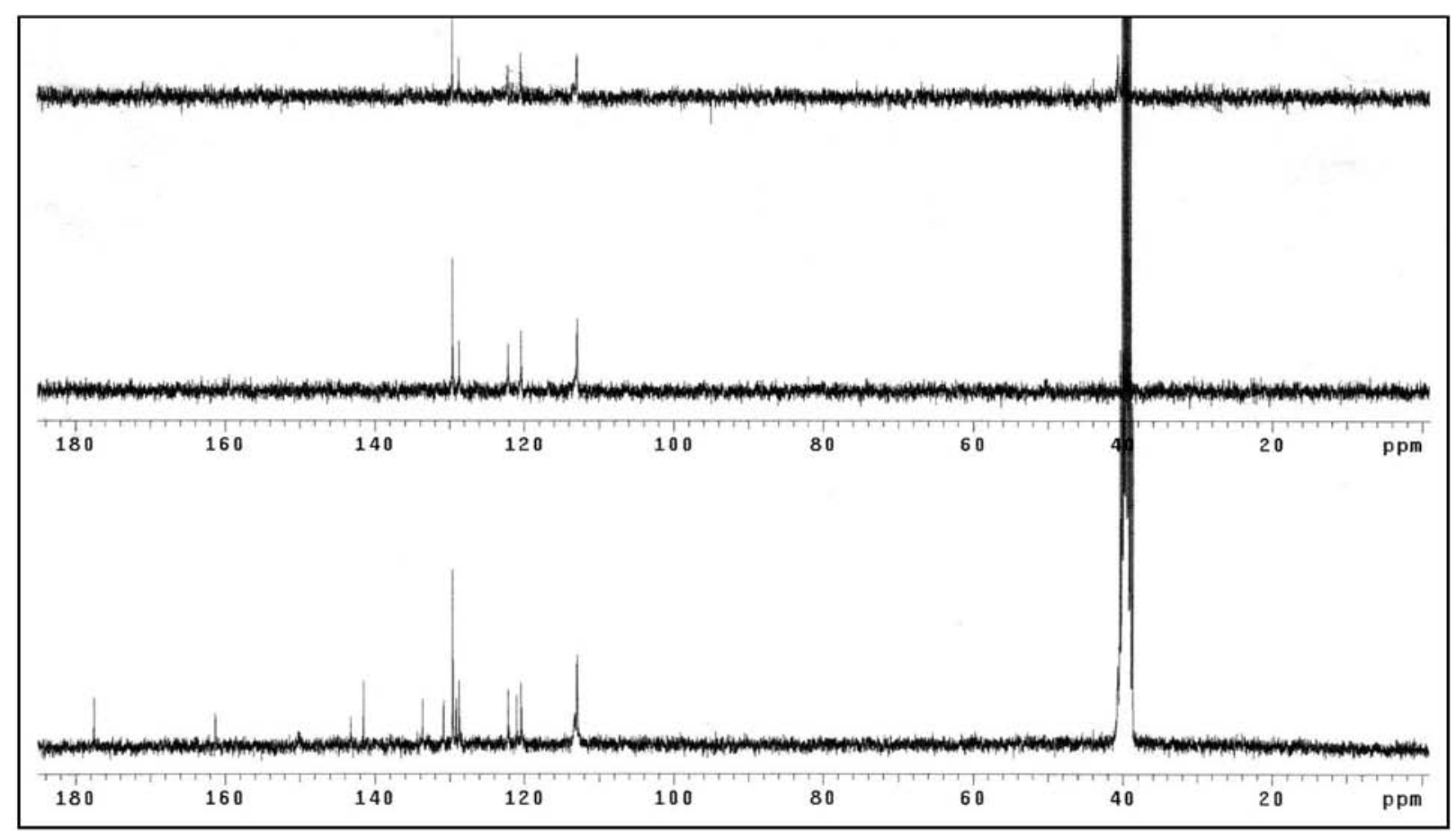

Figure S6. ${ }^{13} \mathrm{C}$ NMR / DEPT spectra $\left(75.5 \mathrm{MHz}\right.$, DMSO- $\left.d_{6}\right)$ of compound 1c.

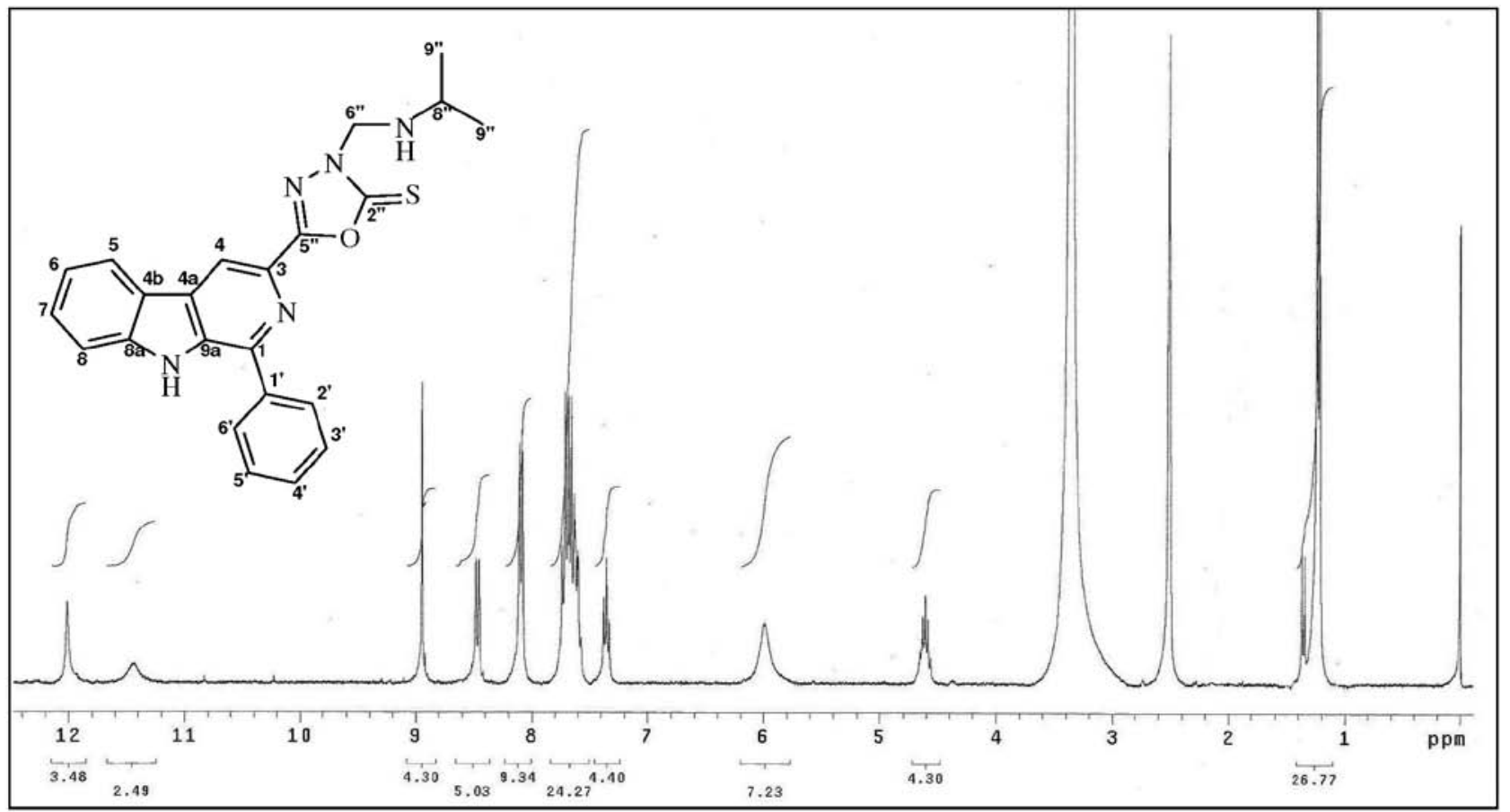

Figure S7. ${ }^{1} \mathrm{H}$ NMR spectra (300 MHz, DMSO- $d_{6}$ ) of compound 2a. 


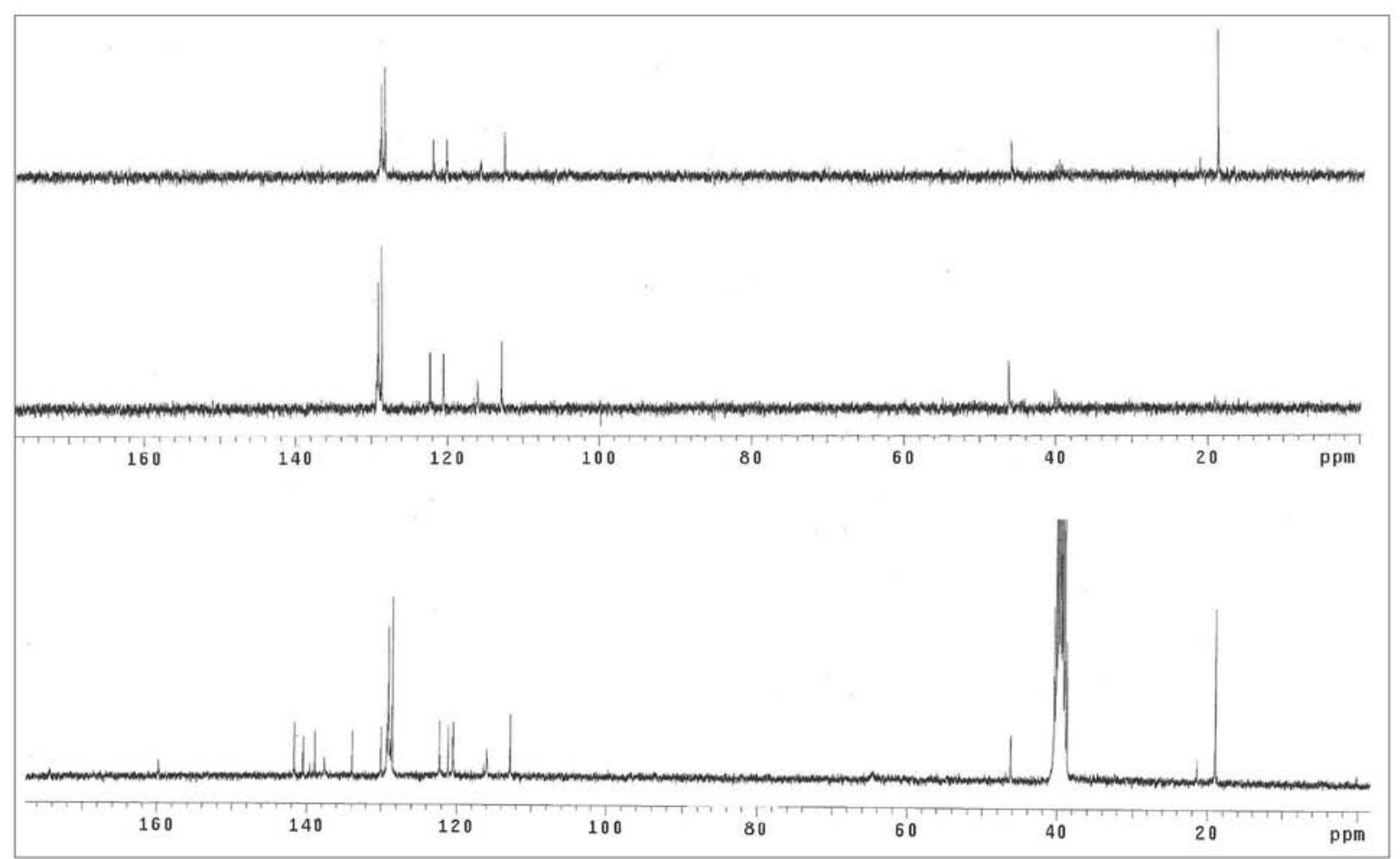

Figure S8. ${ }^{13} \mathrm{C}$ NMR / DEPT spectra (75.5 MHz, DMSO- $d_{6}$ ) of compound 2a.

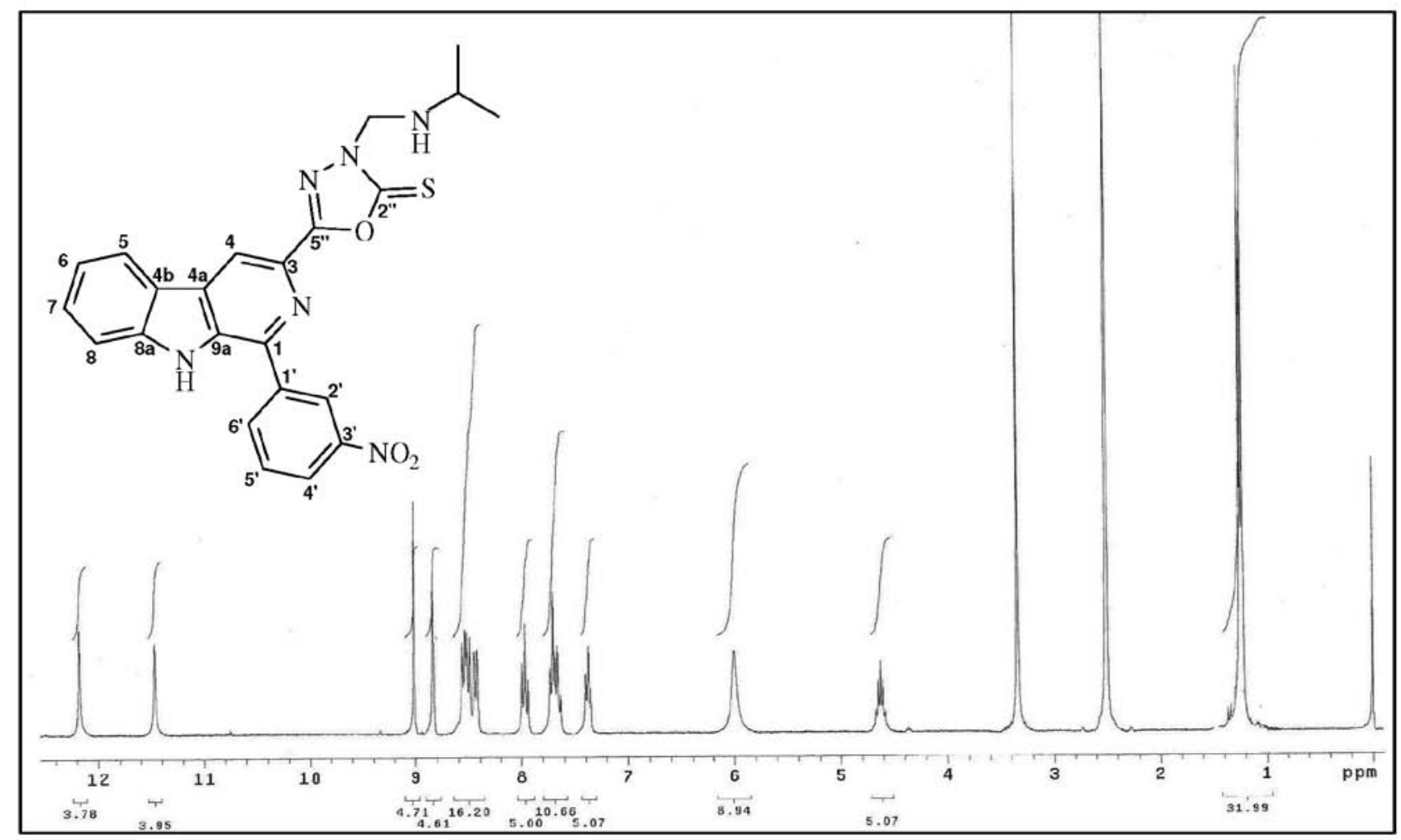

Figure S9. ${ }^{1} \mathrm{H}$ NMR spectra (300 MHz, DMSO- $d_{6}$ ) of compound $2 \mathbf{b}$. 


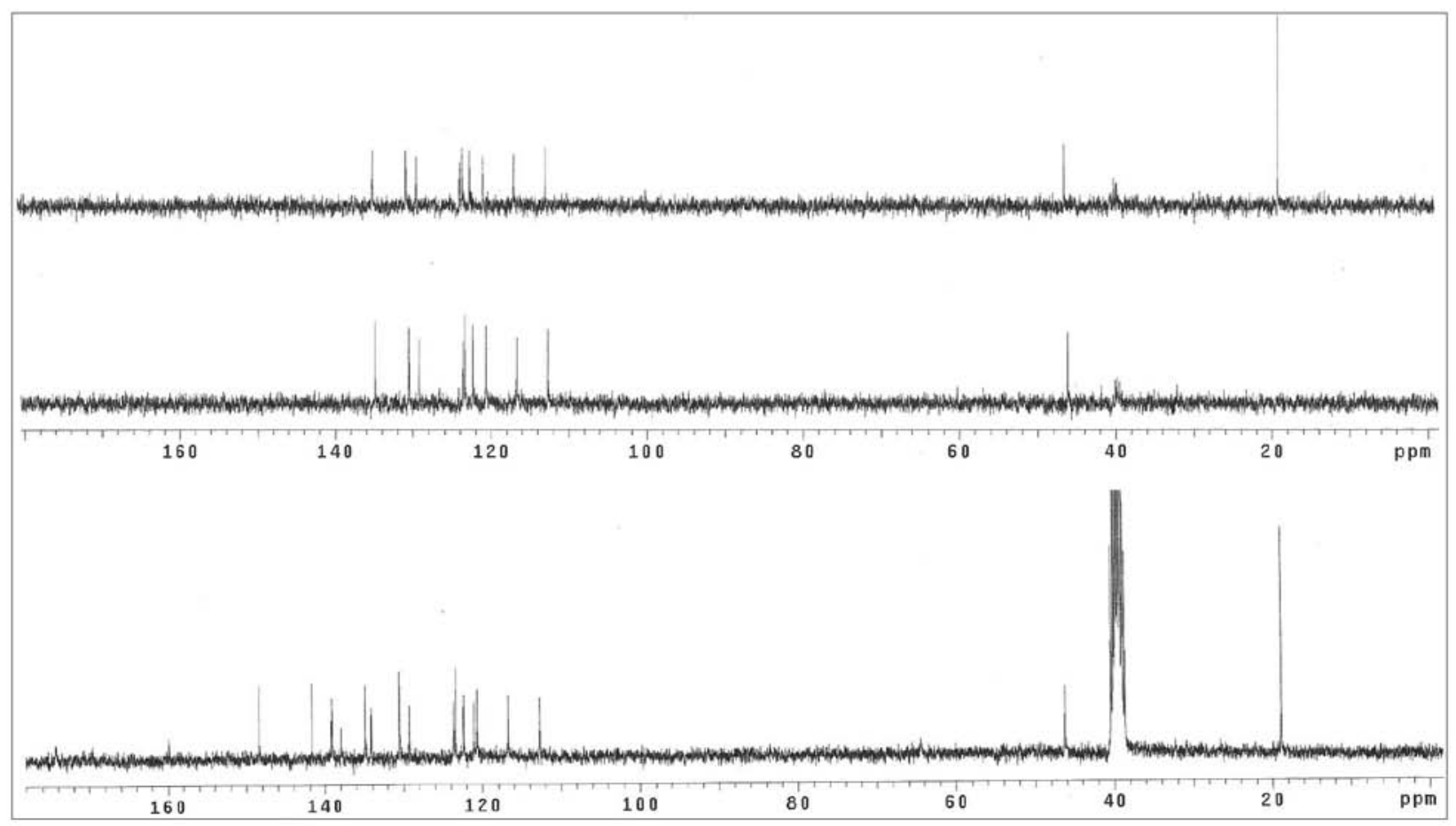

Figure S10. ${ }^{13} \mathrm{C}$ NMR / DEPT spectra (75.5 MHz, DMSO- $d_{6}$ ) of compound $2 \mathbf{b}$.

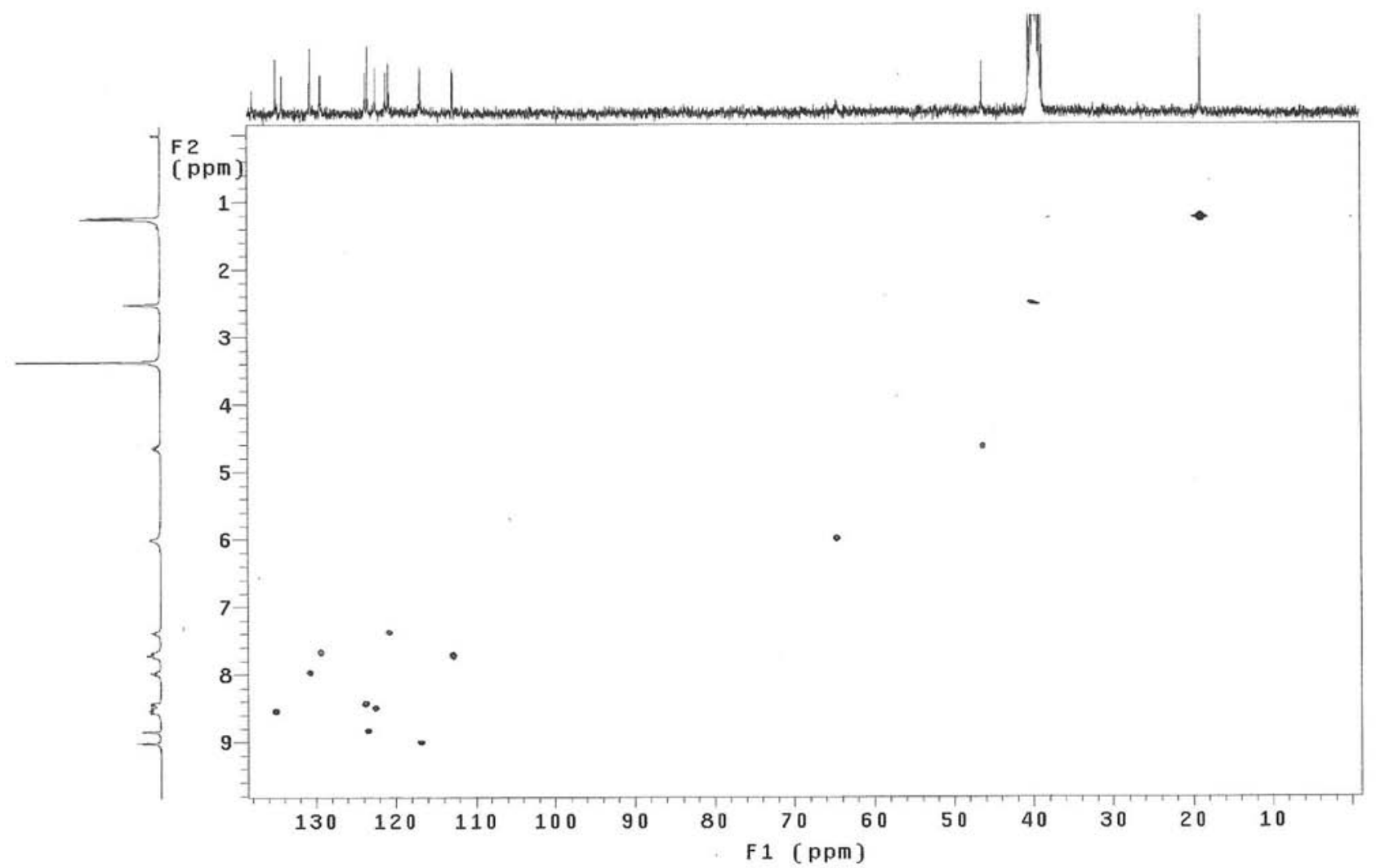

Figure S11. HMQC spectra of compound $\mathbf{2 b}$. 


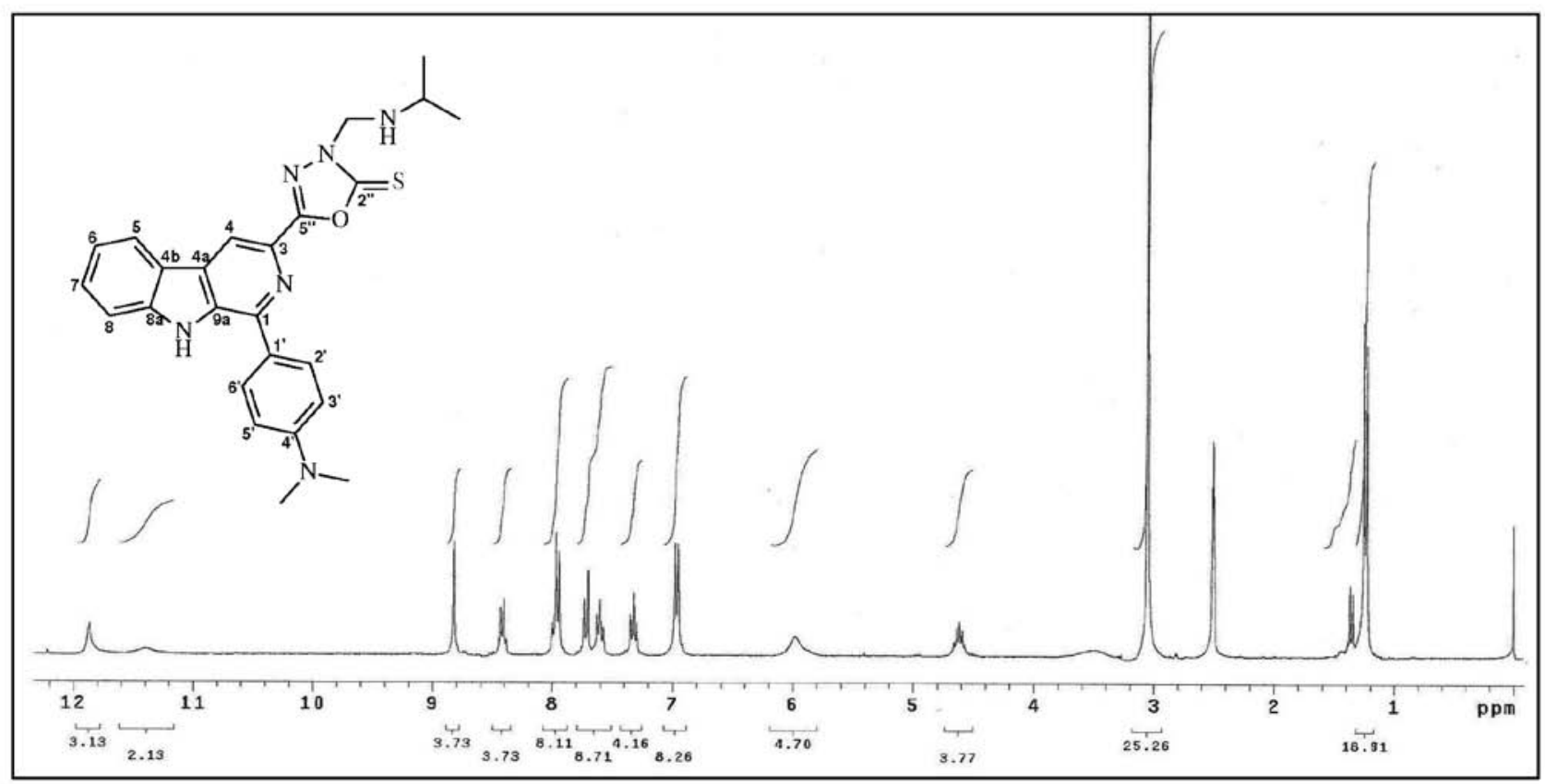

Figure S12. ${ }^{1} \mathrm{H}$ NMR spectra (300 MHz, DMSO- $d_{6}$ ) of compound 2c.

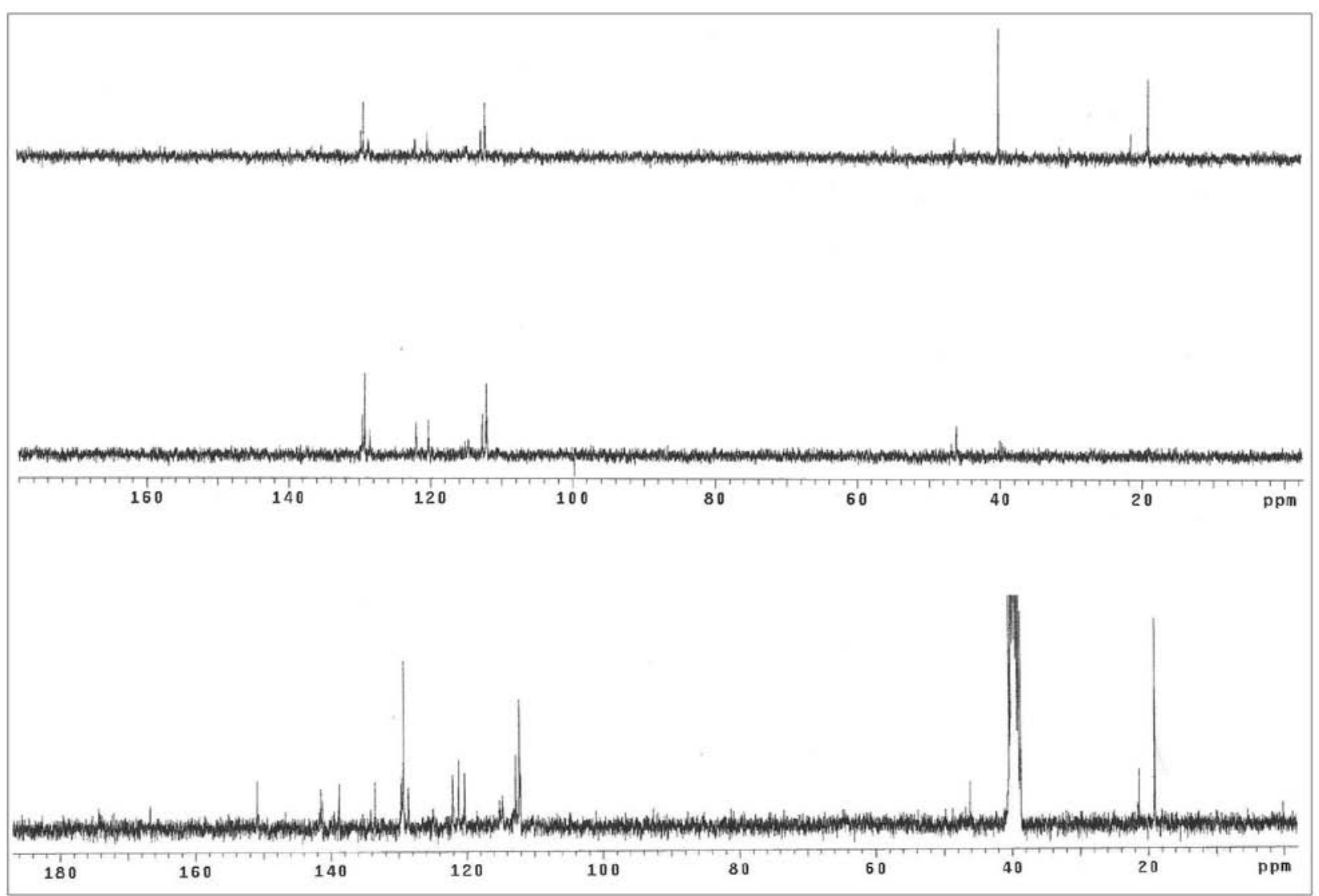

Figure S13. ${ }^{13} \mathrm{C}$ NMR / DEPT spectra (75.5 MHz, DMSO- $d_{6}$ ) of compound 2 c. 


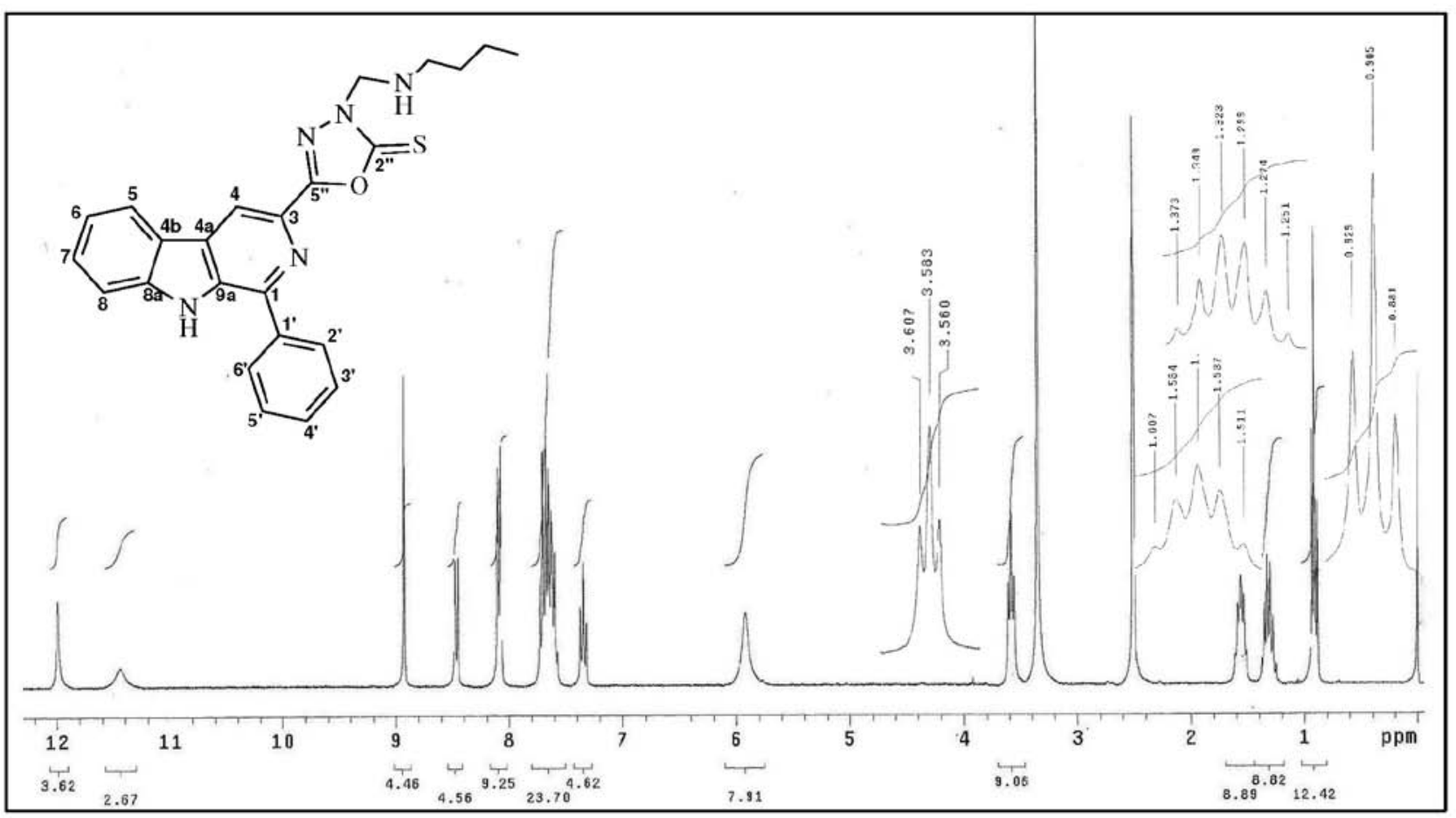

Figure S14. ${ }^{1} \mathrm{H}$ NMR spectra (300 MHz, DMSO- $d_{6}$ ) of compound 3a.

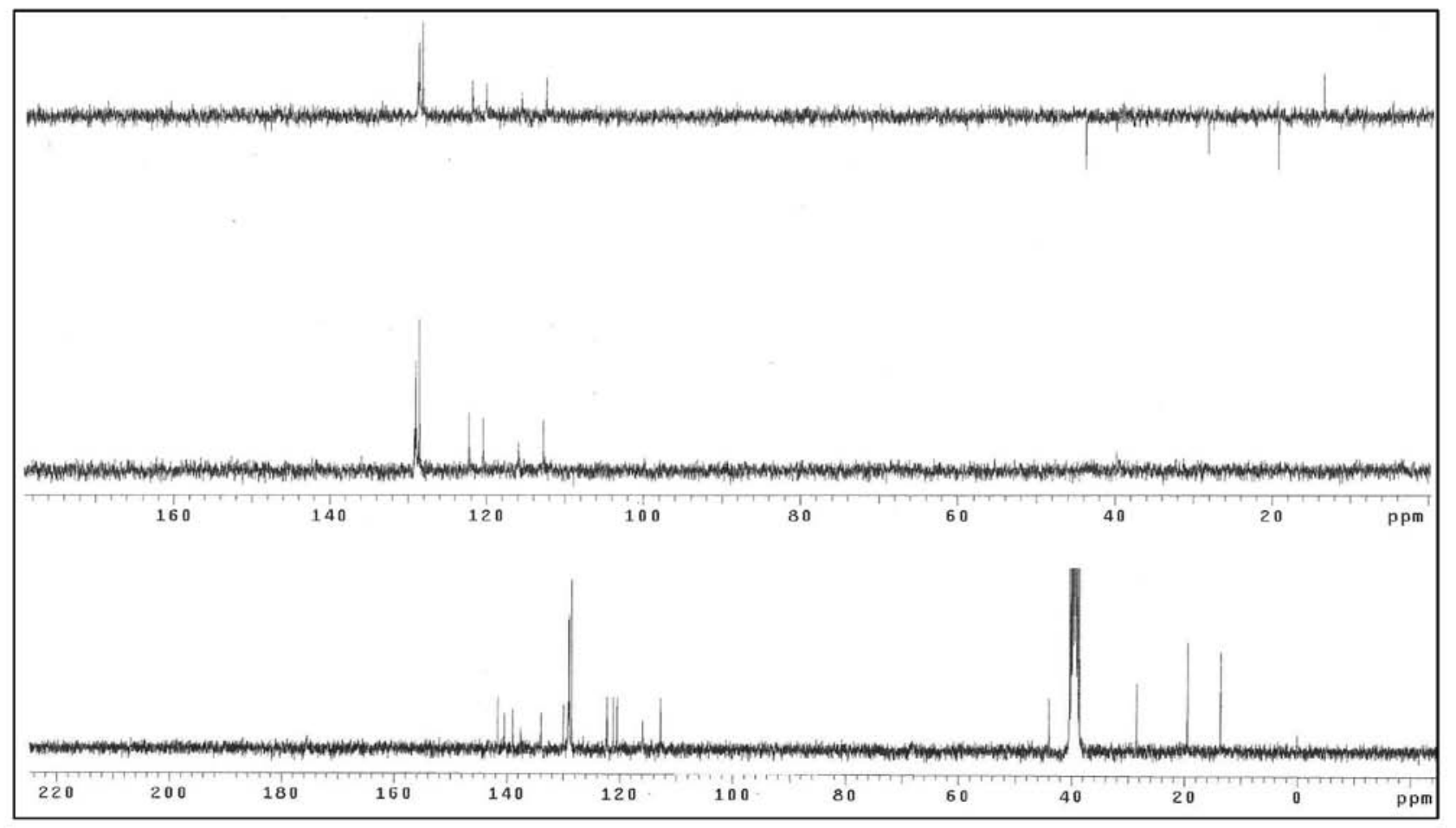

Figure S15. ${ }^{13} \mathrm{C}$ NMR / DEPT spectra (75.5 MHz, DMSO- $d_{6}$ ) of compound 3a. 


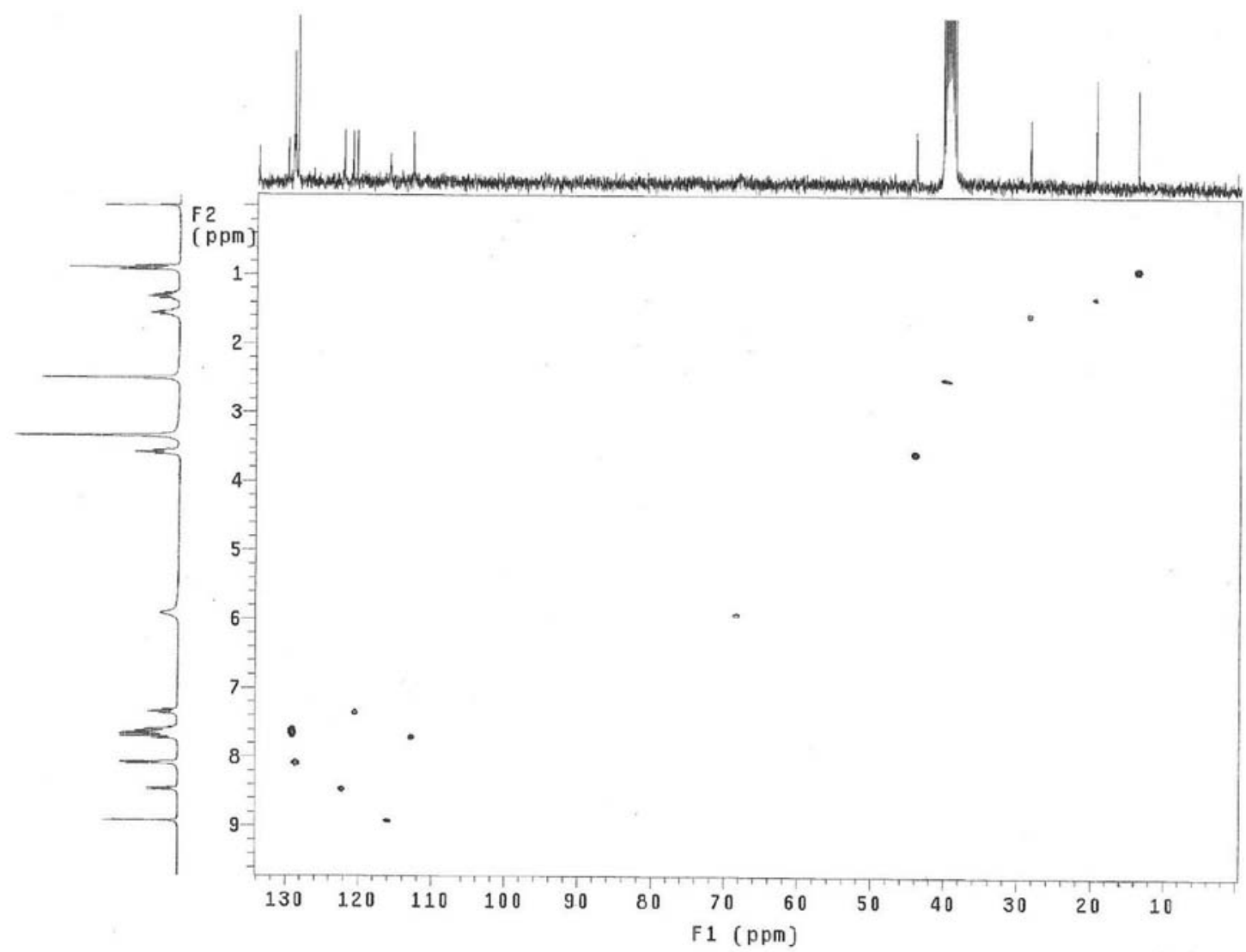

Figure S16. HMQC spectra of compound 3a.

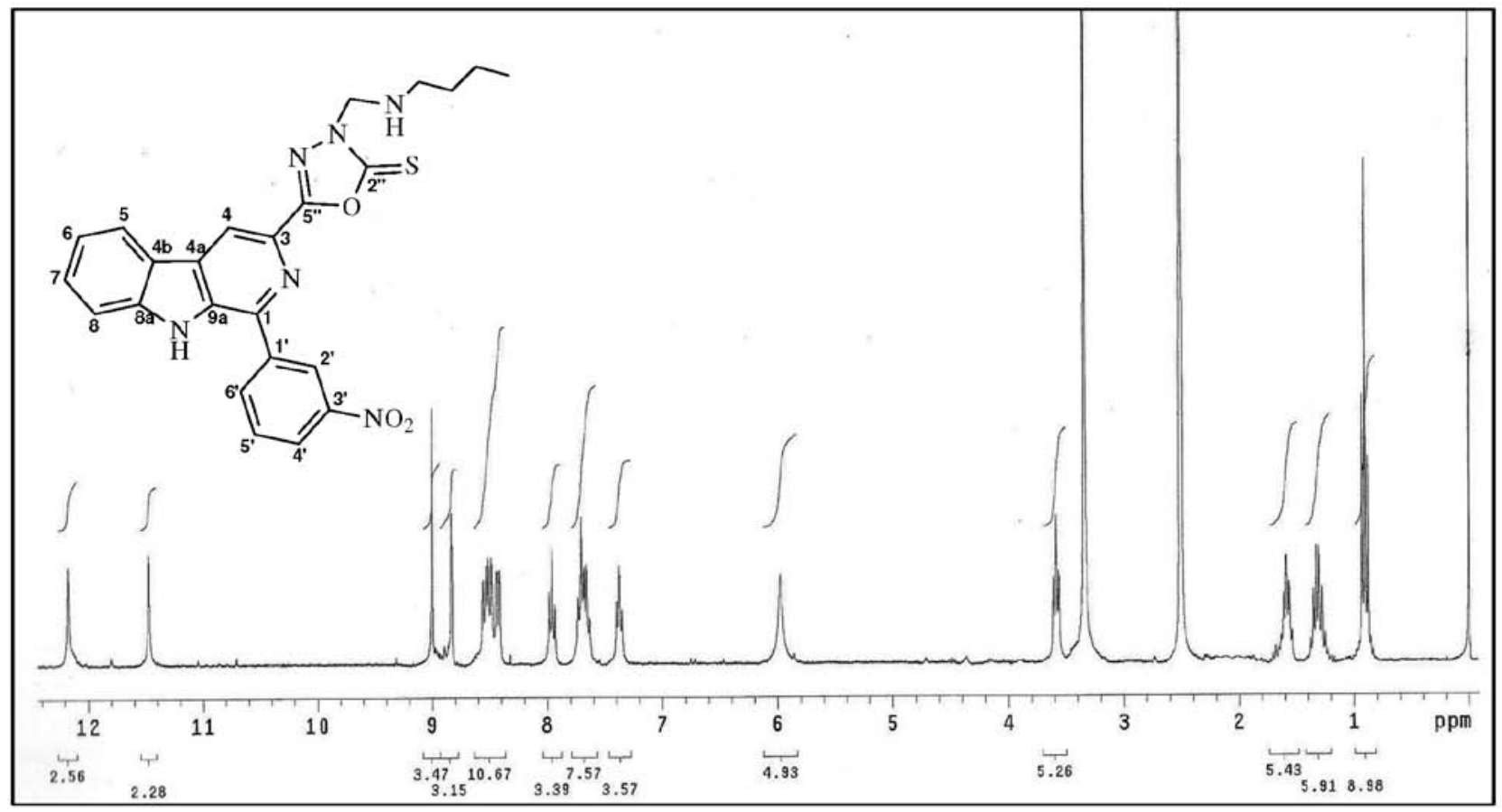

Figure S17. ${ }^{1} \mathrm{H}$ NMR spectra (300 MHz, DMSO- $d_{\sigma}$ ) of compound $\mathbf{3 b}$. 


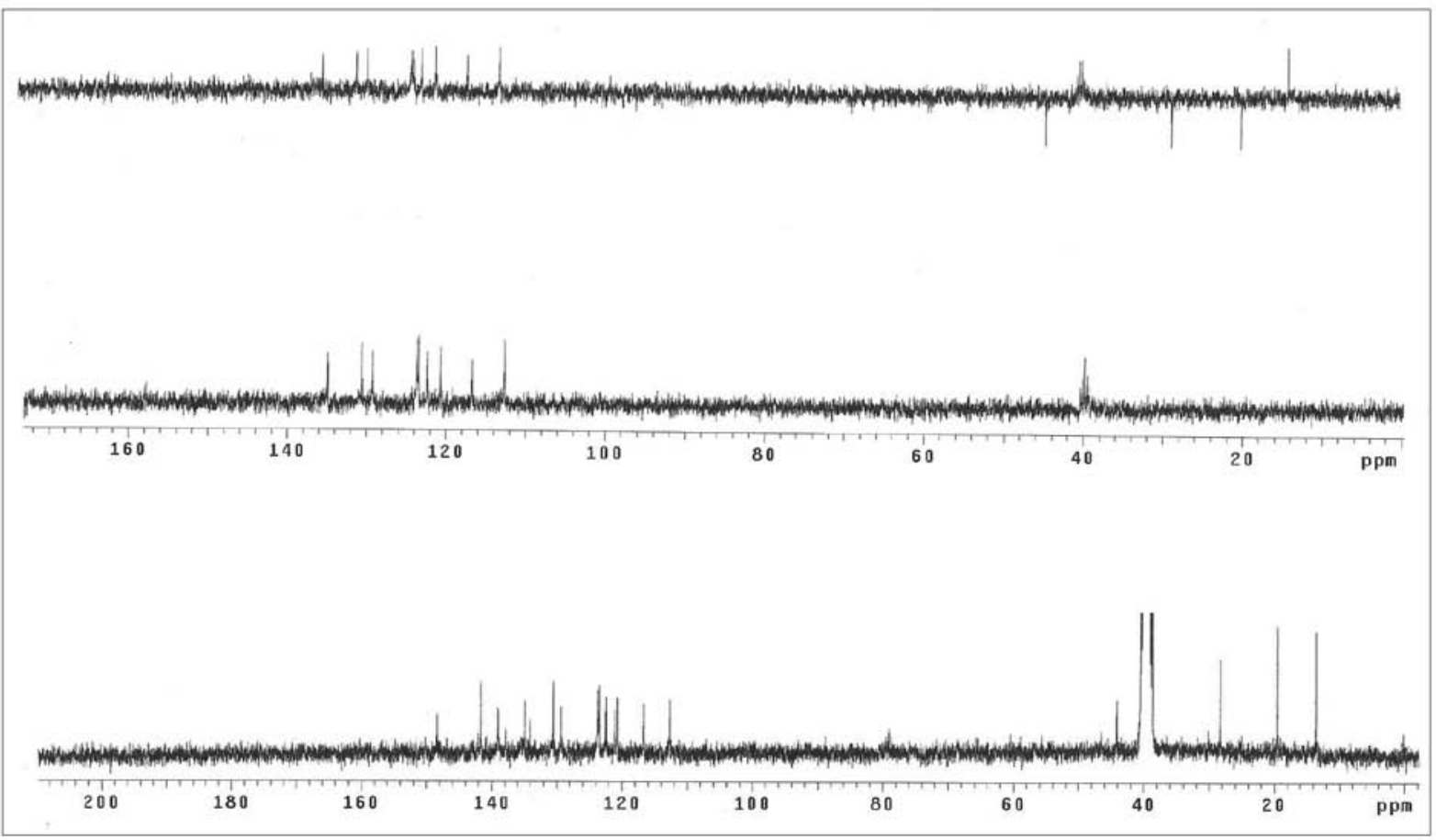

Figure S18. ${ }^{13} \mathrm{C}$ NMR / DEPT spectra (75.5 MHz, DMSO- $d_{6}$ ) of compound $3 \mathbf{b}$.

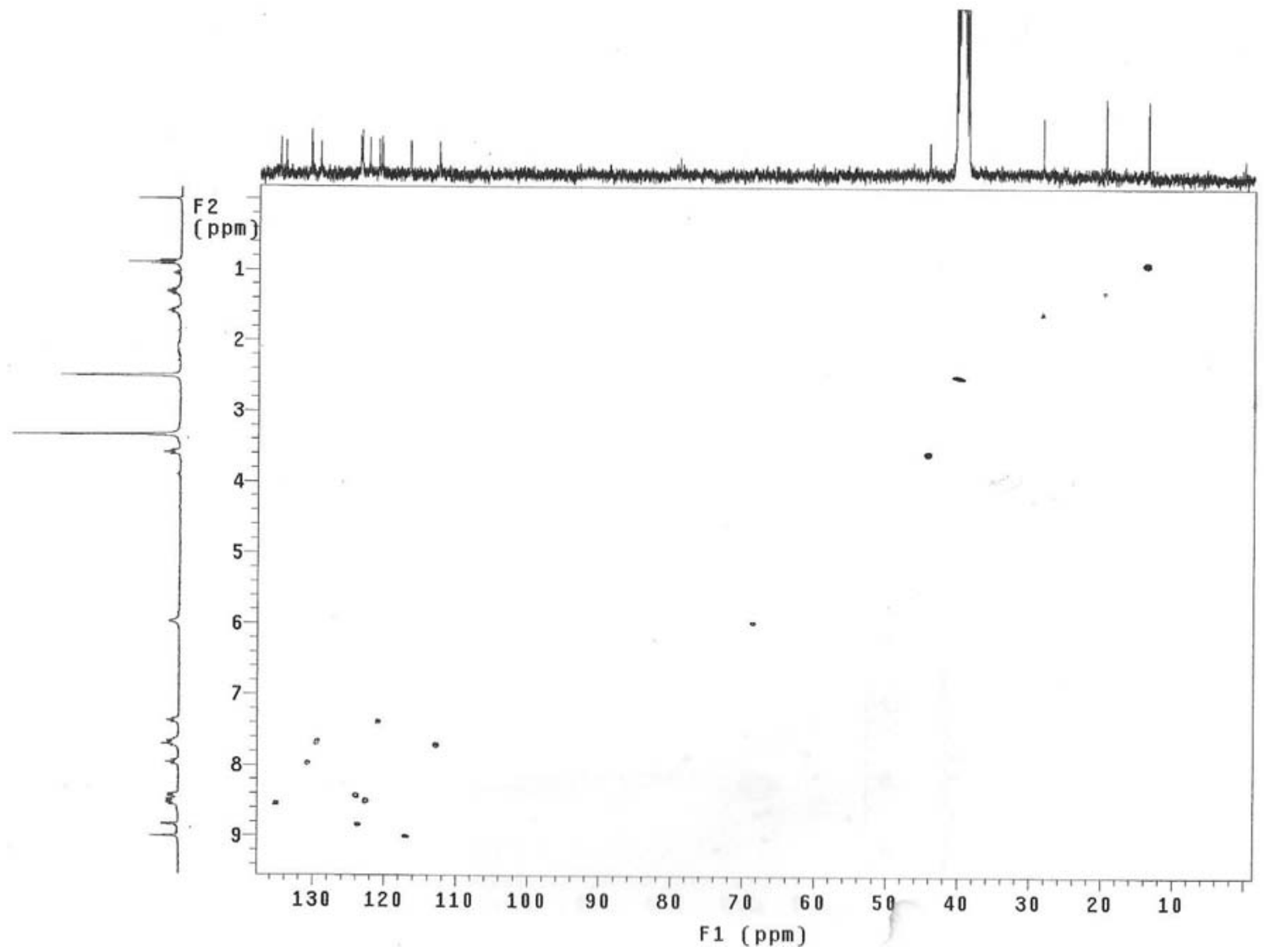

Figure S19. HMQC spectra of compound $\mathbf{3 b}$. 


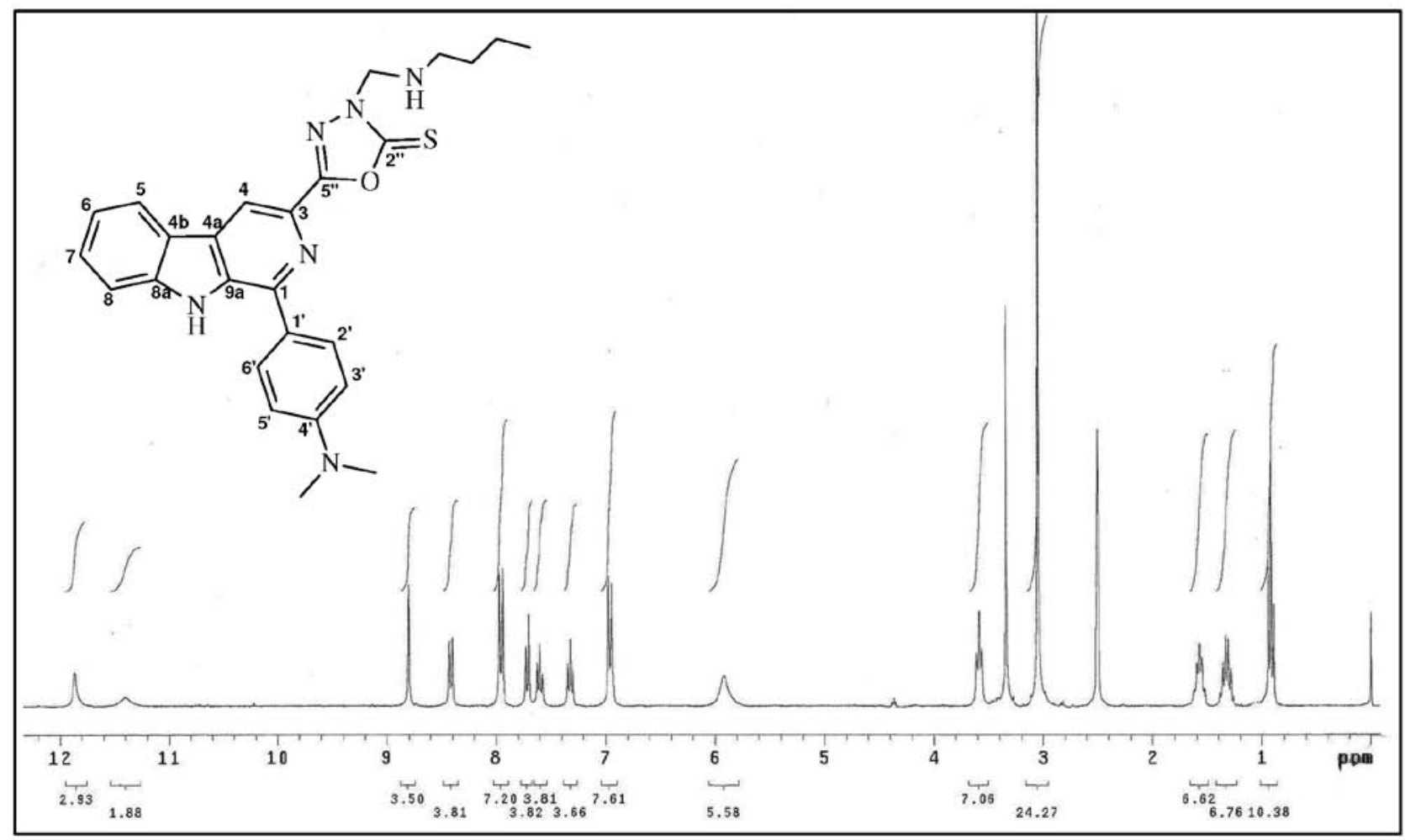

Figure S20. ${ }^{1} \mathrm{H}$ NMR spectra (300 MHz, DMSO- $d_{6}$ ) of compound 3c.

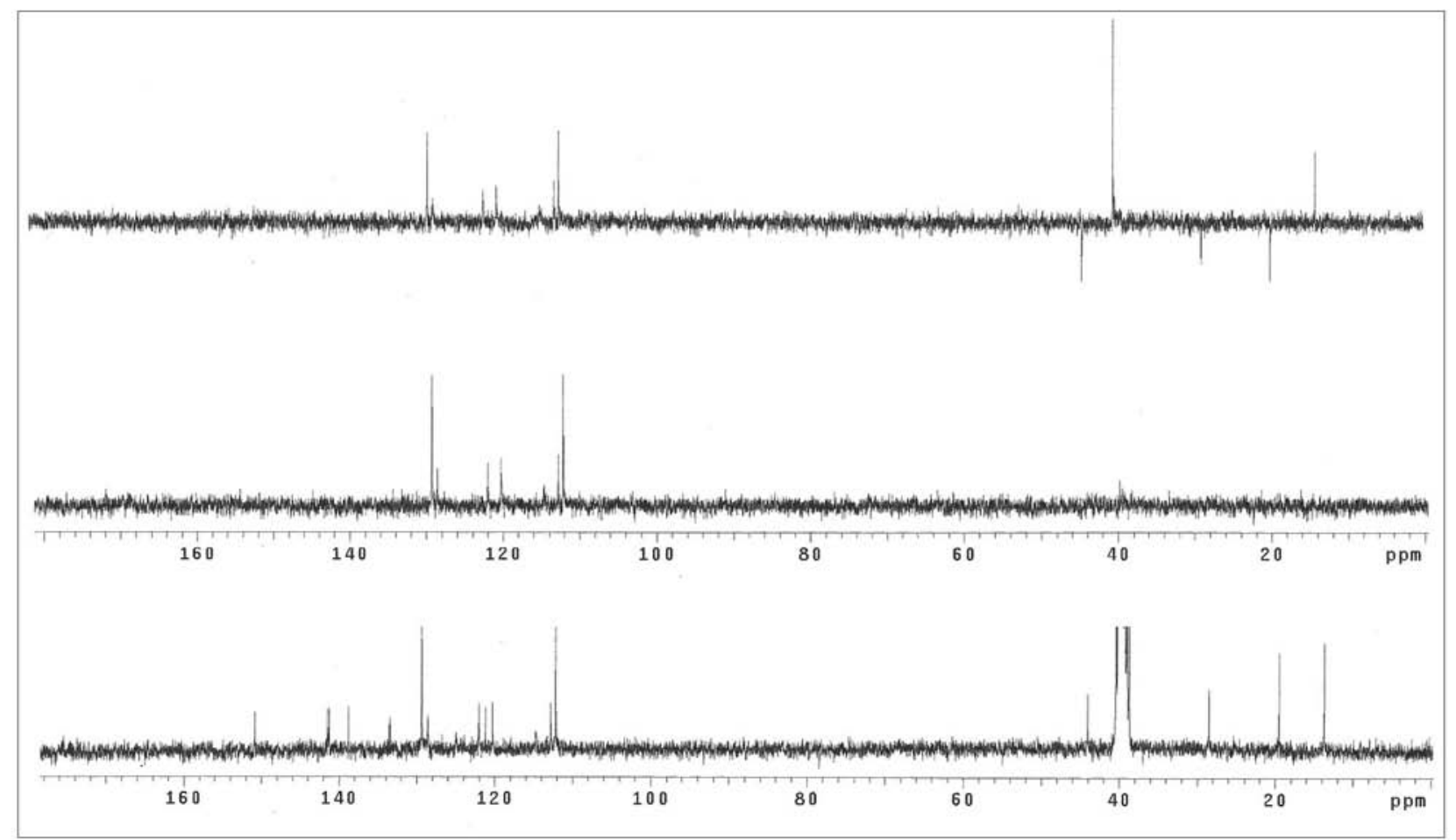

Figure S21. ${ }^{13} \mathrm{C}$ NMR / DEPT spectra (75.5 MHz, DMSO- $d_{6}$ ) of compound 3c. 


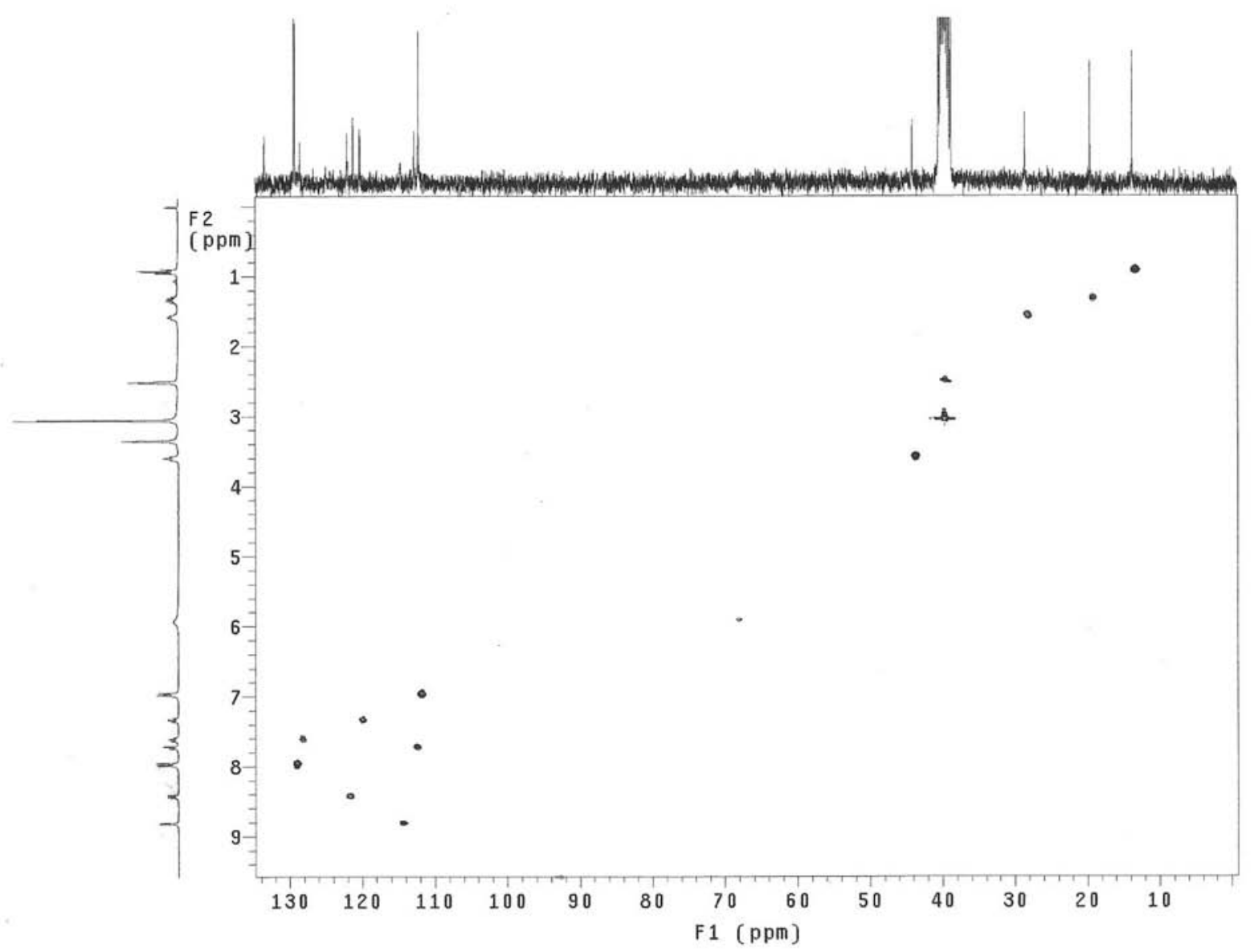

Figure S22. HMQC spectra of compound 3c.

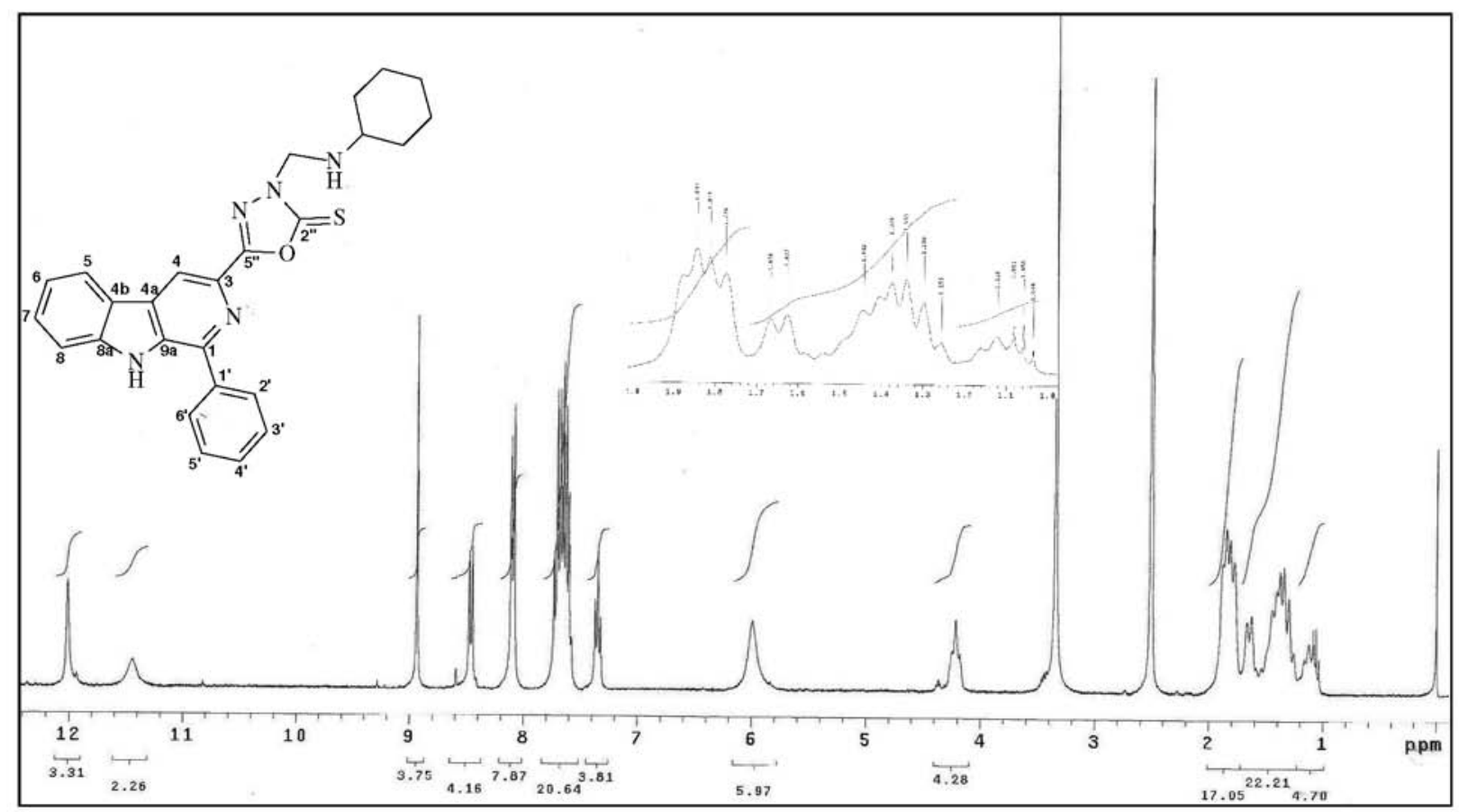

Figure S23. ${ }^{1} \mathrm{H}$ NMR spectra (300 MHz, DMSO- $\left.d_{6}\right)$ of compound 4a. 


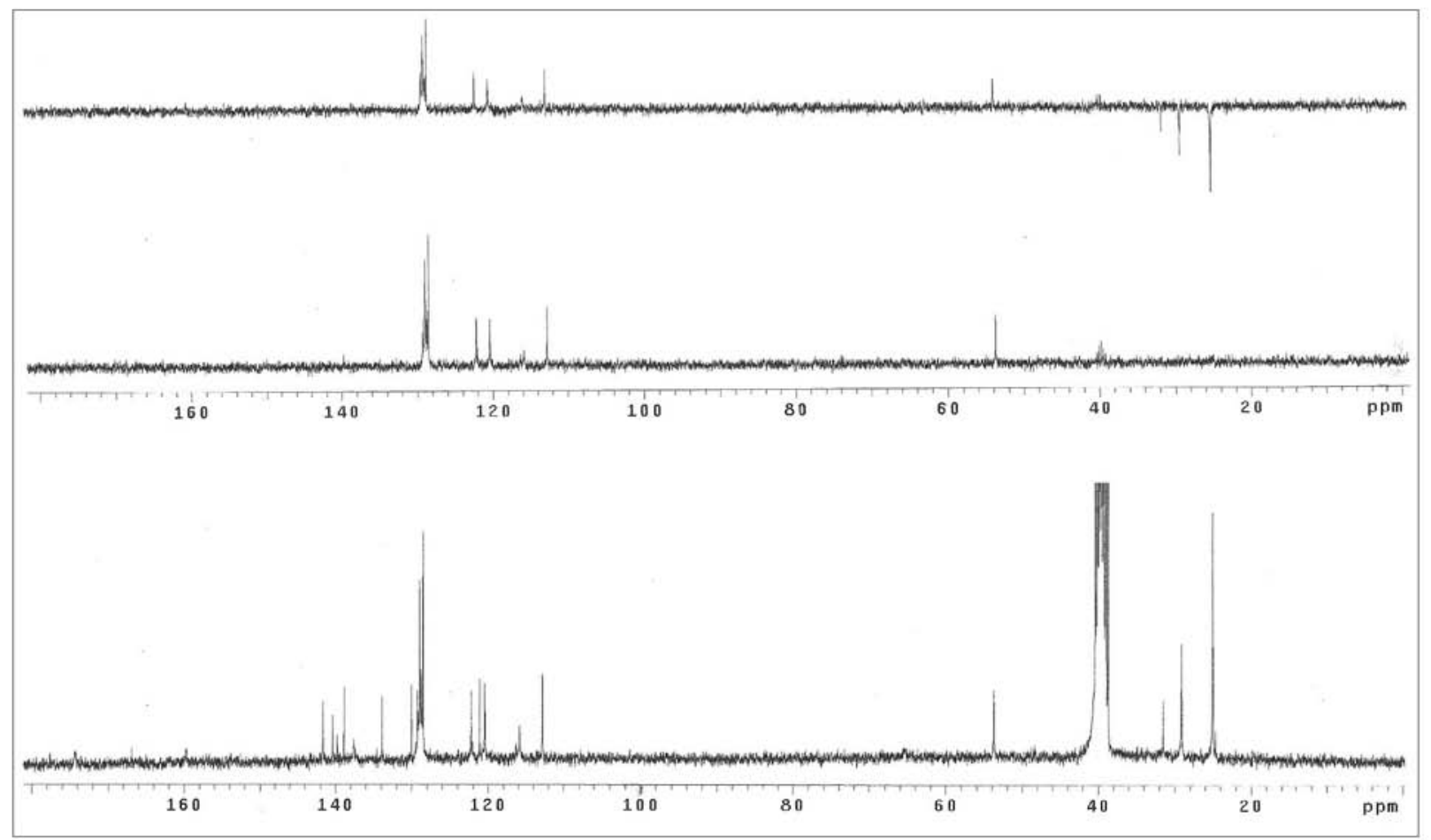

Figure S24. ${ }^{13} \mathrm{C}$ NMR / DEPT spectra (75.5 MHz, DMSO- $d_{\sigma}$ ) of compound 4 a.

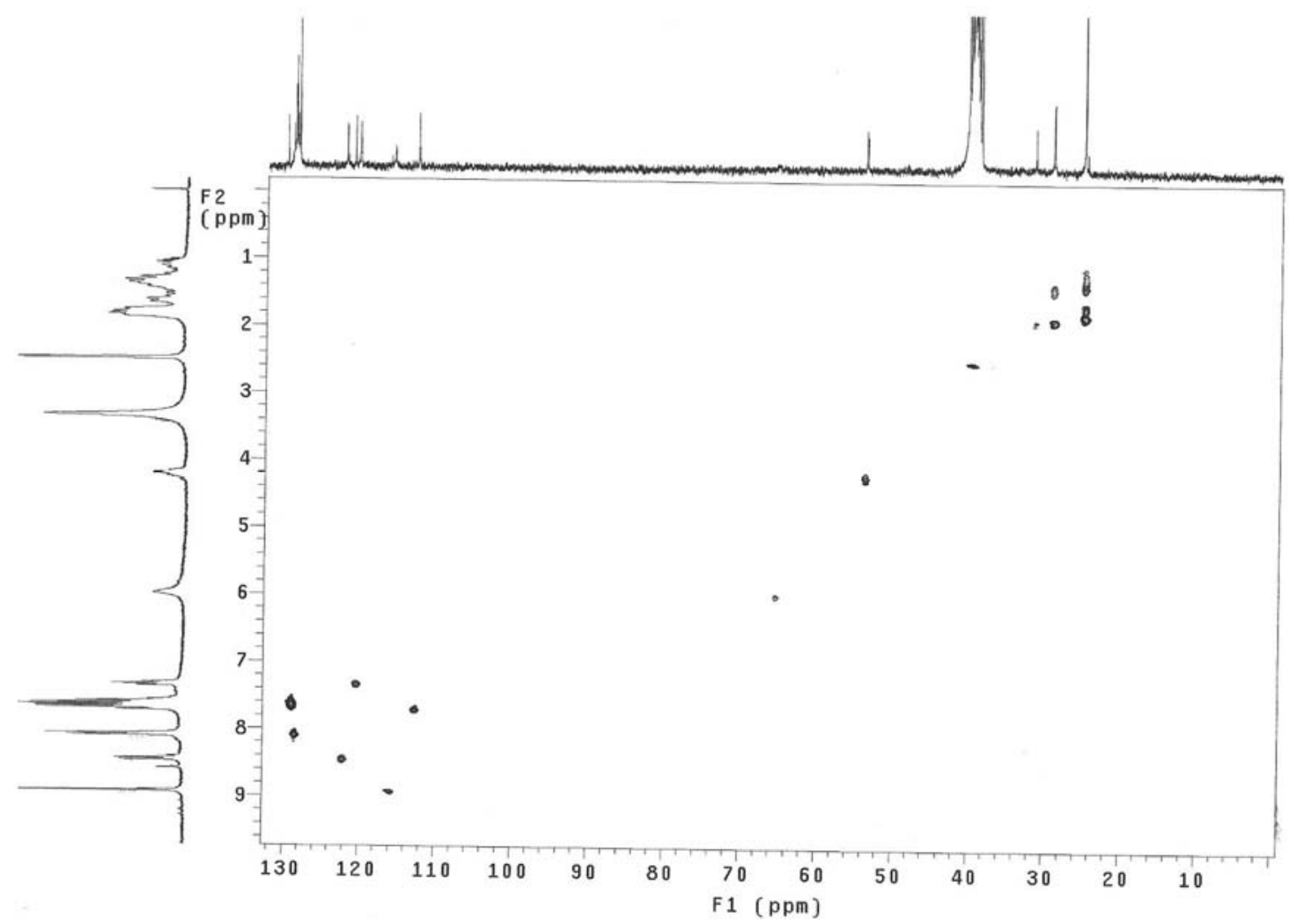

Figure S25. HMQC spectra of compound 4a. 


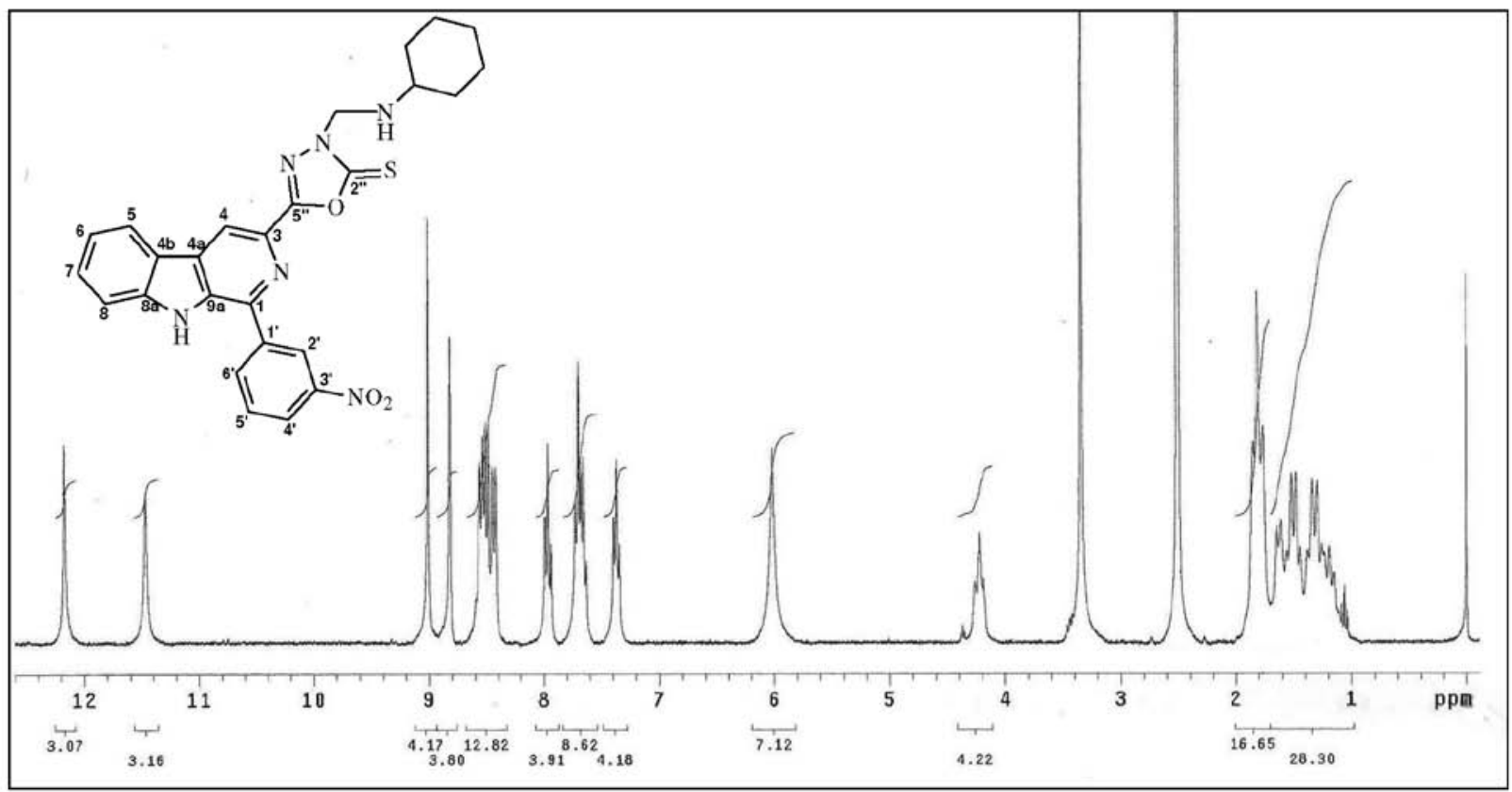

Figure S26. ${ }^{1} \mathrm{H}$ NMR spectra (300 MHz, DMSO- $d_{\sigma}$ ) of compound $\mathbf{4 b}$.

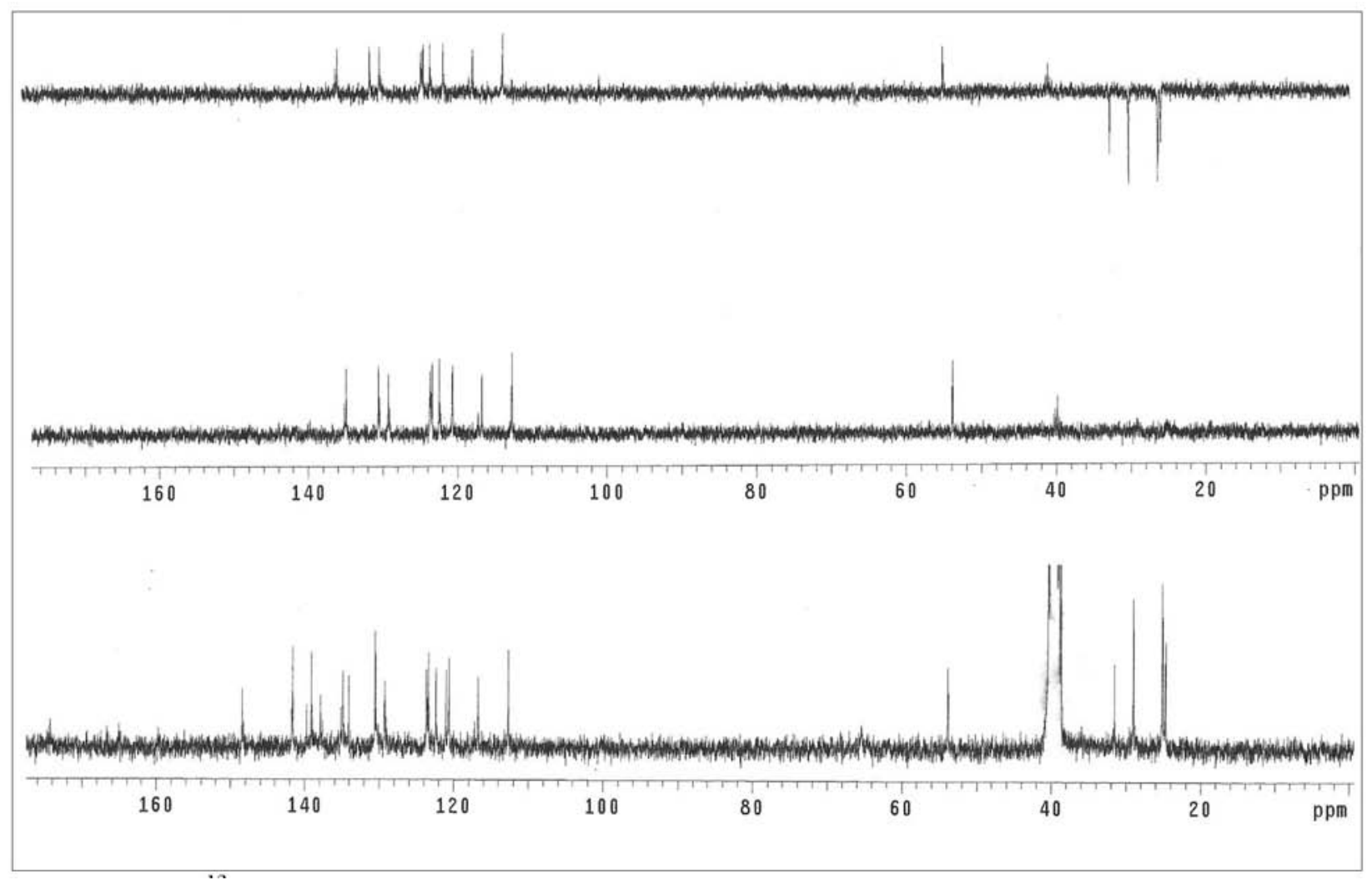

Figure S27. ${ }^{13} \mathrm{C}$ NMR / DEPT spectra (75.5 MHz, DMSO- $d_{6}$ ) of compound $\mathbf{4 b}$. 


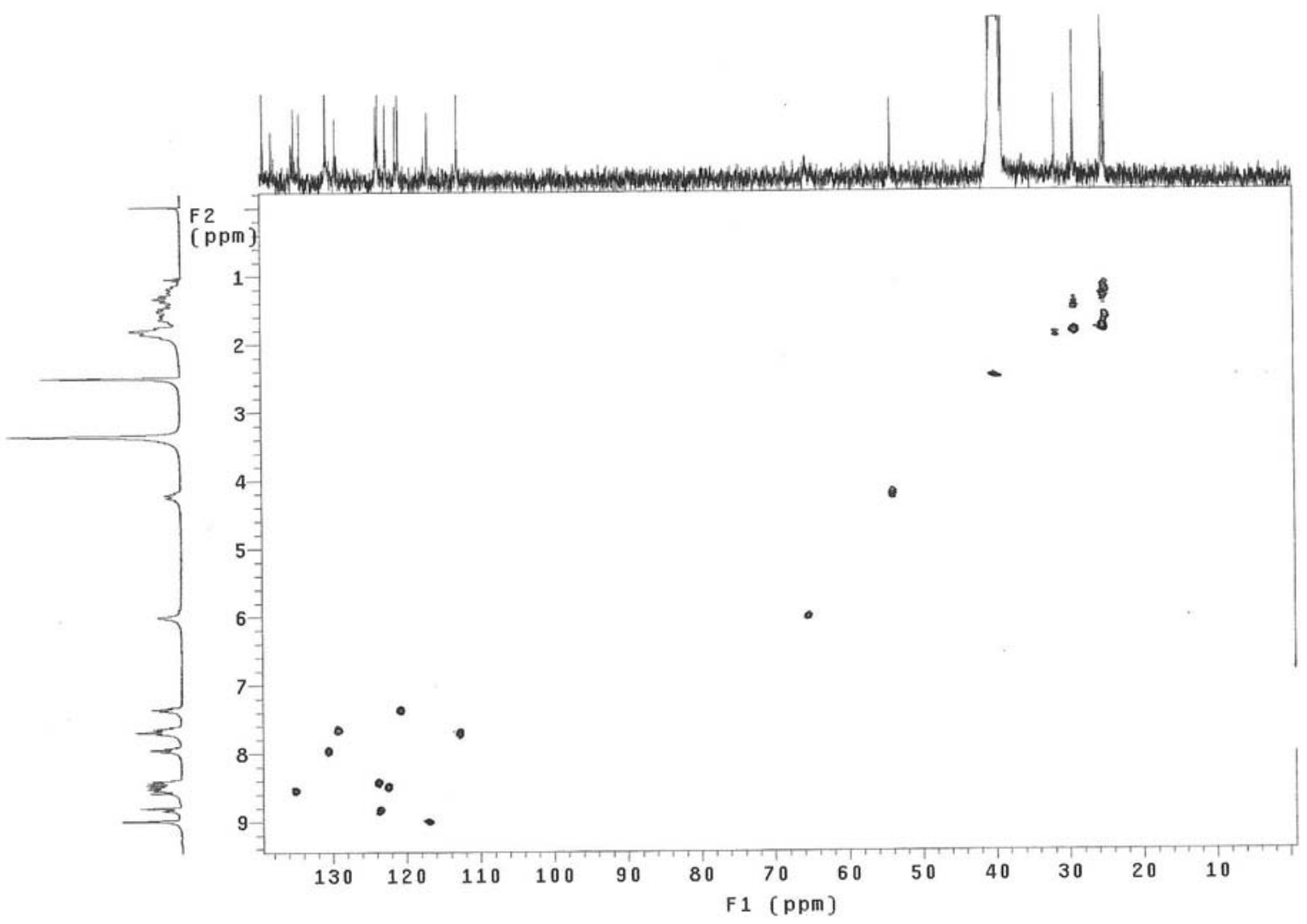

Figure S28. HMQC spectra of compound $\mathbf{4 b .}$

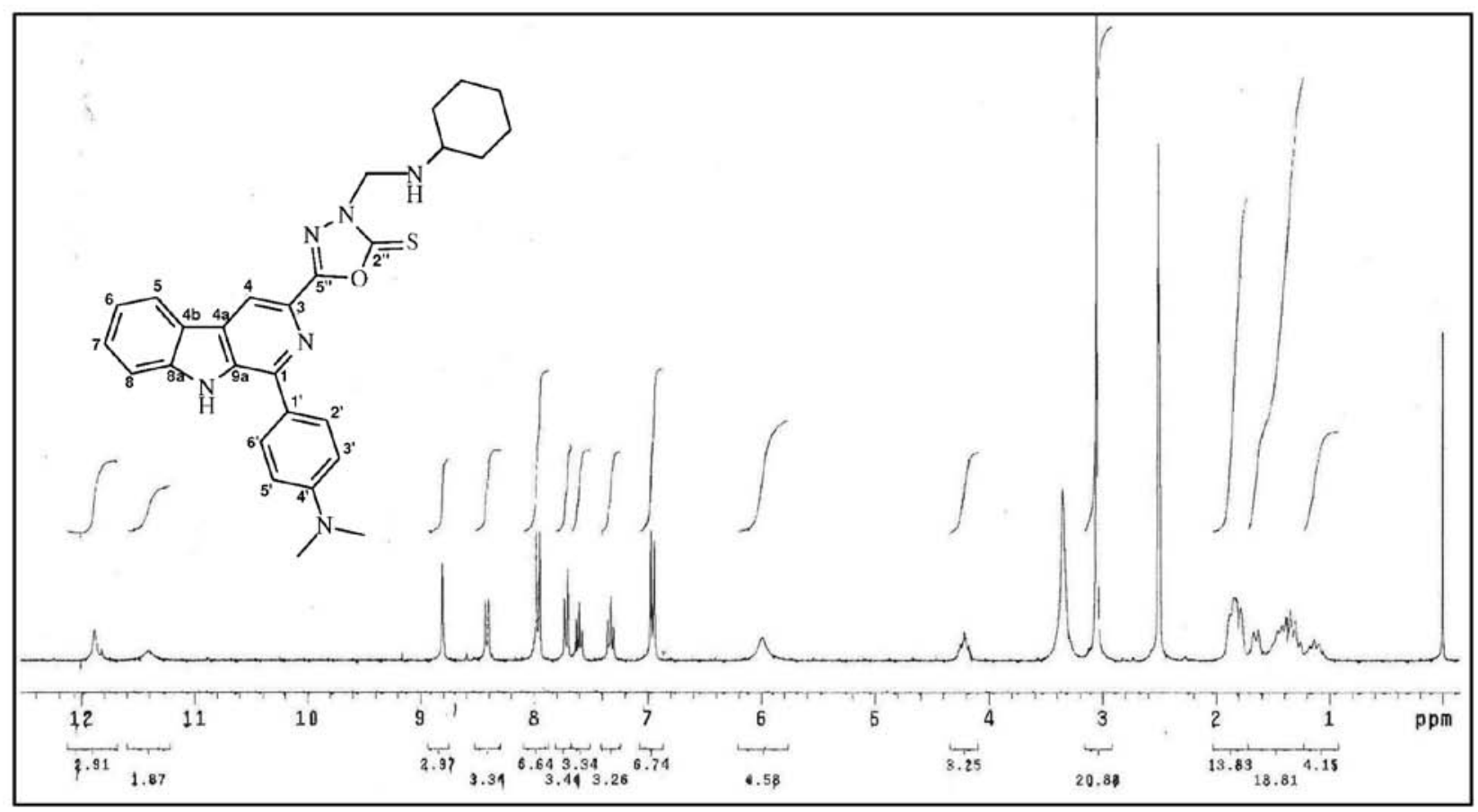

Figure S29. ${ }^{1} \mathrm{H}$ NMR spectra (300 MHz, DMSO- $d_{6}$ ) of compound $4 \mathbf{4}$. 


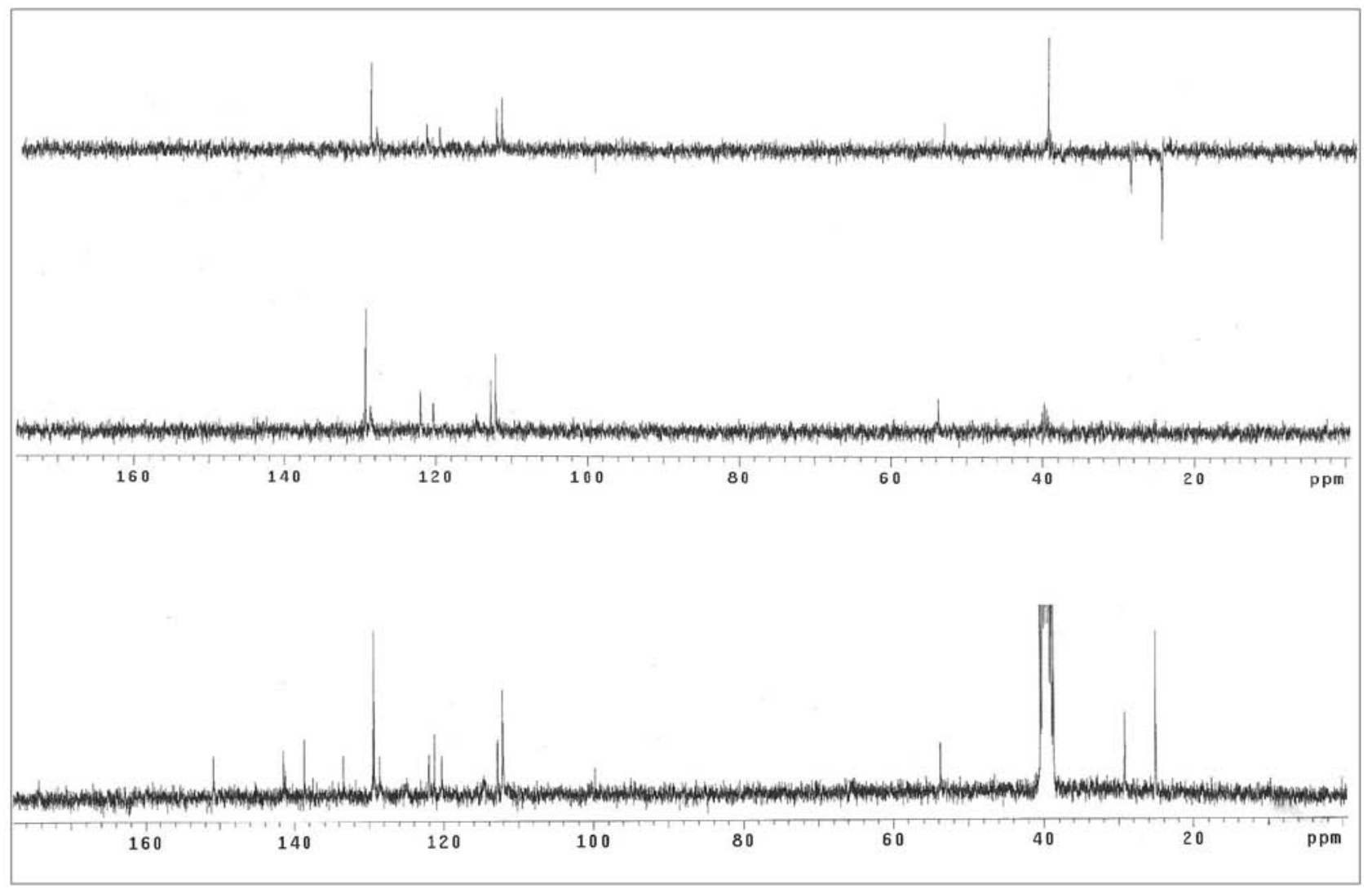

Figure S30. ${ }^{13} \mathrm{C}$ NMR / DEPT spectra (75.5 MHz, DMSO- $d_{6}$ ) of compound 4c.

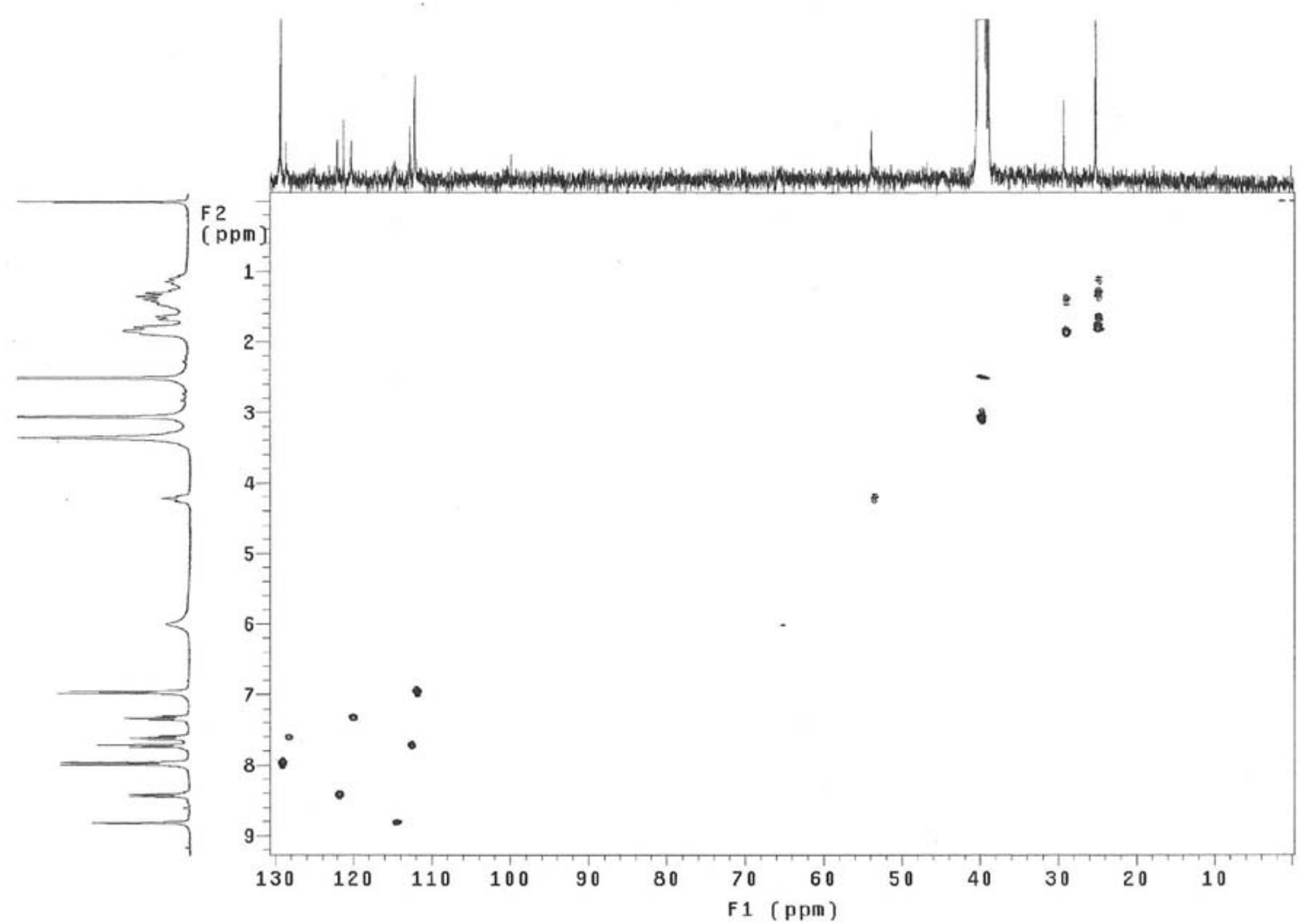

Figure S31. HMQC spectra of compound 4c. 


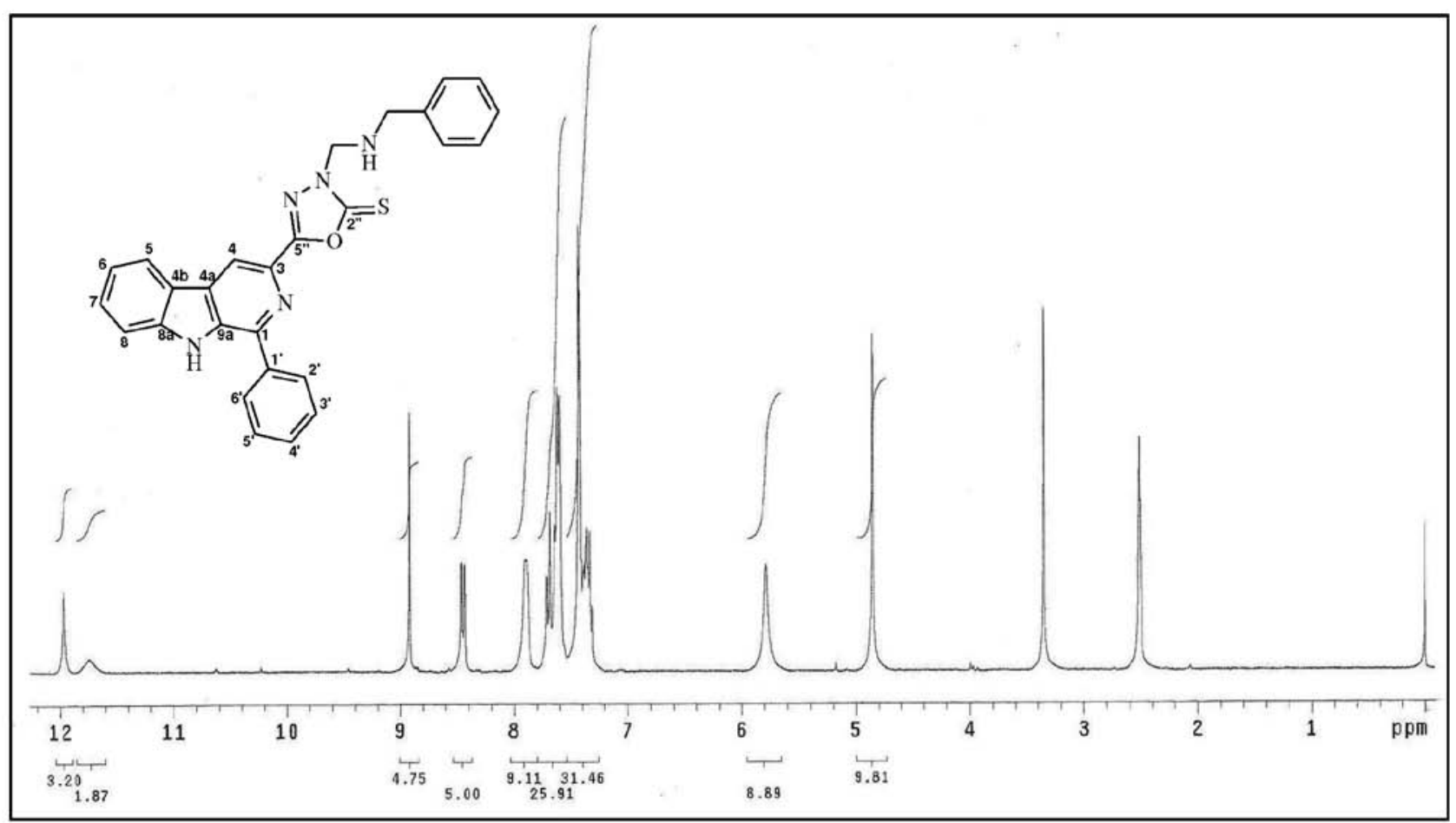

Figure S32. ${ }^{1} \mathrm{H}$ NMR spectra (300 MHz, DMSO- $d_{6}$ ) of compound $\mathbf{5 a}$.

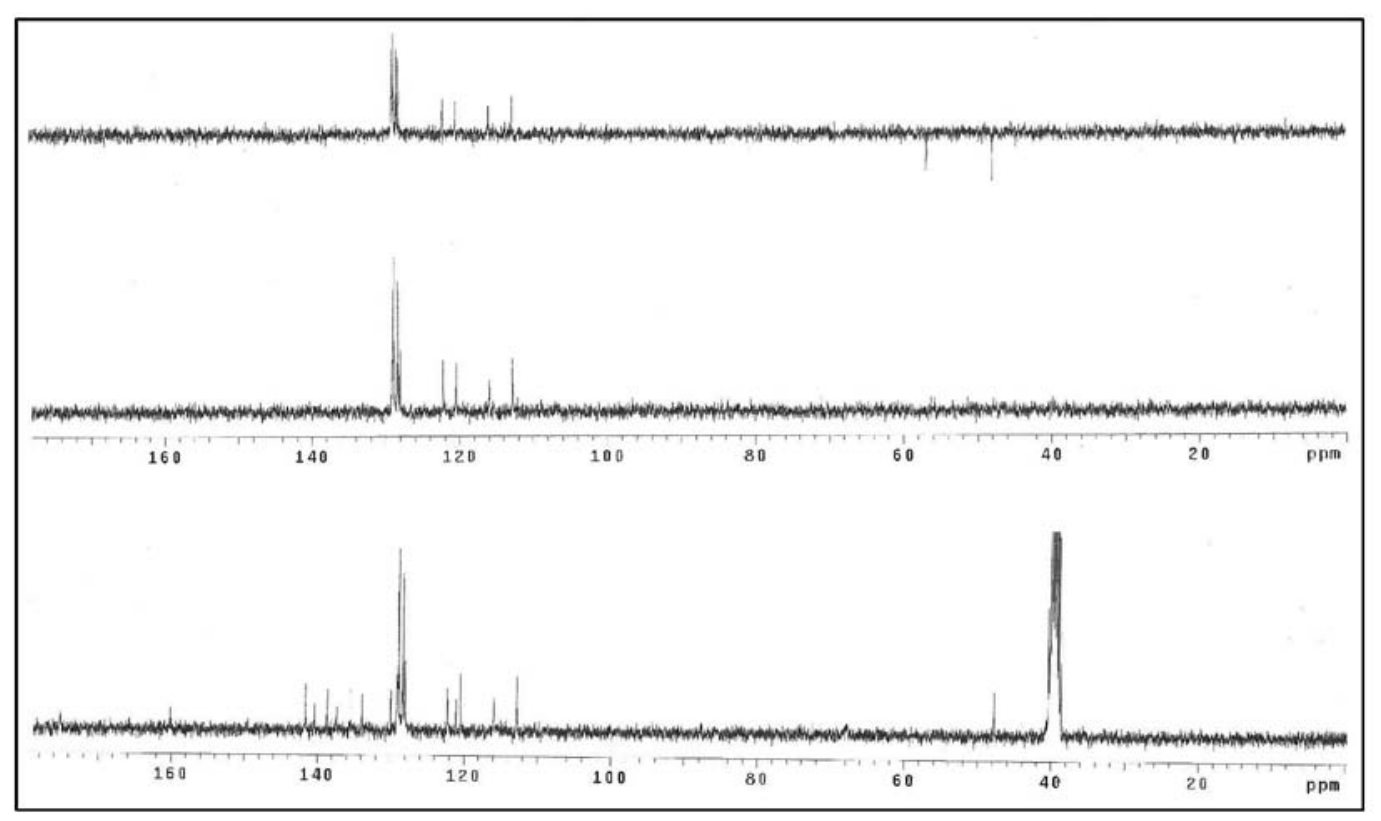

Figure S33. ${ }^{13} \mathrm{C}$ NMR / DEPT spectra (75.5 MHz, DMSO- $d_{6}$ ) of compound 5a. 


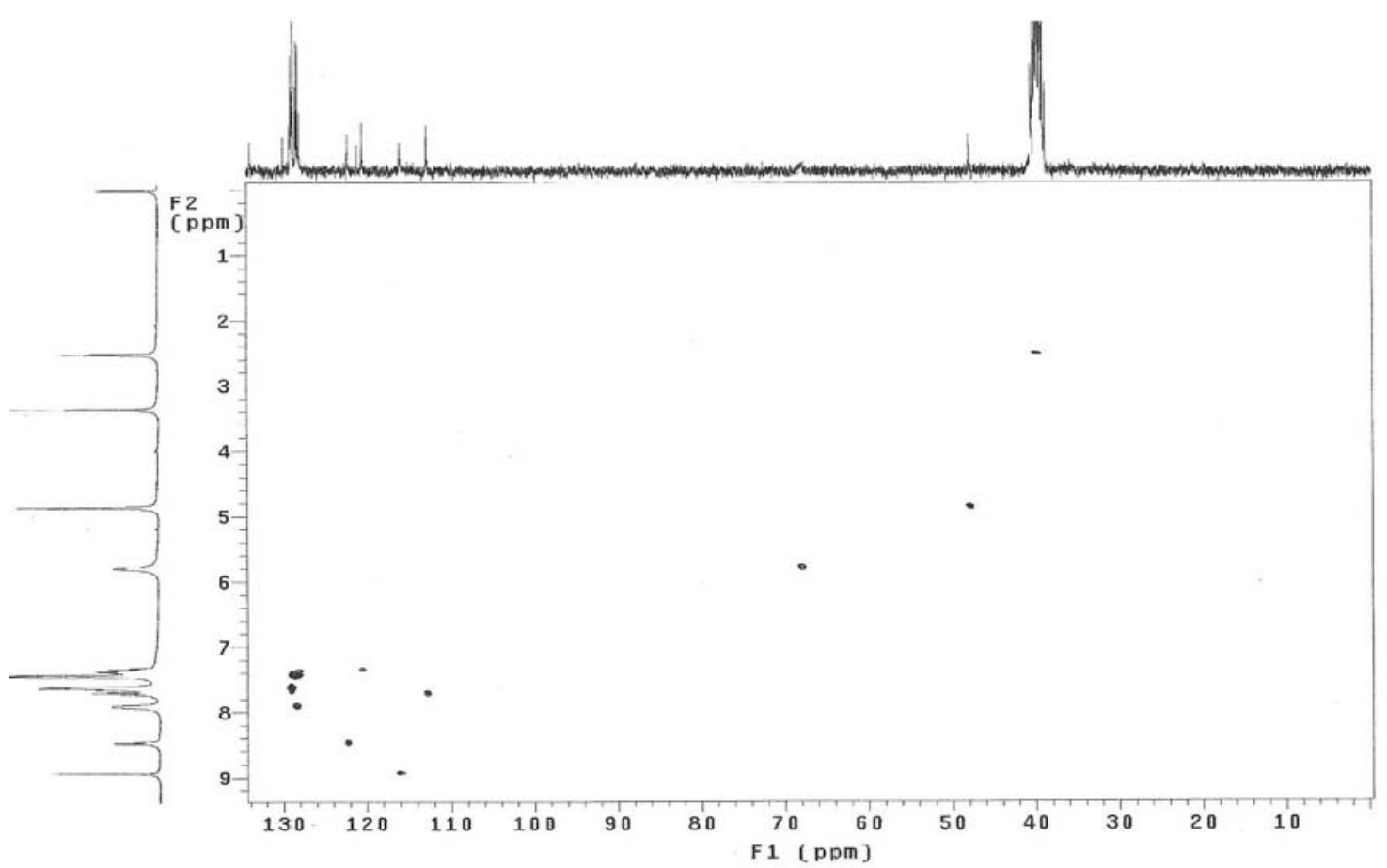

Figure S34. HMQC spectra of compound 5a.

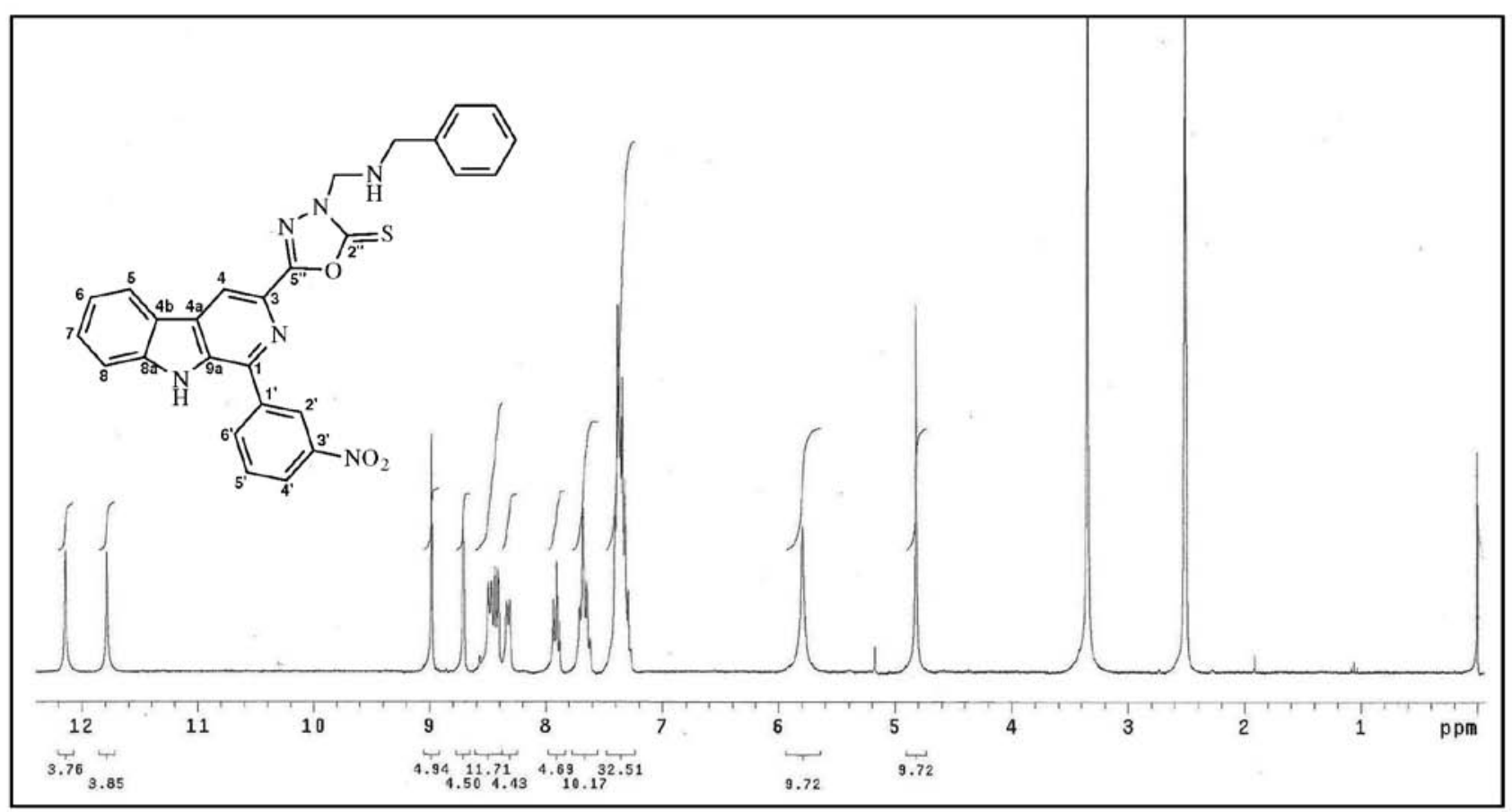

Figure S35. ${ }^{1} \mathrm{H}$ NMR spectra (300 MHz, DMSO- $d_{6}$ ) of compound $\mathbf{5 b}$. 


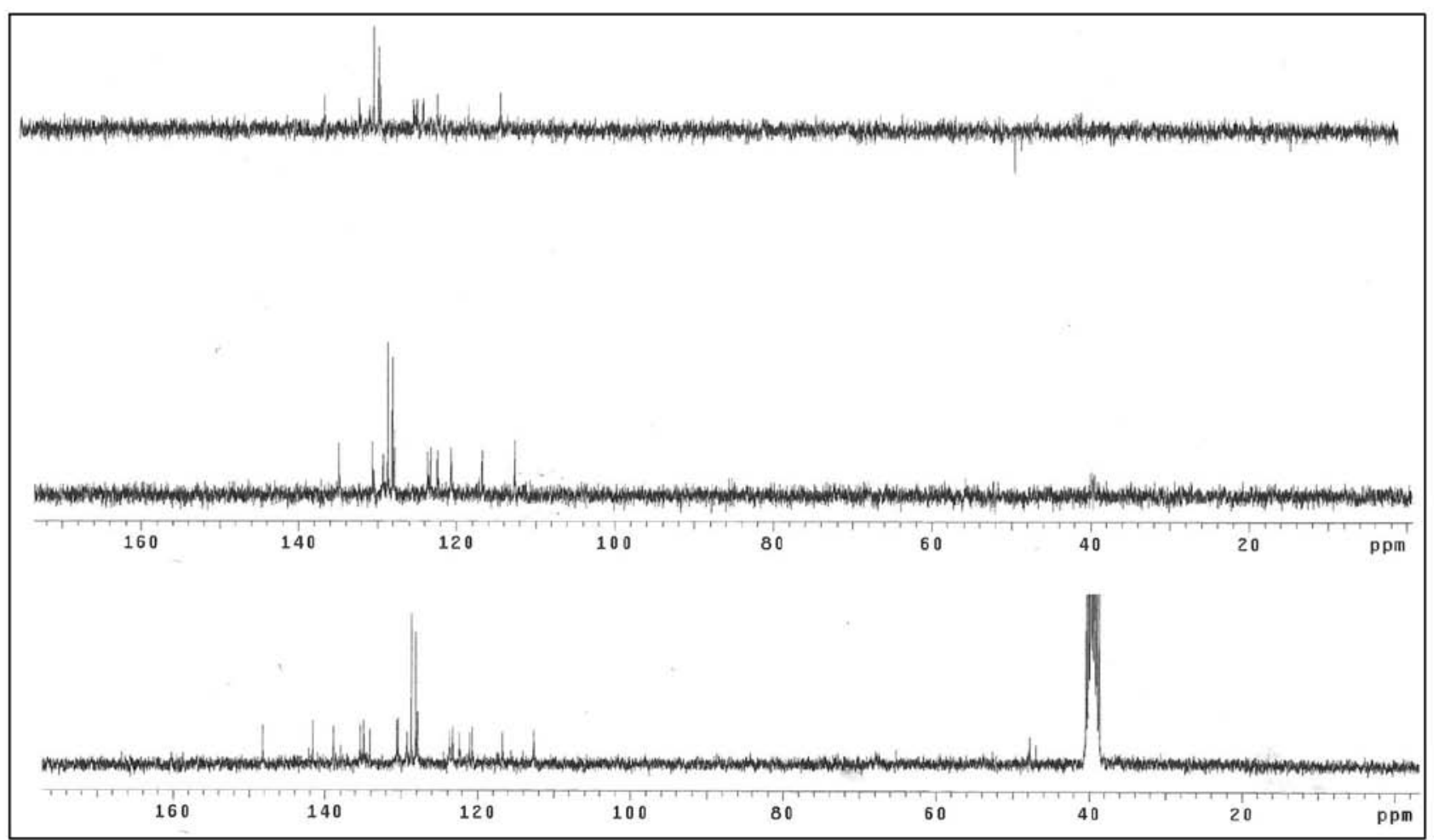

Figure S36. ${ }^{13} \mathrm{C}$ NMR / DEPT spectra (75.5 MHz, DMSO- $d_{6}$ ) of compound $\mathbf{5 b}$.

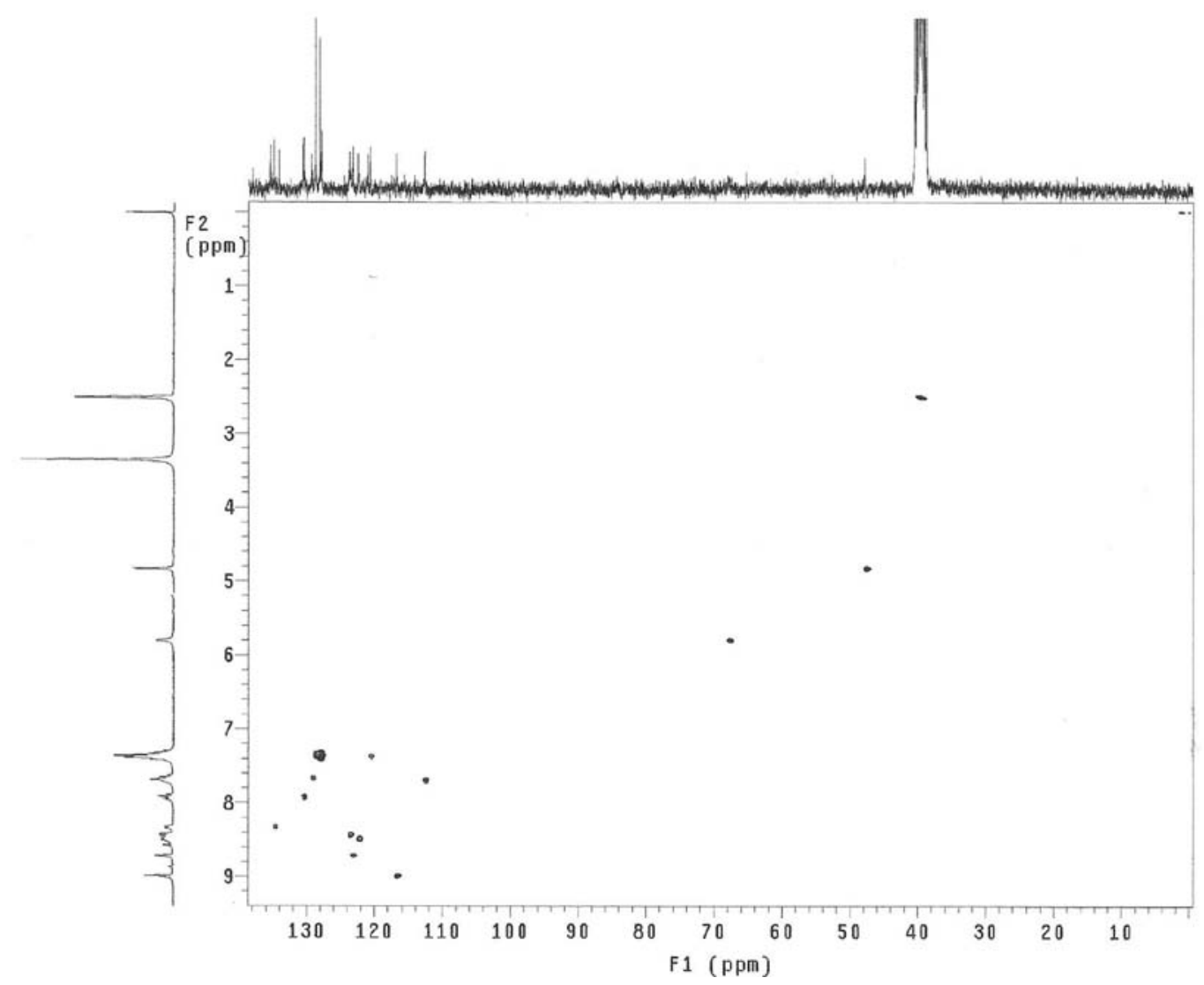

Figure S37. HMQC spectra of compound $\mathbf{5 b}$. 


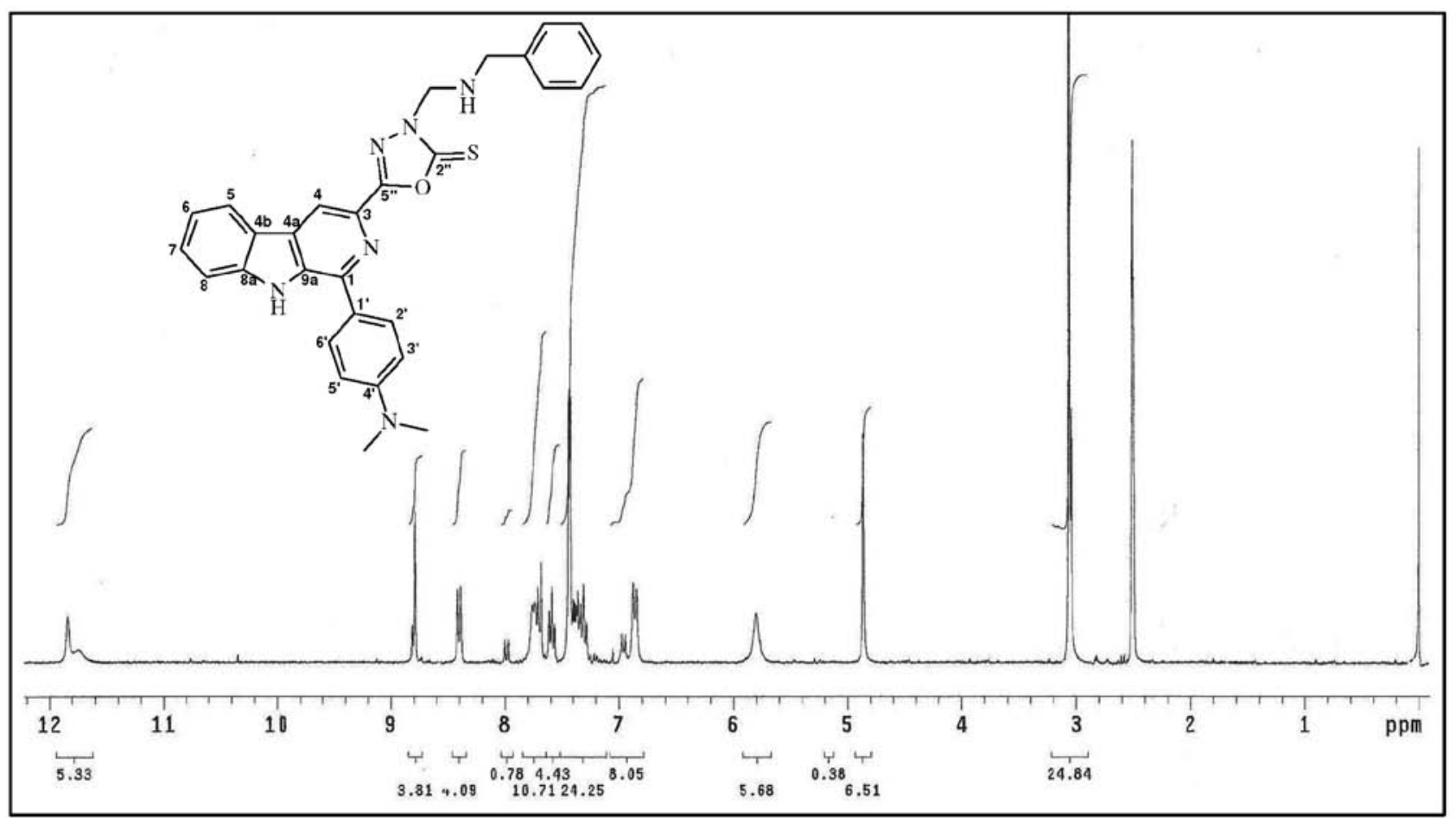

Figure S38. ${ }^{1} \mathrm{H}$ NMR spectra ( $\left.300 \mathrm{MHz}, \mathrm{DMSO}-d_{6}\right)$ of compound $\mathbf{5 c}$.

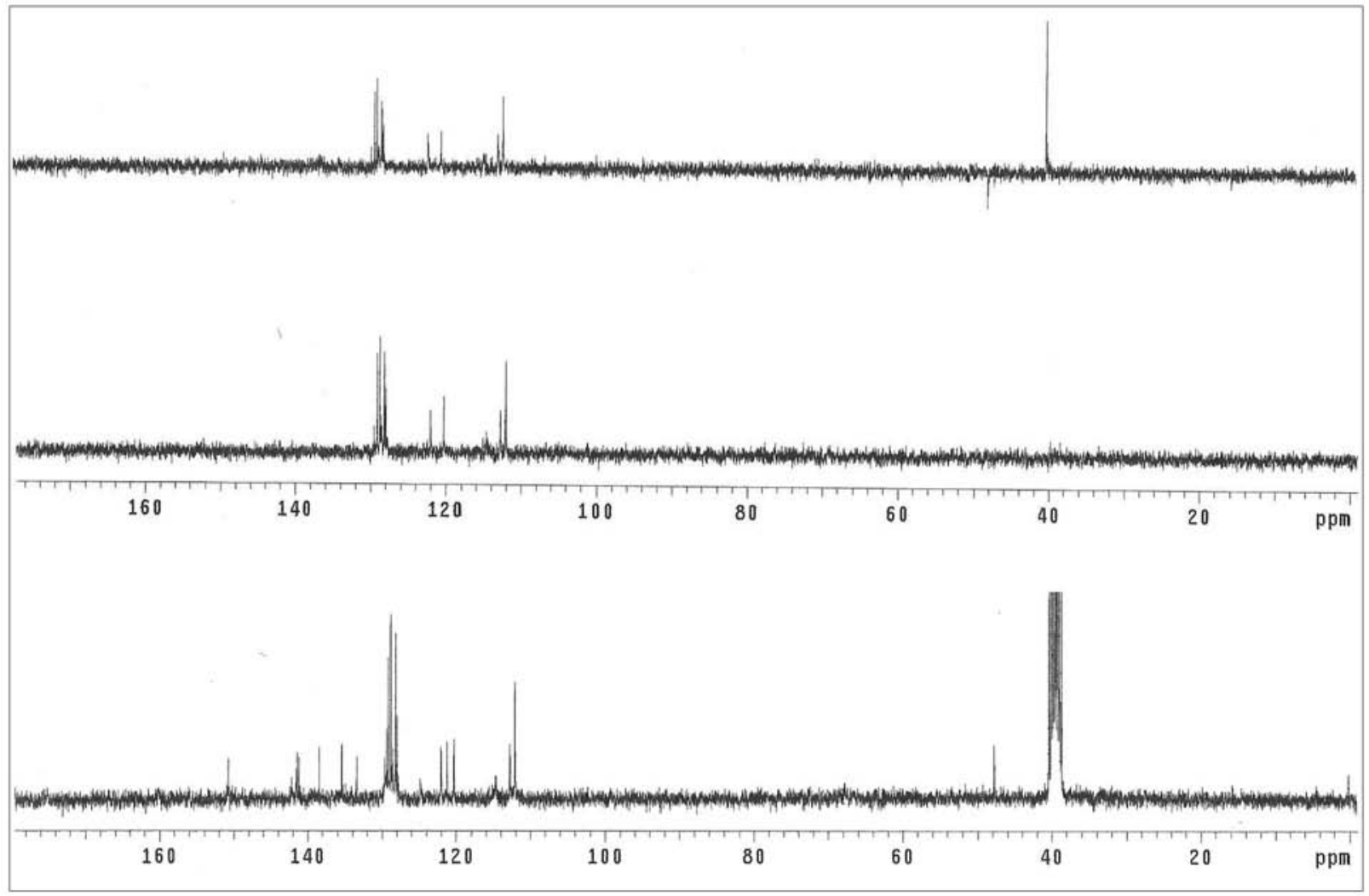

Figure S39. ${ }^{13} \mathrm{C}$ NMR / DEPT spectra (75.5 MHz, DMSO- $d_{6}$ ) of compound 5c. 


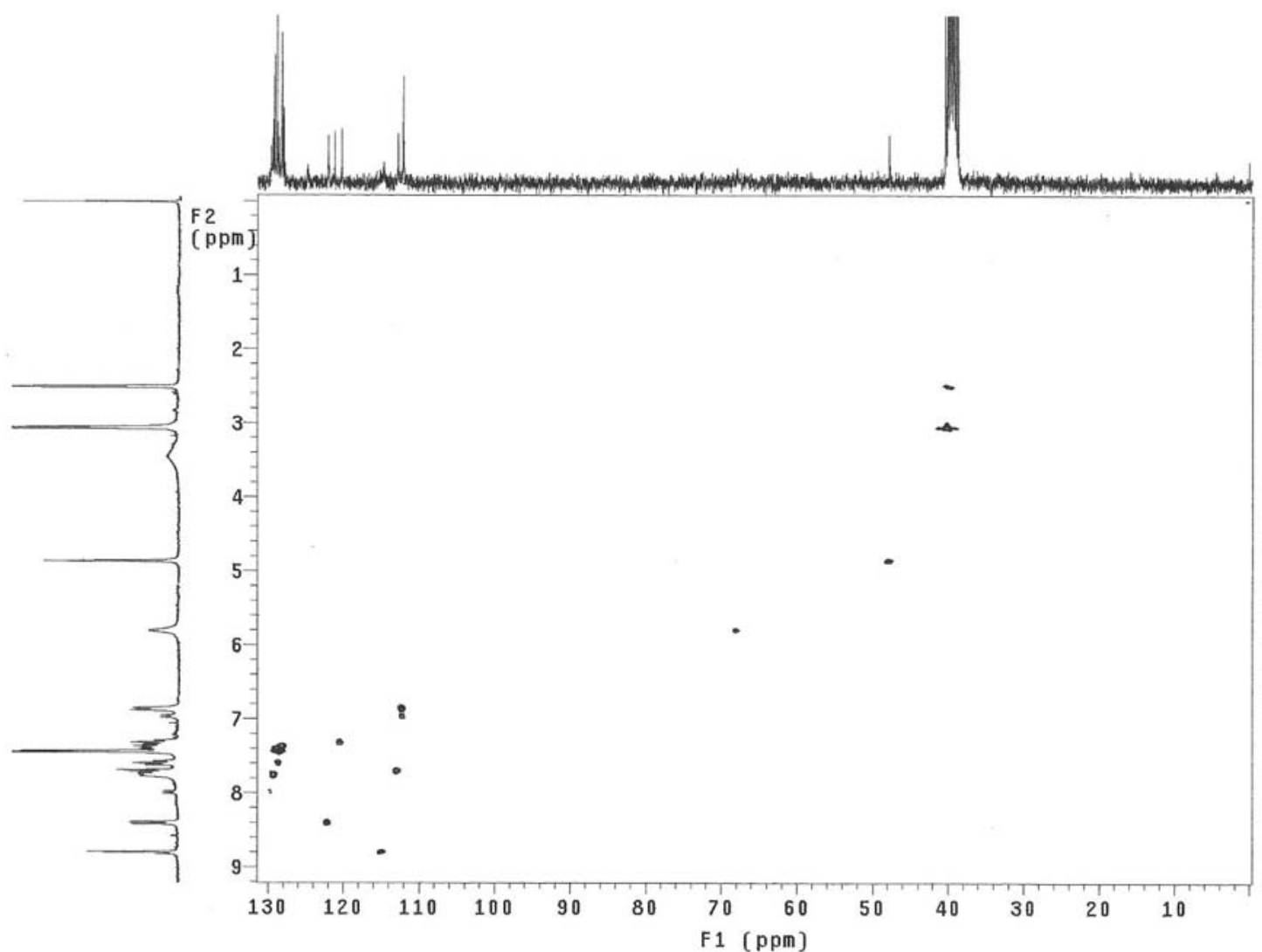

Figure S40. HMQC spectra of compound 5c.

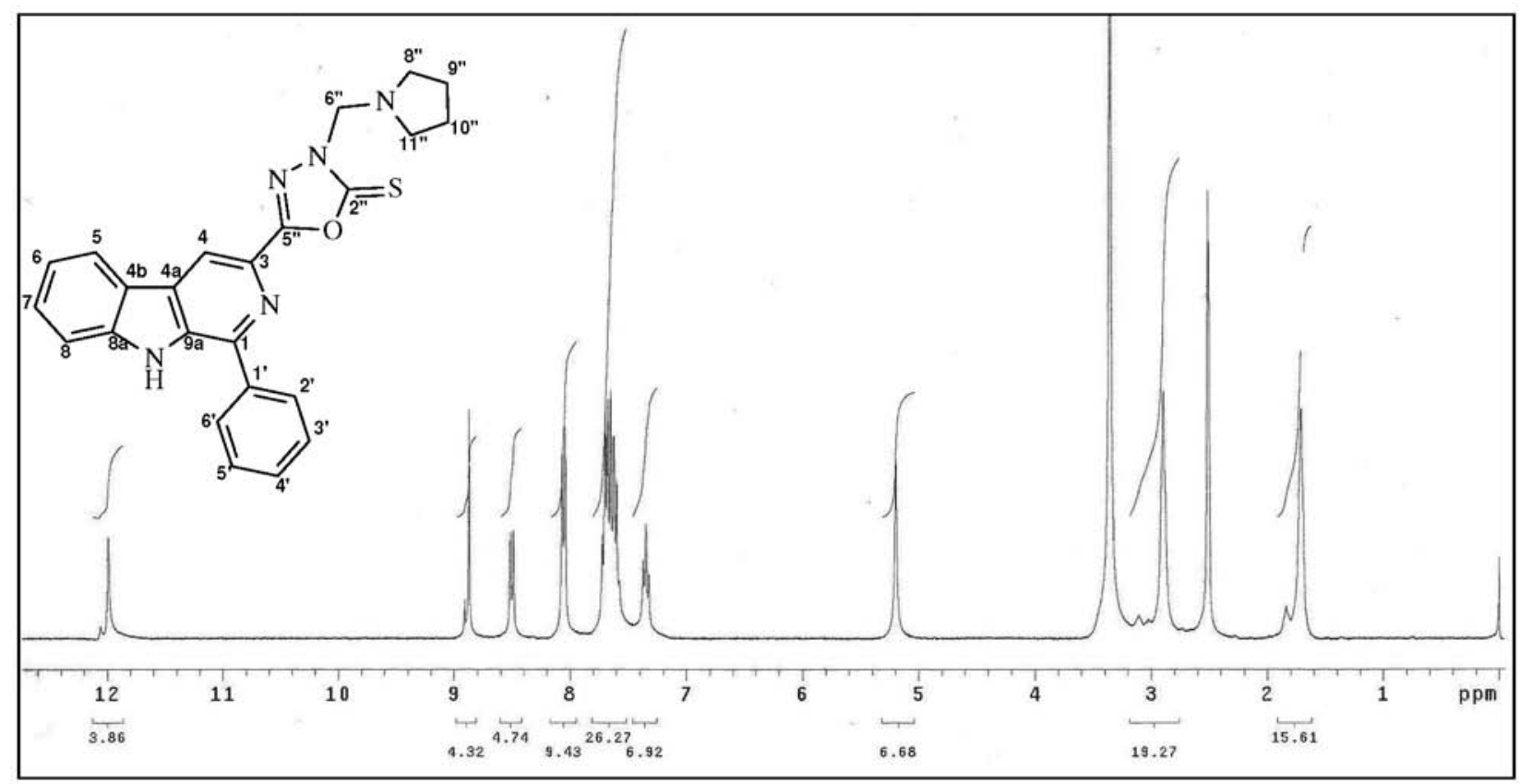

Figure S41. ${ }^{1} \mathrm{H}$ NMR spectra (300 MHz, DMSO- $d_{6}$ ) of compound 6 a. 


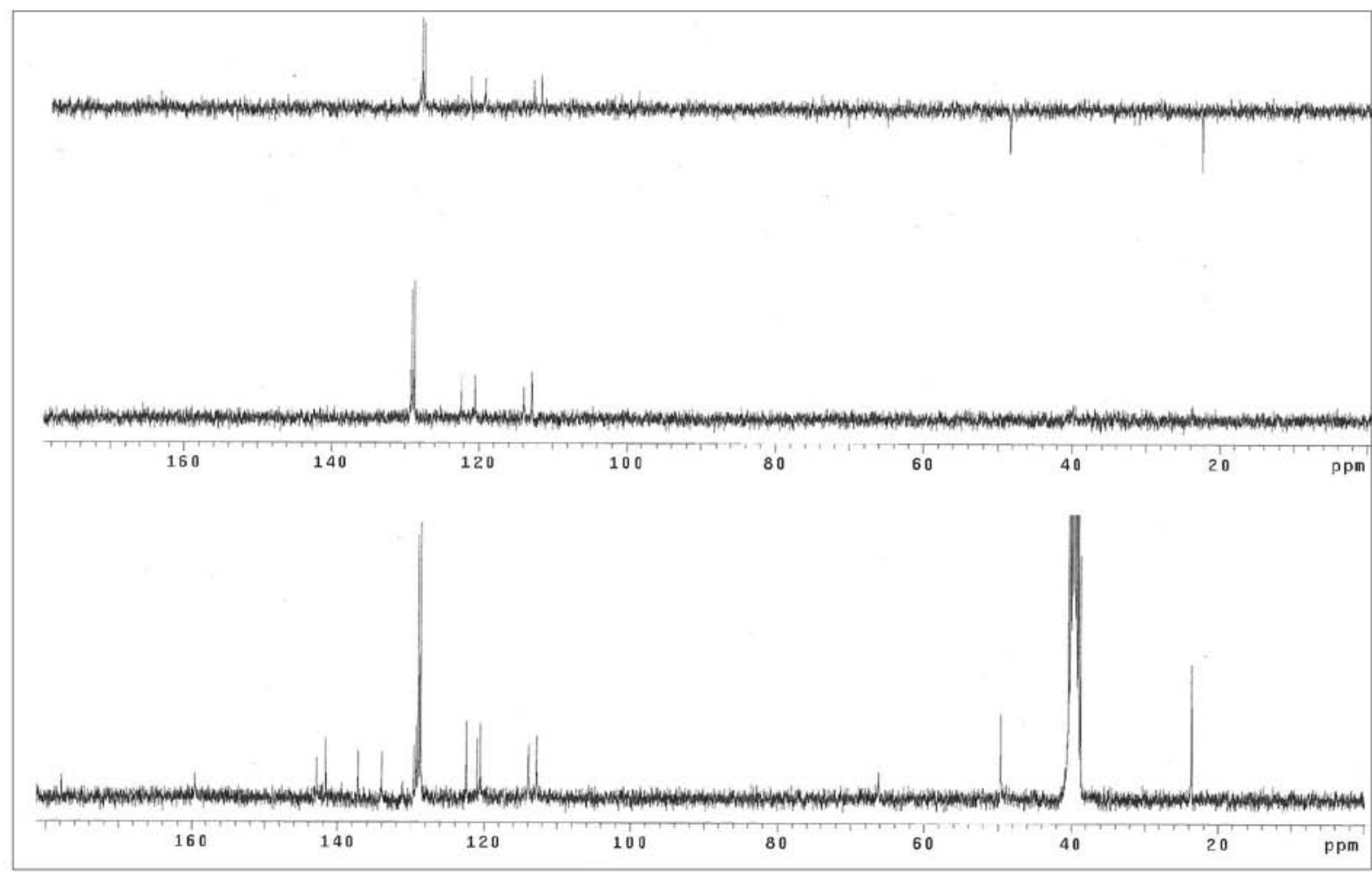

Figure S42. ${ }^{13} \mathrm{C}$ NMR / DEPT spectra (75.5 MHz, DMSO- $\left.d_{6}\right)$ of compound $6 \mathbf{a}$.

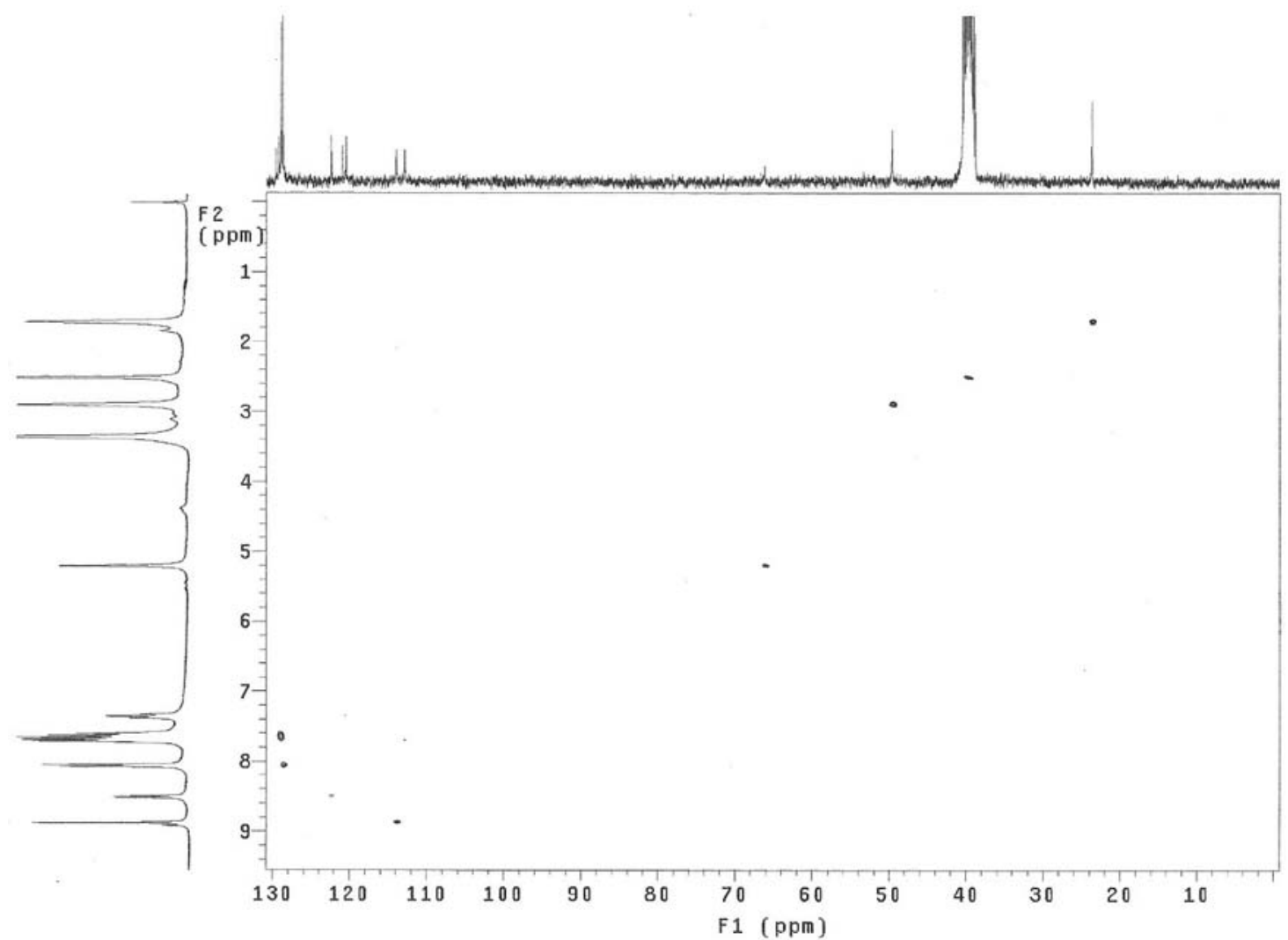

Figure S43. HMQC spectra of compound 6 a. 


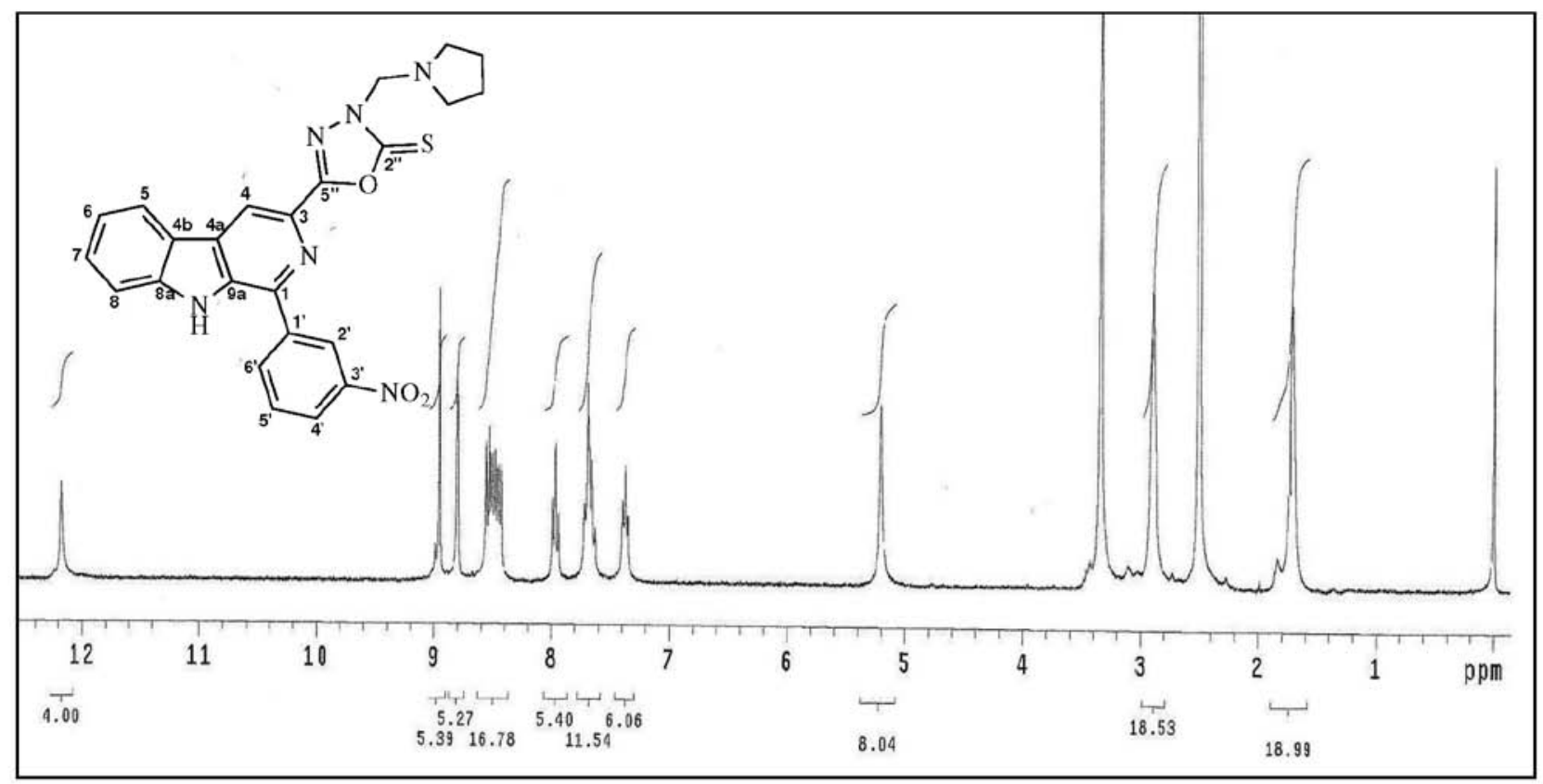

Figure S44. ${ }^{1} \mathrm{H}$ NMR spectra (300 MHz, DMSO- $d_{6}$ ) of compound $\mathbf{6 b}$.

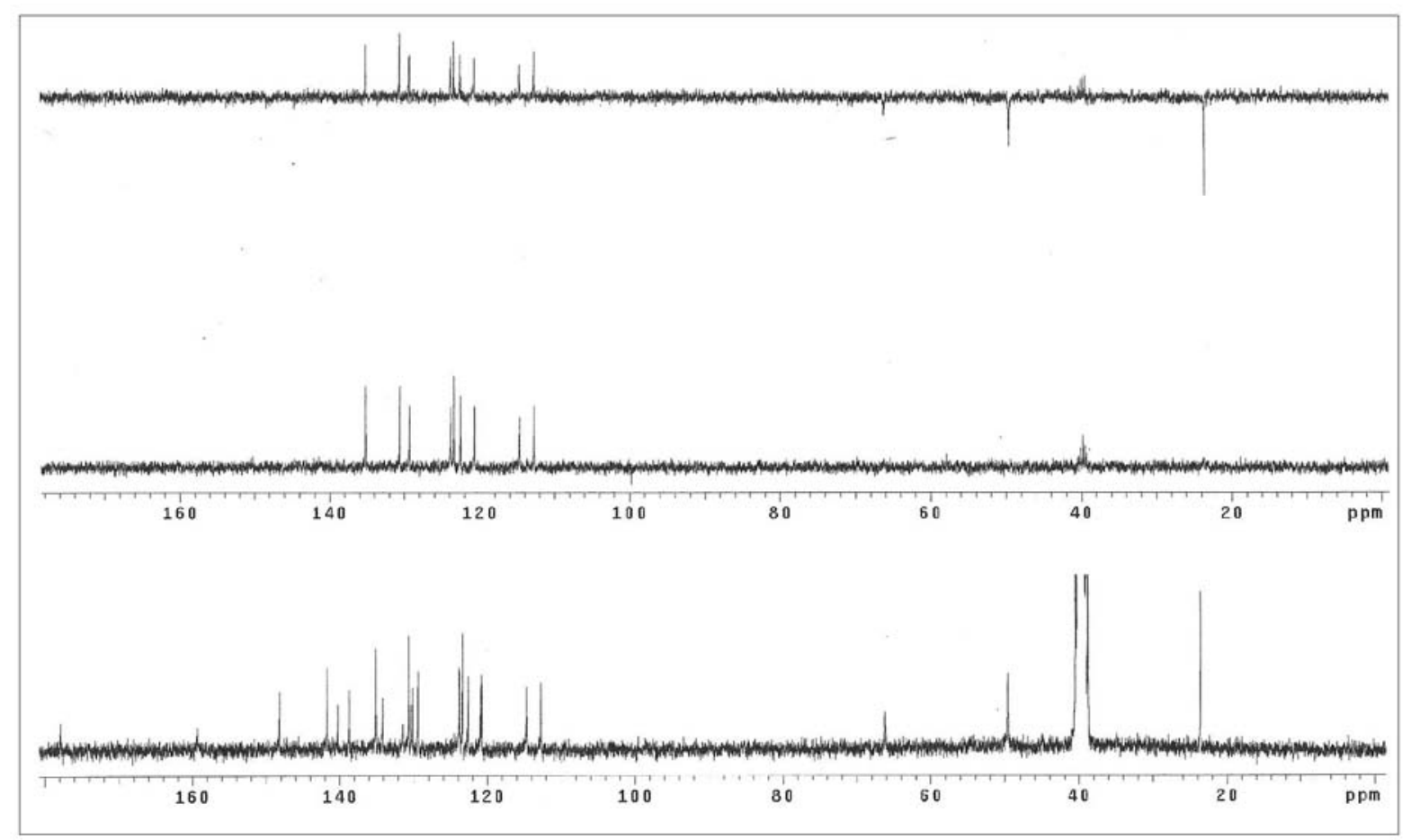

Figure S45. ${ }^{13} \mathrm{C}$ NMR / DEPT spectra (75.5 MHz, DMSO- $d_{6}$ ) of compound $\mathbf{6 b}$. 


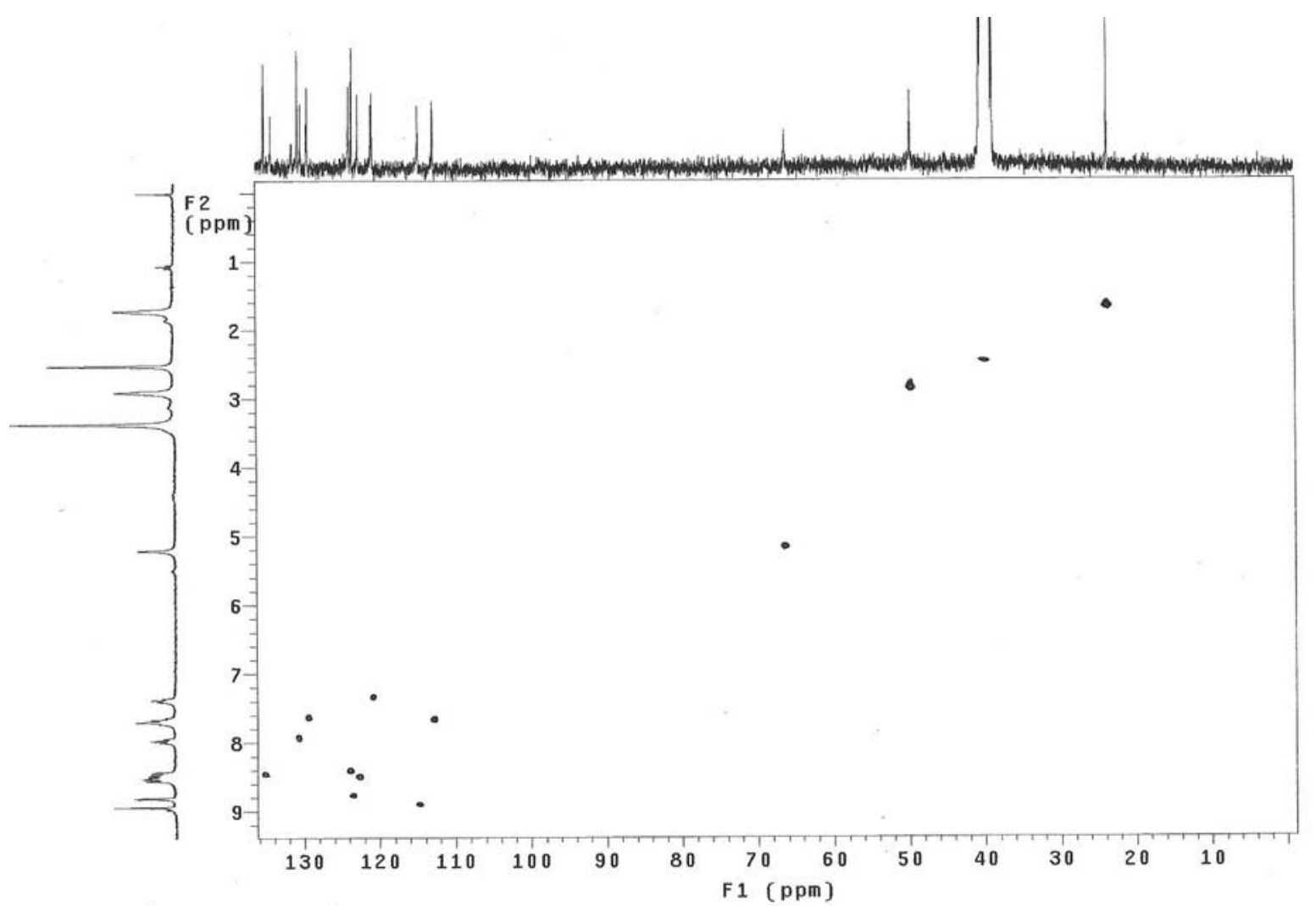

Figure S46. HMQC spectra of compound $6 \mathbf{b}$.

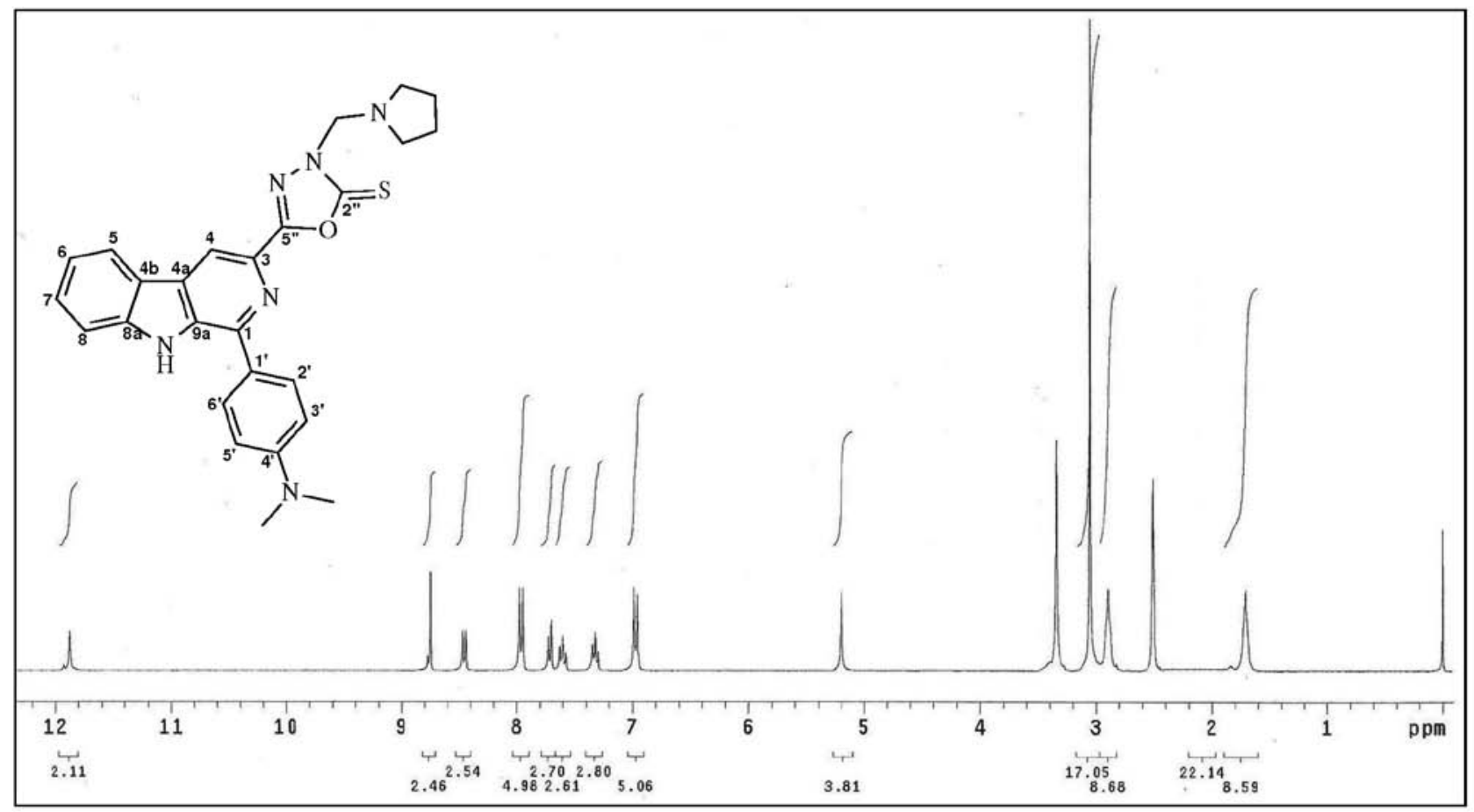

Figure S47. ${ }^{1} \mathrm{H}$ NMR spectra (300 MHz, DMSO- $\left.d_{6}\right)$ of compound $6 \mathbf{c}$. 


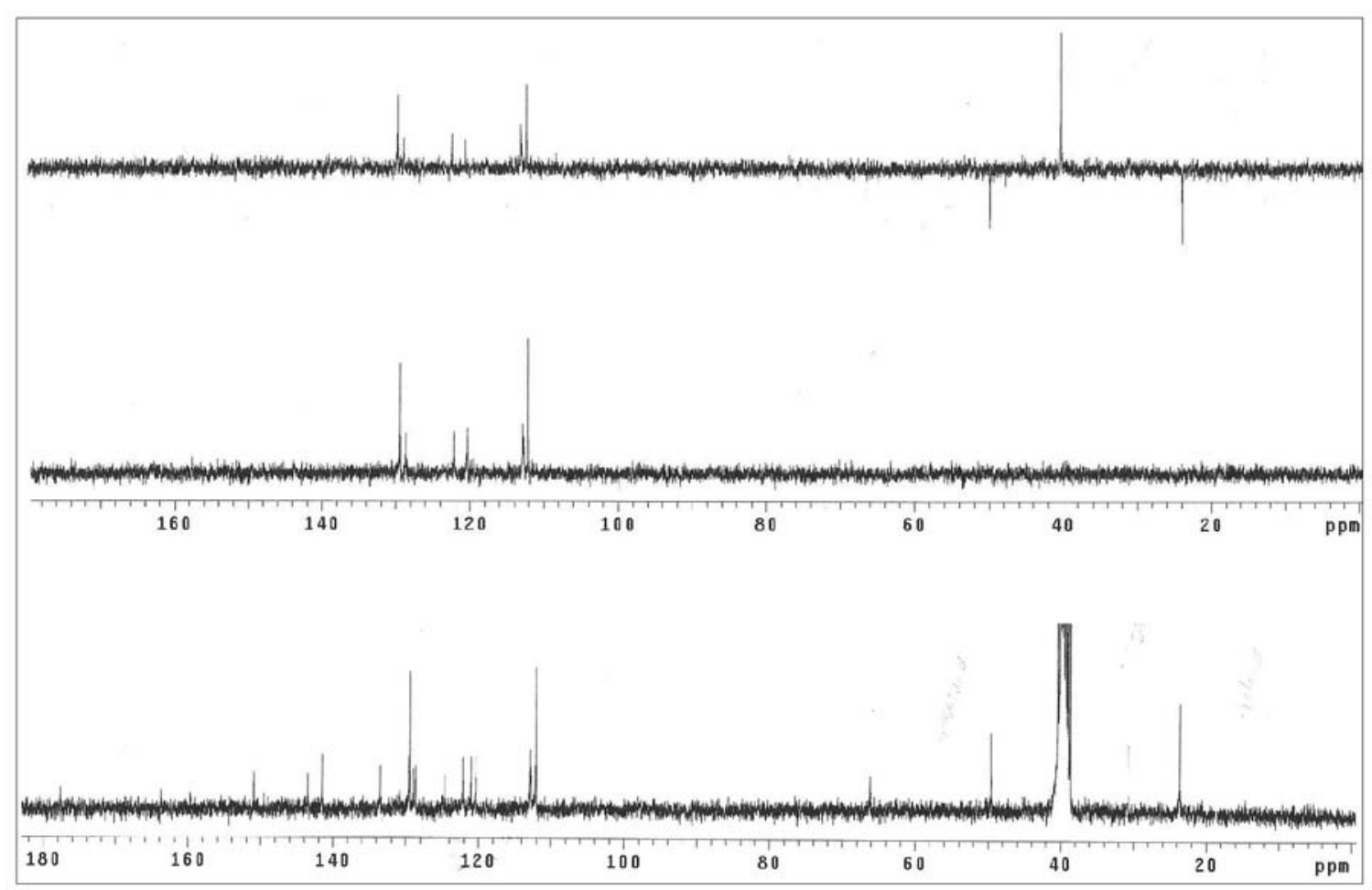

Figure S48. ${ }^{13} \mathrm{C}$ NMR / DEPT spectra (75.5 MHz, DMSO- $d_{6}$ ) of compound $6 \mathbf{c}$.

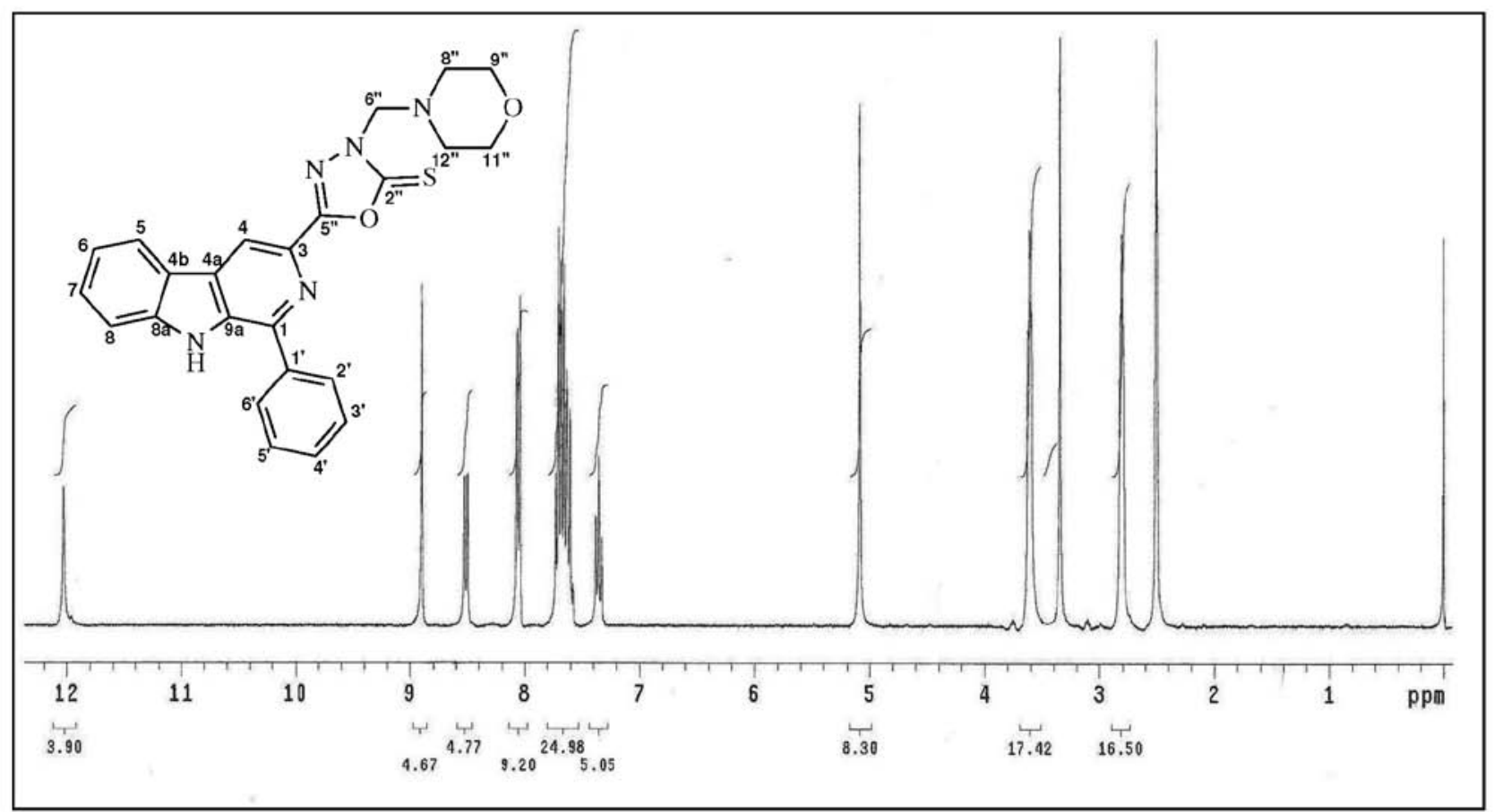

Figure S49. ${ }^{1} \mathrm{H}$ NMR spectra (300 MHz, DMSO- $d_{6}$ ) of compound 7a. 


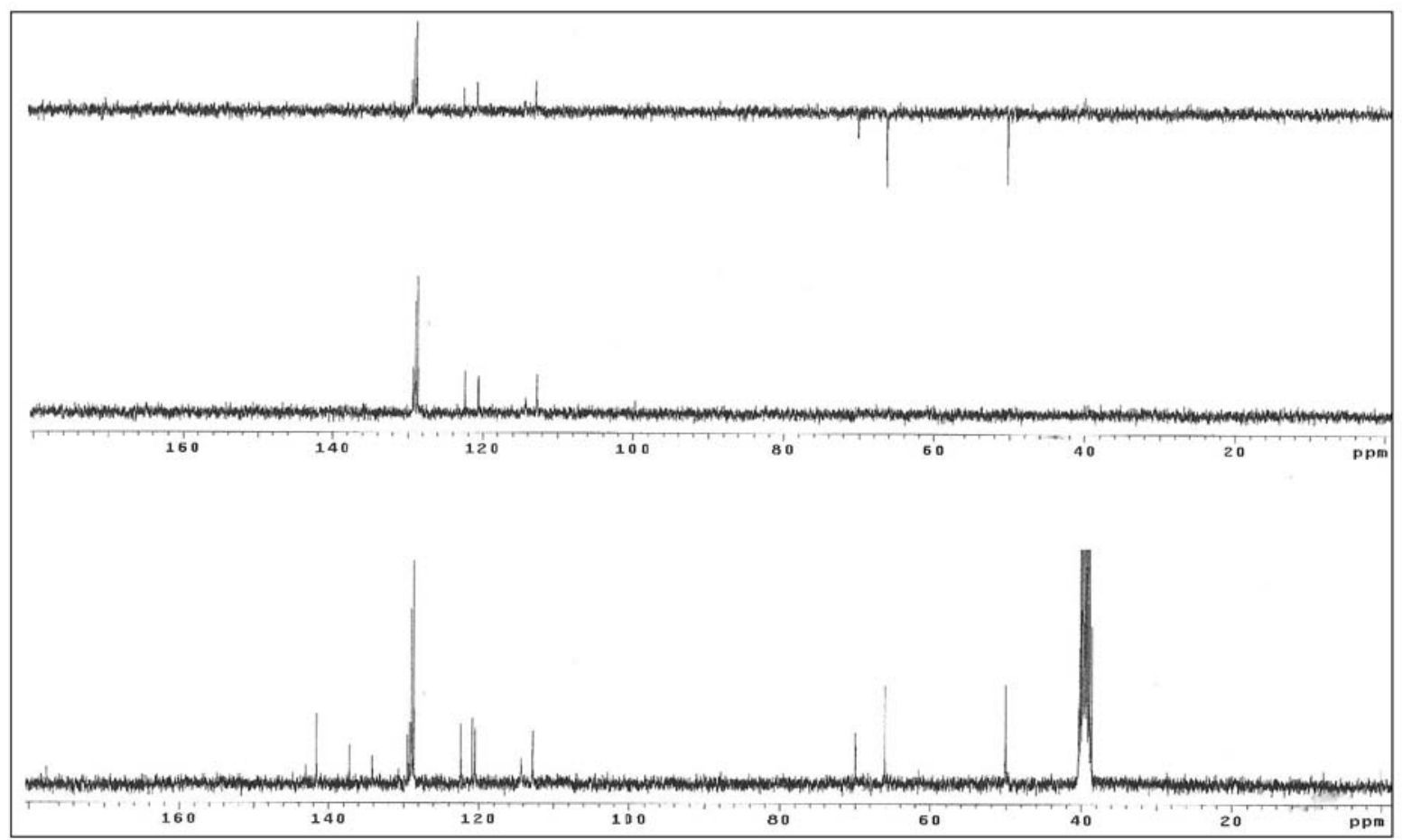

Figure S50. ${ }^{13} \mathrm{C}$ NMR / DEPT spectra (75.5 MHz, DMSO- $d_{\sigma}$ ) of compound 7a.

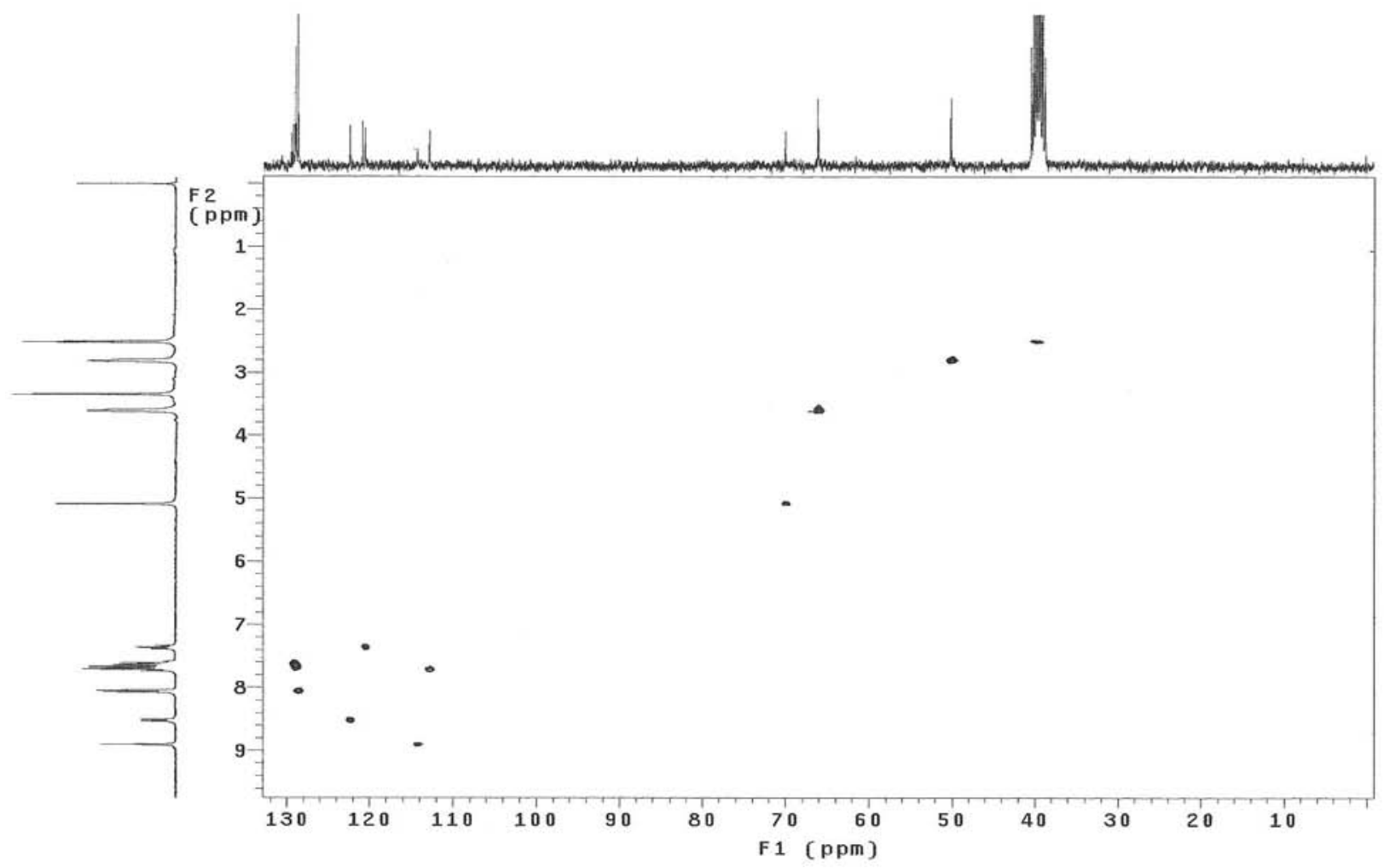

Figure S51. HMQC spectra of compound 7a. 


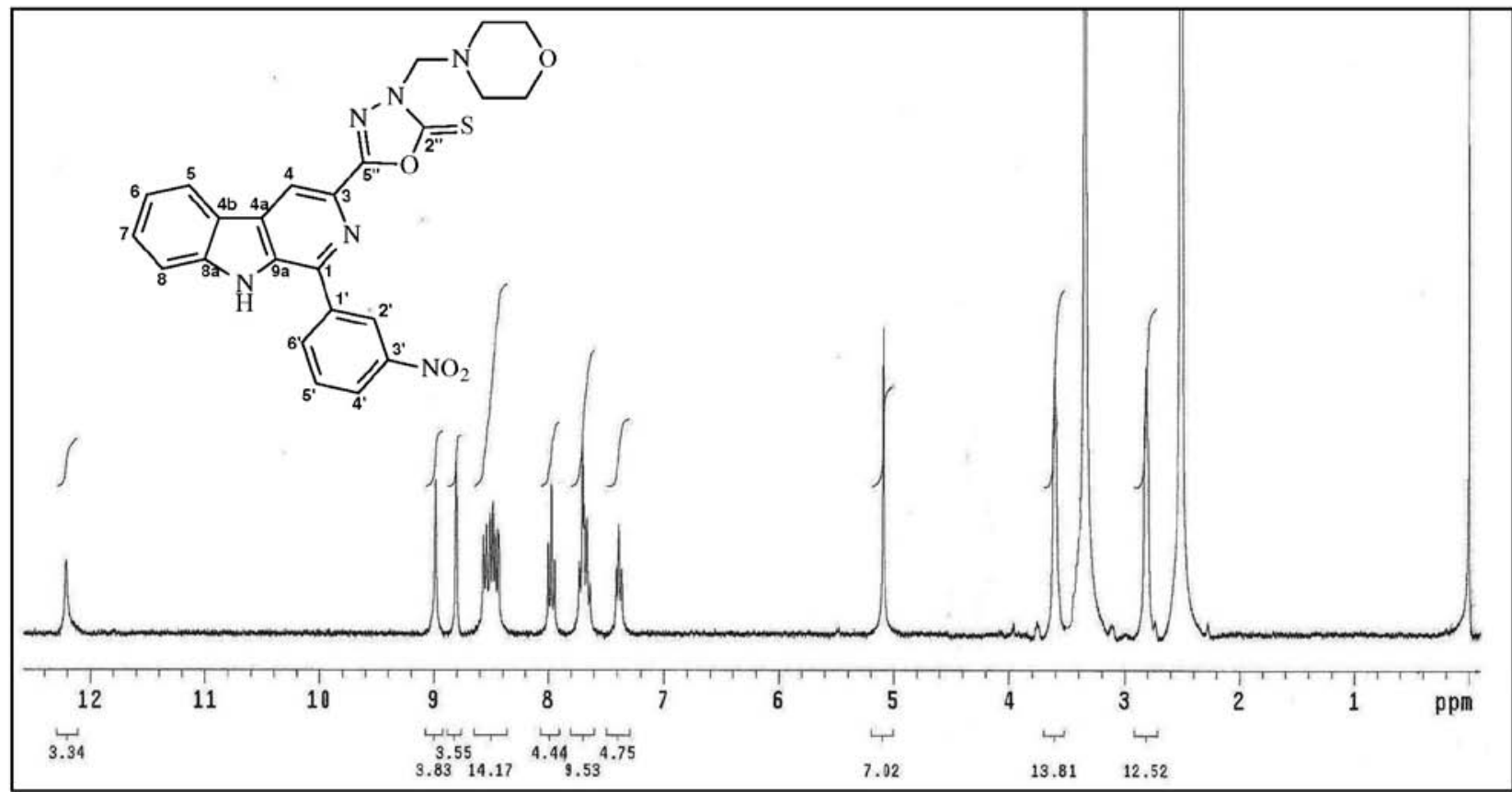

Figure S52. ${ }^{1} \mathrm{H}$ NMR spectra (300 MHz, DMSO- $d_{6}$ ) of compound $7 \mathbf{b}$.

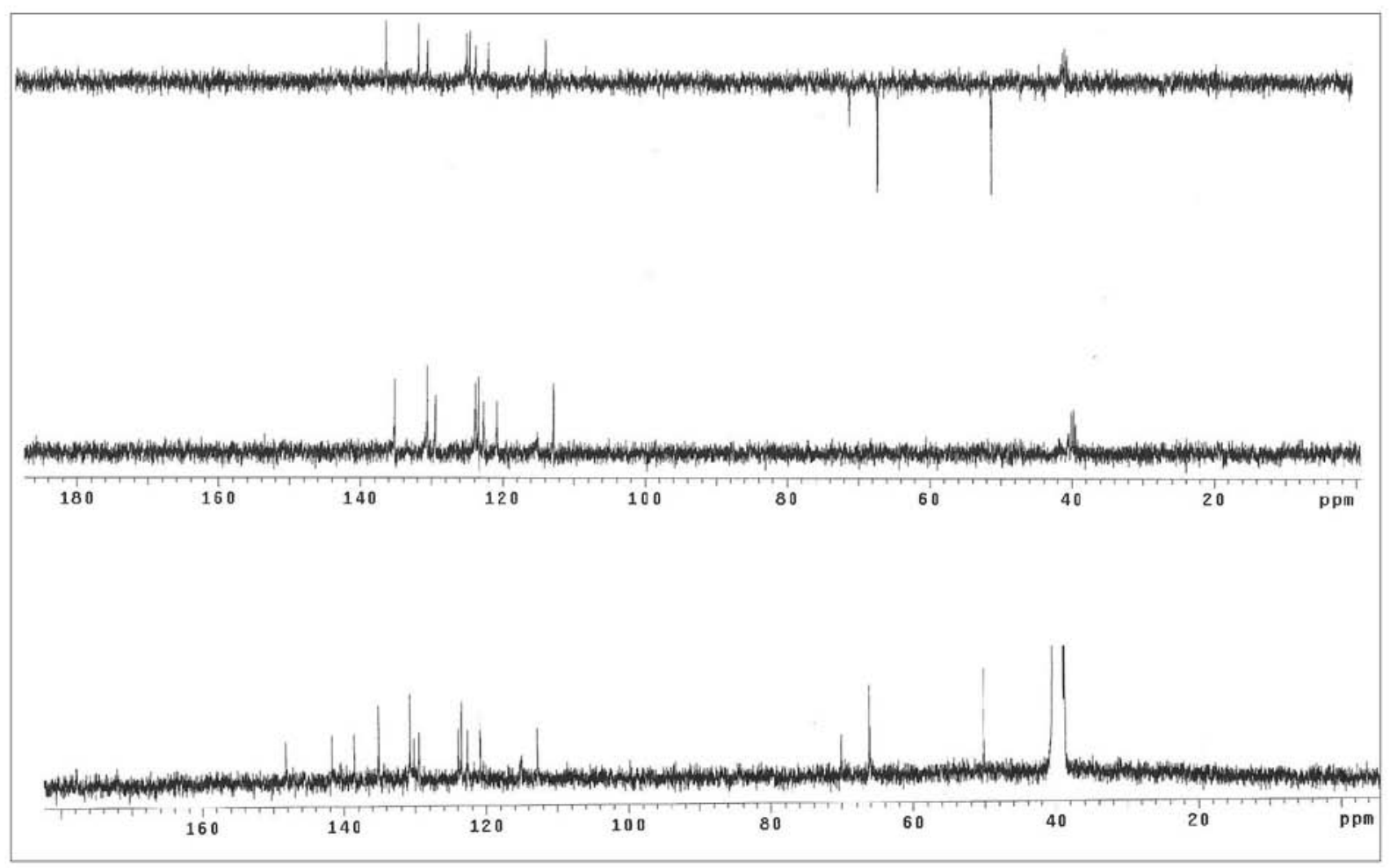

Figure S53. ${ }^{1} \mathrm{H}$ NMR spectra ( $300 \mathrm{MHz}$, DMSO- $d_{6}$ ) of compound $7 \mathbf{b}$. 


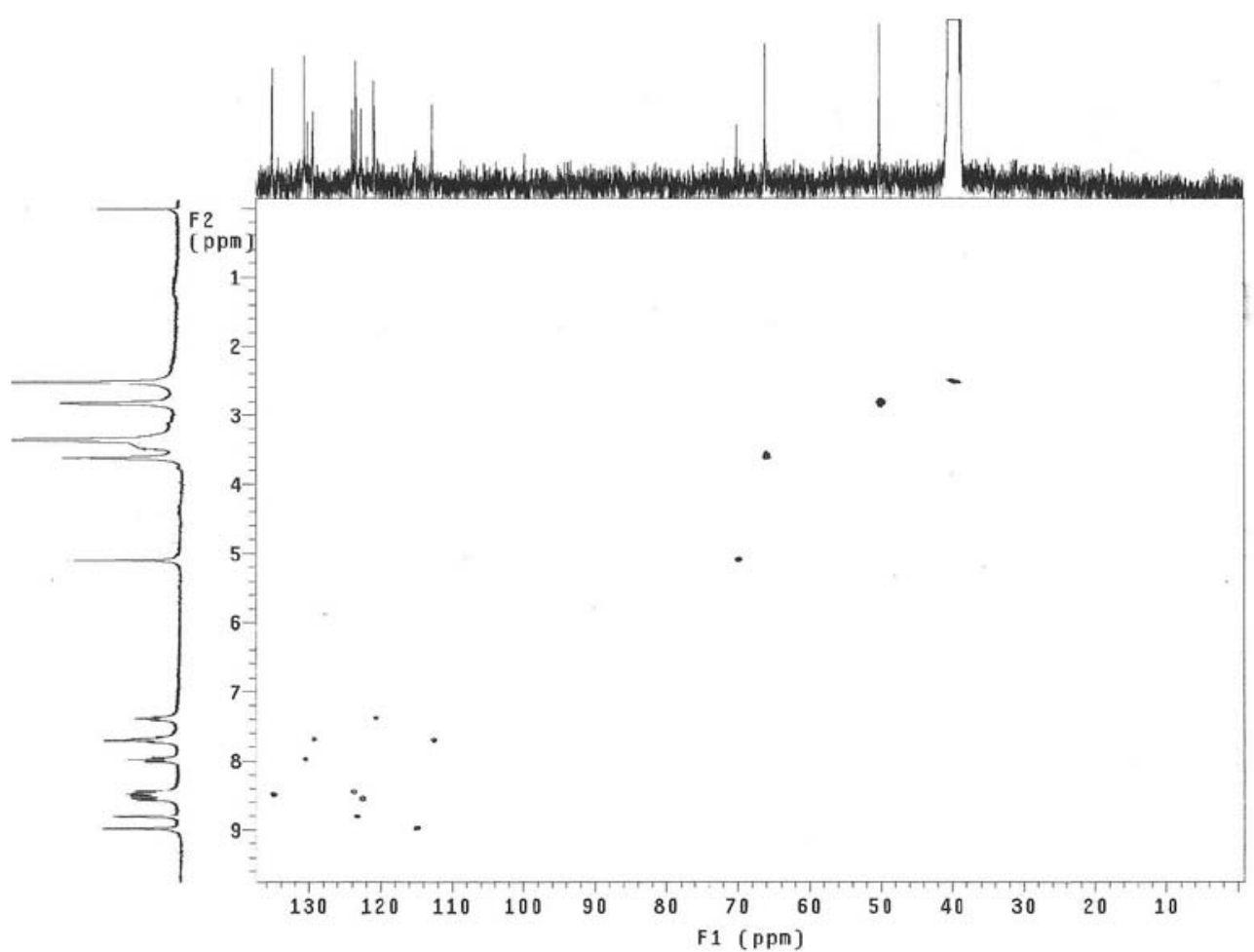

Figure S54. HMQC spectra of compound 7b.

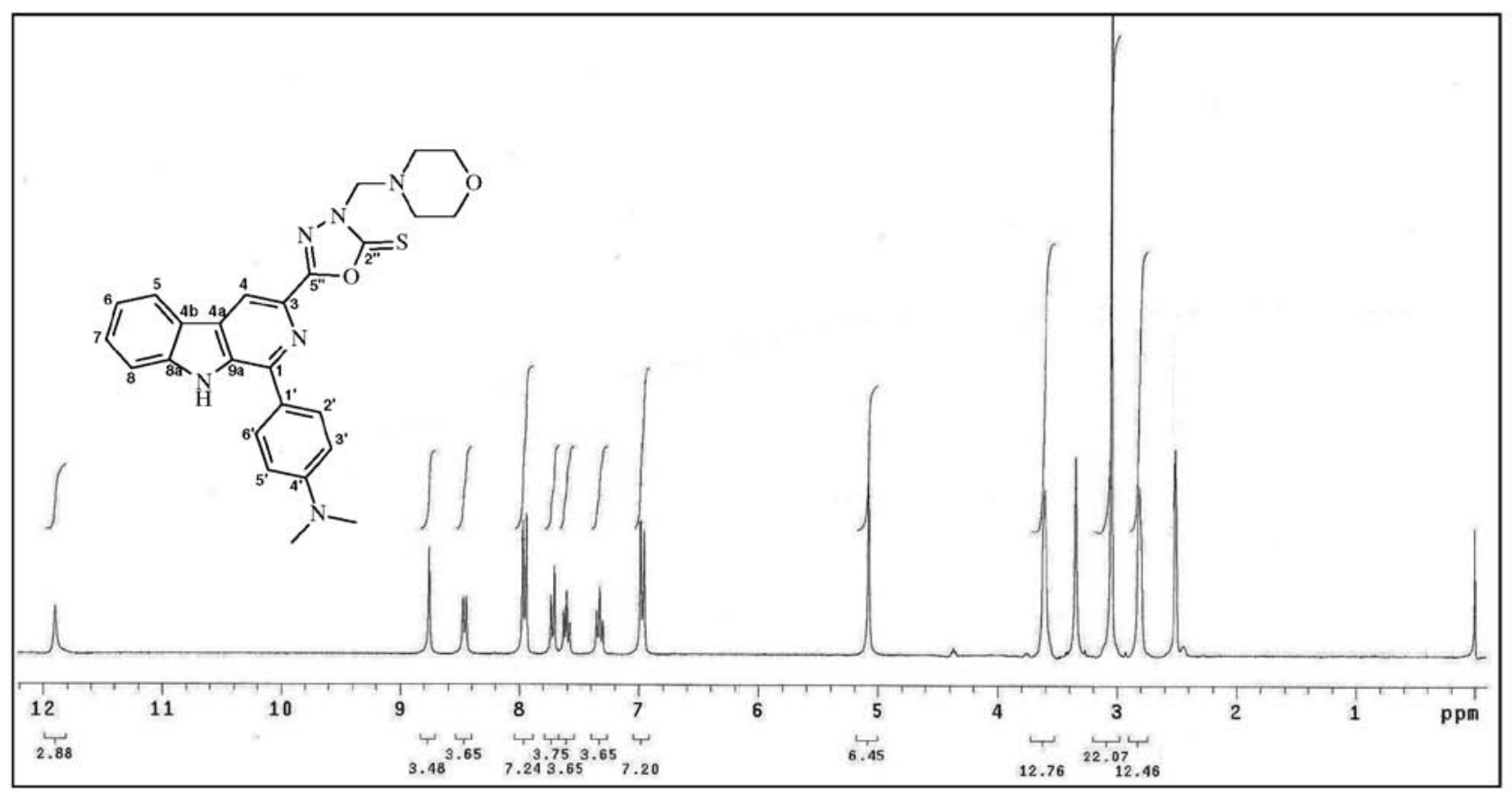

Figure S55. ${ }^{1} \mathrm{H}$ NMR spectra ( $\left.300 \mathrm{MHz}, \mathrm{DMSO}-d_{6}\right)$ of compound 7c. 


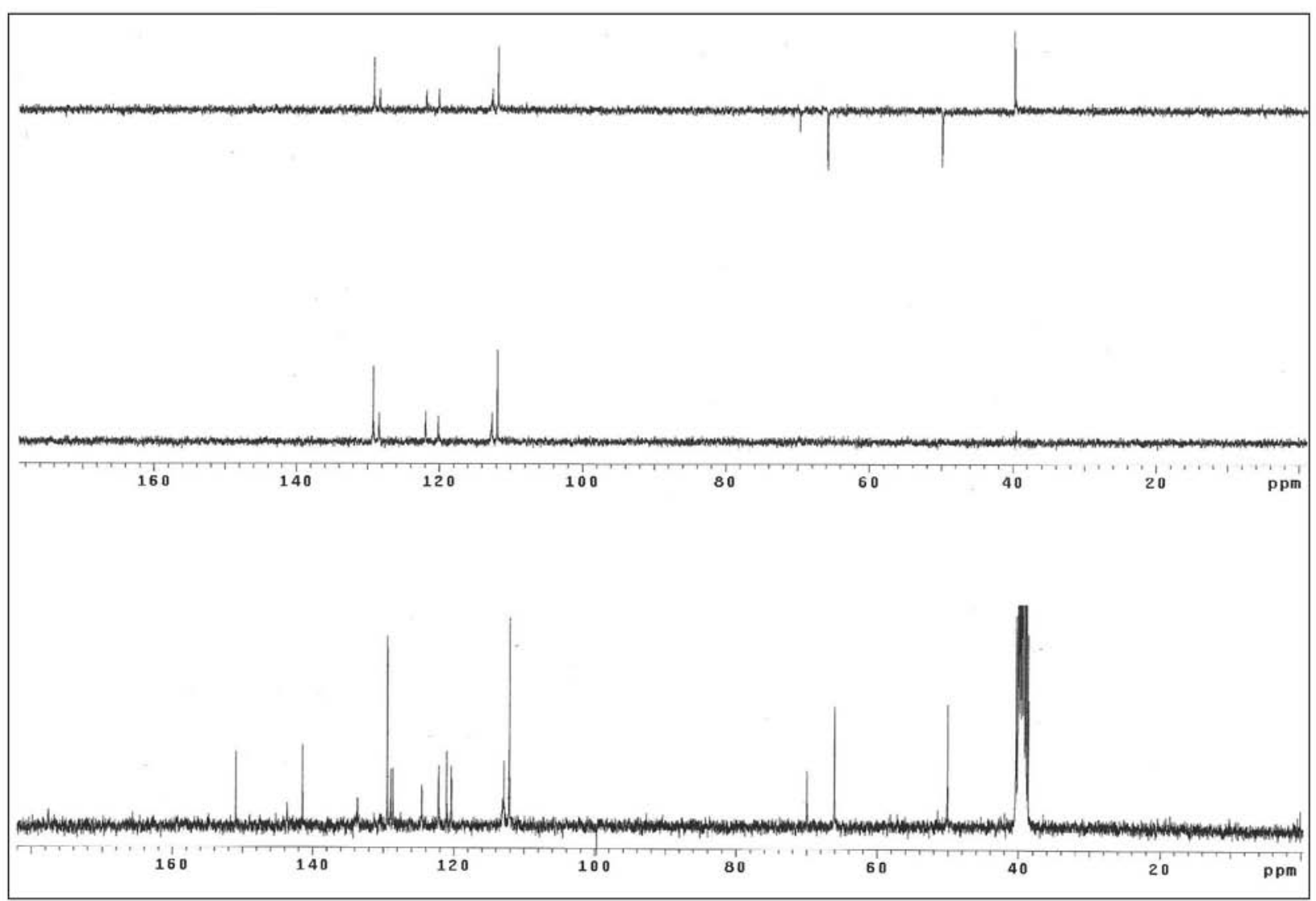

Figure S56. ${ }^{13} \mathrm{C}$ NMR / DEPT spectra (75.5 MHz, DMSO- $\left.d_{6}\right)$ of compound 7c. 